\title{
THE DEVELOPMENT OF AN EMOTION LEXICON FOR THE COFFEE DRINKING EXPERIENCE
}

by

NATNICHA BHUMIRATANA

B.S., University of California, Davis, 2003

M.S., University of California, Davis, 2005

\section{AN ABSTRACT OF A DISSERTATION}

submitted in partial fulfillment of the requirements for the degree

DOCTOR OF PHILOSOPHY

Department of Human Nutrition

College of Human Ecology

KANSAS STATE UNIVERSITY

Manhattan, Kansas 


\begin{abstract}
Coffee is the most consumed beverage after water and the second widely traded commodity after crude oil. In the past decade or so the market for coffee has exploded. It is no longer competitive for the coffee industry to offer consumers just a 'good' cup of coffee. Coffee is mainly consumed for enjoyment and offers consumers the desirable emotional experience. Currently not much work has been done to capture the emotion experiences elicited by coffee drinking, which led to our objective: to identify and assess the feelings that are stimulated by the coffee drinking experience through the development of an emotion lexicon. In the first part of the study, focus groups were utilized to generate and fine-tune a list of emotions that occur during the coffee drinking experience. The list of terms was further refined by check-all-thatapply method, resulting in 86 emotions, which included 39 terms from a recently published emotion lexicon for food. In the second part of the study, this lexicon of 86 emotions was used to evaluate six coffee samples of various origins and degrees of roasting with 94 consumers. The emotion intensities before and during drinking were assessed. Consumers were clustered into six clusters based on the acceptability scores, and the appropriate emotions for distinguishing the coffee samples were determined by Stepwise Regression Analysis, which resulted in 44 emotions. Emotion maps for each consumer cluster constructed using Principal Components Analysis revealed that each sample generated distinctive emotional responses, which varied across each cluster. The last part focused on identifying the sensory drivers of emotions to understand the consumer's perceptions because emotional and sensory experiences determine acceptability and consumption of coffee. Two separate highly-trained panels (a general panel and a coffee panel) performed descriptive analysis of the six coffee samples. After comparing the two panels, the coffee-specific panel data was used to identify the sensory attributes that might be responsible for eliciting certain emotions in the consumers. For instance, darker roasts seem to elicit positive-high energy emotions. Overall, this study is a stepping stone for more in-depth product-specific emotion studies in future.
\end{abstract}




\title{
THE DEVELOPMENT OF AN EMOTION LEXICON FOR THE COFFEE DRINKING EXPERIENCE
}

by

\section{NATNICHA BHUMIRATANA}

B.S., University of California, Davis, 2003

M.S. University of California, Davis, 2005

\begin{abstract}
A DISSERTATION
submitted in partial fulfillment of the requirements for the degree
\end{abstract}

DOCTOR OF PHILOSOPHY

Department of Human Nutrition

College of Human Ecology

\section{KANSAS STATE UNIVERSITY \\ Manhattan, Kansas}

2010

Approved by:

Major Professor

Koushik Adhikari 


\section{Copyright}

NATNICHA BHUMIRATANA

2010 


\begin{abstract}
Coffee is the most consumed beverage after water and the second most widely traded commodity after crude oil. In the past decade or so the market for coffee has exploded. It is no longer competitive for the coffee industry to offer consumers just a 'good' cup of coffee. Coffee is mainly consumed for enjoyment and offers consumers the desirable emotional experience. Currently not much work has been done to capture the emotion experiences elicited by coffee drinking, which led to our objective: to identify and assess the feelings that are stimulated by the coffee drinking experience through the development of an emotion lexicon. In the first part of the study, focus groups were utilized to generate and fine-tune a list of emotions that occur during the coffee drinking experience. The list of terms was further refined by check-all-thatapply method, resulting in 86 emotions, which included 39 terms from a recently published emotion lexicon for food. In the second part of the study, this lexicon of 86 emotions was used to evaluate six coffee samples of various origins and degrees of roasting with 94 consumers. The emotion intensities before and during drinking were assessed. Consumers were clustered into six clusters based on the acceptability scores, and the appropriate emotions for distinguishing the coffee samples were determined by Stepwise Regression Analysis, which resulted in 44 emotions. Emotion maps for each consumer cluster constructed using Principal Components Analysis revealed that each sample generated distinctive emotional responses, which varied across each cluster. The last part focused on identifying the sensory drivers of emotions to understand the consumer's perceptions because emotional and sensory experiences determine acceptability and consumption of coffee. Two separate highly-trained panels (a general panel and a coffee panel) performed descriptive analysis of the six coffee samples. After comparing the two panels, the coffee-specific panel data was used to identify the sensory attributes that might be responsible for eliciting certain emotions in the consumers. For instance, darker roasts seem to elicit positive-high energy emotions. Overall, this study is a stepping stone for more in-depth product-specific emotion studies in future.
\end{abstract}




\section{Table of Contents}

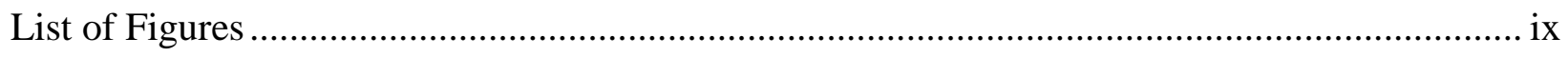

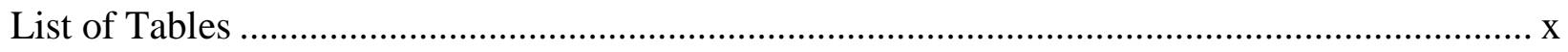

Acknowledgements ............................................................................................................. xi

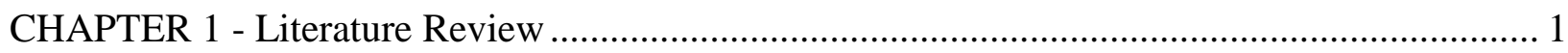

Psychology of Human Emotions ........................................................................................... 1

Defining and Categorizing Human Emotions ...................................................................... 1

Multidimensional and Circumplex models ........................................................................ 1

Prototype approach and hierarchical clustering ............................................................. 4

Emotion Assessment Scales in Clinical Psychiatry Practice ................................................... 5

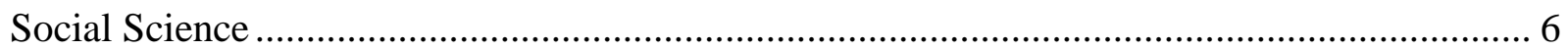

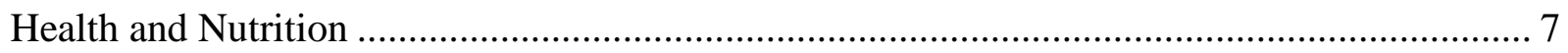

Assessment Scale for Health and Food Choice ……….......................................................... 7

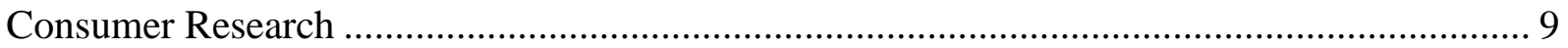

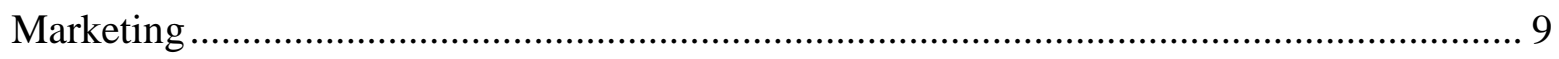

Consumption Experience ……………………………………………………………... 10

The Consumption Emotions Set (CES) ……………................................................ 10

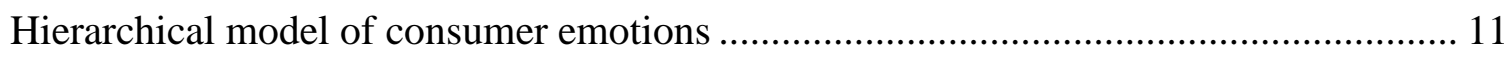

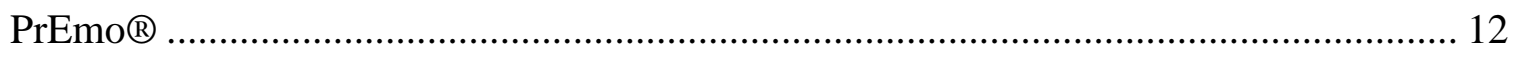

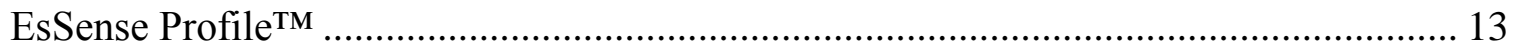

Geneva Emotion and Odor Scale (GEOS) …………................................................ 16

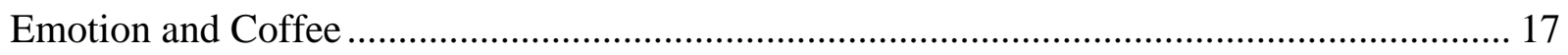

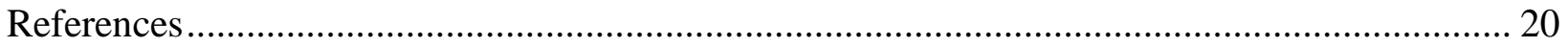

CHAPTER 2 - The Development of Emotion Lexicon for Coffee Drinking Experience ……..... 25

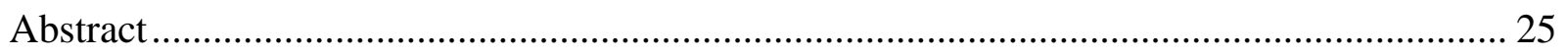

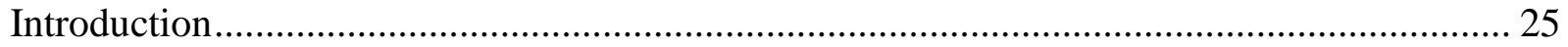

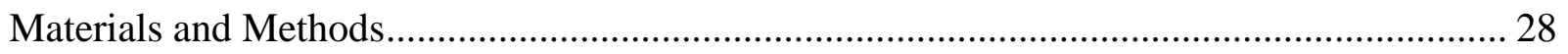

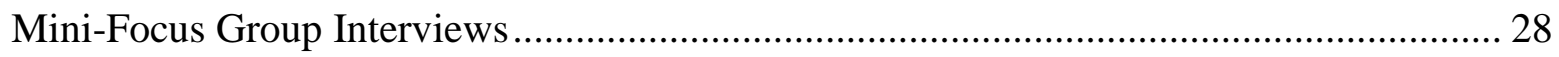

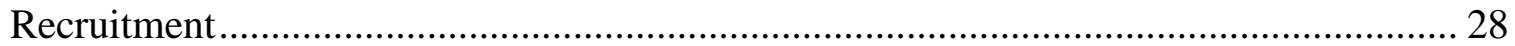




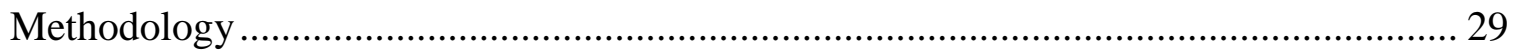

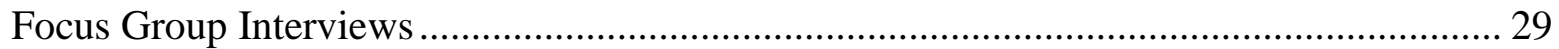

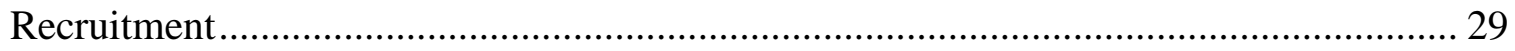

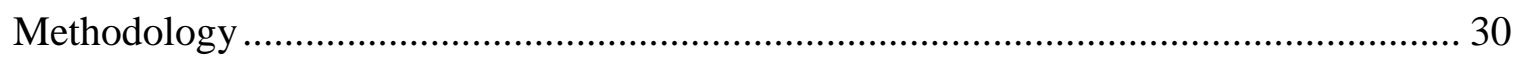

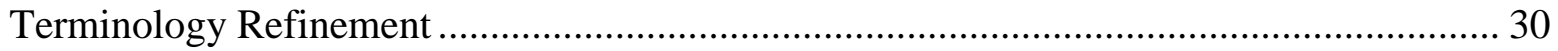

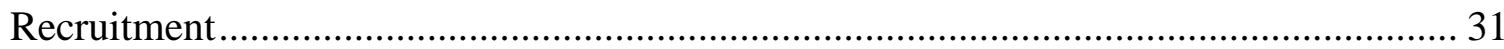

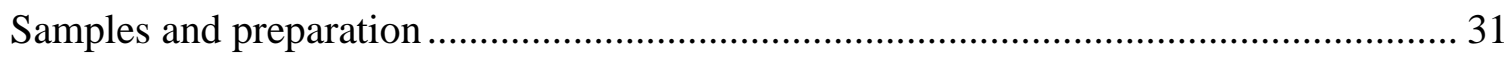

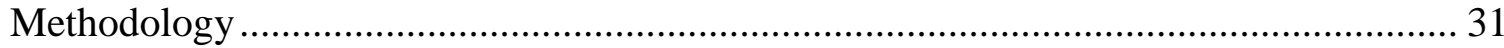

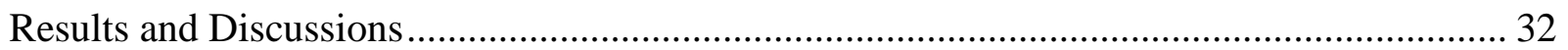

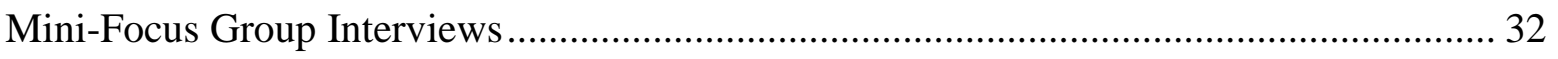

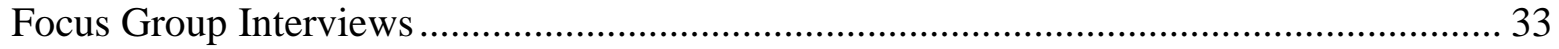

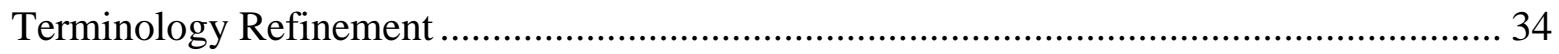

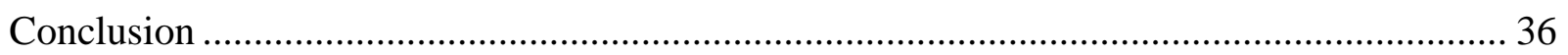

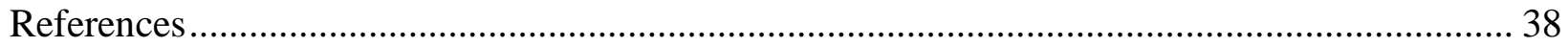

CHAPTER 3 - Examining the "coffee drinking experience" emotion lexicon .......................... 41

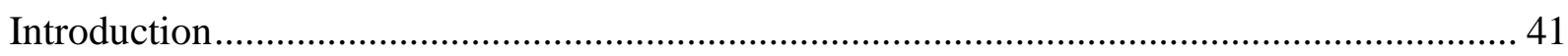

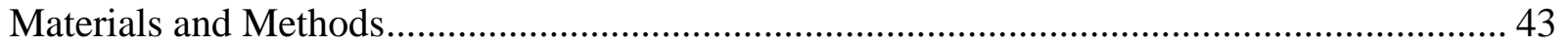

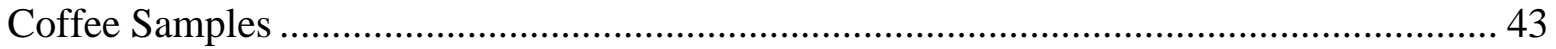

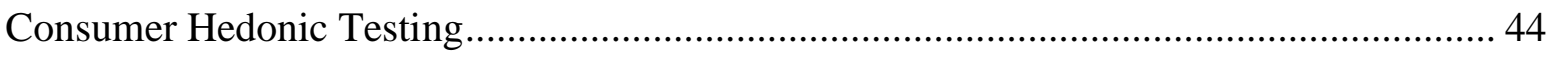

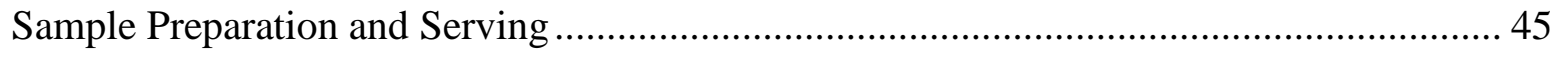

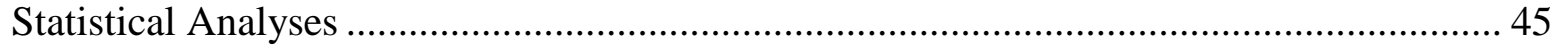

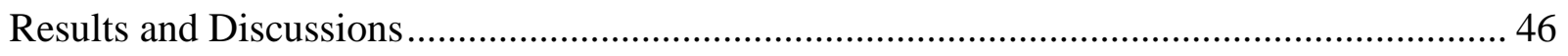

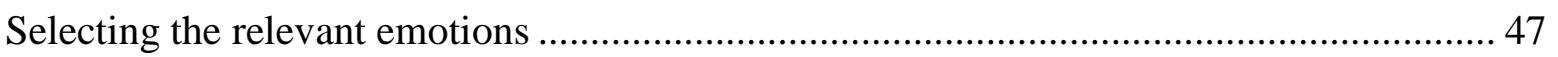

Emotion Profiles for Clusters of Coffee Users ............................................................ 50

Consumer cluster 1: users who like all coffee samples ......................................... 50

Consumer cluster 2: users who disliked Breakfast ................................................. 51

Consumer cluster 3: users who liked Breakfast and Nantucket.................................. 53

Consumer cluster 4: users who did not give high scores to any coffee sample ............... 54

Consumer cluster 5: users who liked Breakfast and disliked the rest........................... 55

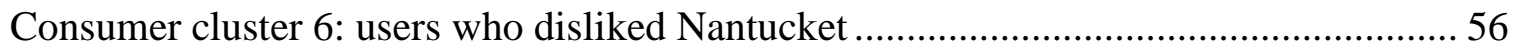

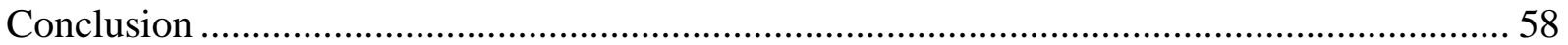


CHAPTER 4 - Coffee Drinking and Emotions: Are There Key Sensory Drivers for Emotions? 62

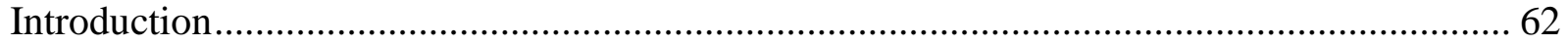

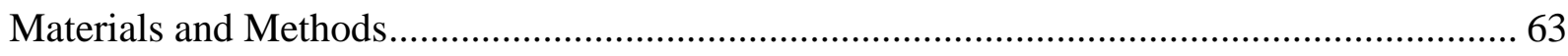

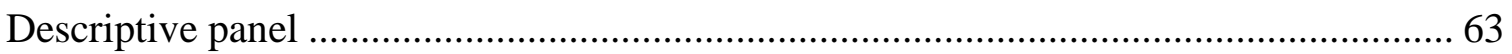

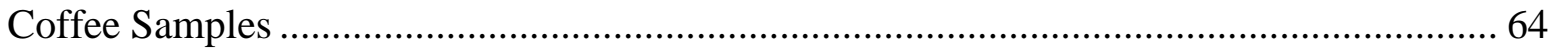

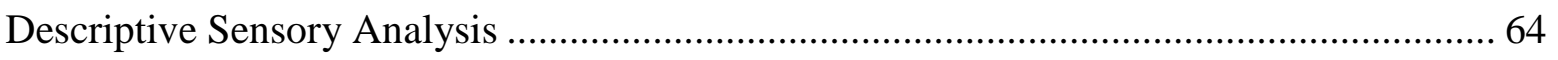

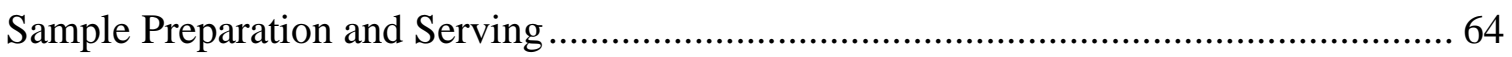

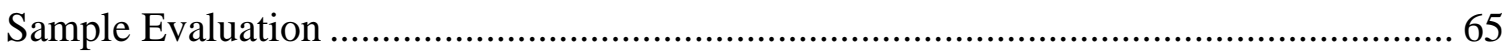

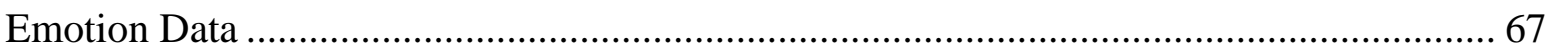

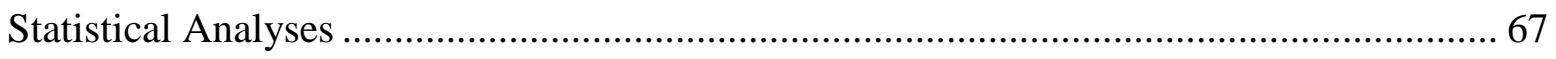

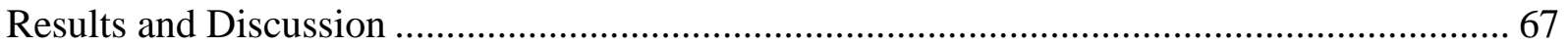

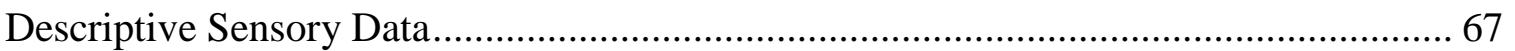

Identifying Sensory Drivers for the Emotional Experience..................................... 70

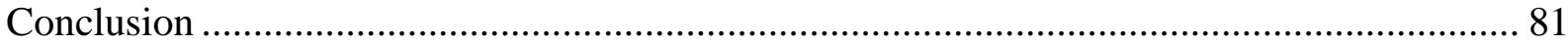

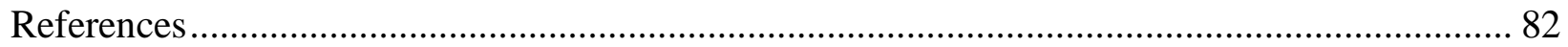

CHAPTER 5 - Conclusions and Future Research ............................................................ 85

Appendix A - Moderator's guide for mini-focus group interview ......................................... 87

Appendix B - Moderator's guide for focus group interview .............................................. 91

Appendix C - Questionnaire for the consumer test...................................................... 92

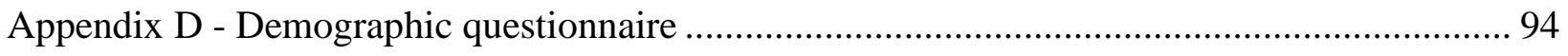

Appendix E - Emotion terms resulted from Stepwise analyses: all consumers......................... 95

Appendix F - Emotion Terms resulted from Stepwise analyses: by consumer clusters .............. 96

Appendix G - Emotion terms resulted from Stepwise Analyses: by product clusters ................. 99 


\section{List of Figures}

Figure 1.1. The Consumption Emotion Set (CES; Richins, 1997) ......................................... 11

Figure 1.2. Hierarchical model of consumer emotions (Laros \& Steenkamp, 2005) ................. 12

Figure 1.3. EsSense Profile ${ }^{\mathrm{TM}}$ ballot for acceptability and emotion intensities (King \&

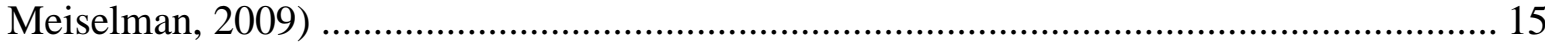

Figure 1.4. Six dimensions that describe affective feelings elicited by odors (GEOS; Chrea et al.,

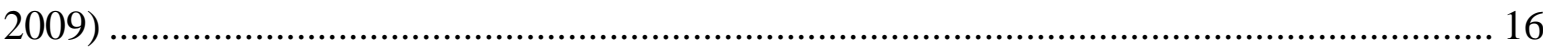

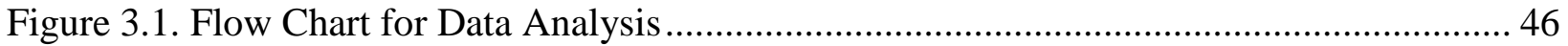

Figure 3.2. Coffee Drinking Experience (CDE) emotion profile for Cluster 1........................ 51

Figure 3.3. Coffee Drinking Experience (CDE) emotion profile for Cluster 2 ....................... 52

Figure 3.4. Coffee Drinking Experience (CDE) emotion profile for Cluster 3 ........................ 54

Figure 3.5. Coffee Drinking Experience (CDE) emotion profile for Cluster 4........................ 55

Figure 3.6. Coffee Drinking Experience (CDE) emotion profiles for Cluster 5 ....................... 56

Figure 3.7. Coffee Drinking Experience (CDE) emotion profile for Cluster 6....................... 57

Figure 4.1. Sensory profiles of the six coffees generated by the coffee panel. ....................... 69

Figure 4.3. Partial Least Square Regression Analysis of Descriptive Sensory and Emotion Data

of the Six Coffee Samples for 94 Consumers ........................................................... 72

Figure 4.4. Partial Least Square Regression Analysis of Descriptive Sensory and Emotion Data of the Six Coffee Samples for Consumer Cluster 1 .................................................... 75

Figure 4.5. Partial Least Square Regression Analysis of Descriptive Sensory and Emotion Data of the Six Coffee Samples for Consumer Cluster 2 ..................................................... 76

Figure 4.6. Partial Least Square Regression Analysis of Descriptive Sensory and Emotion Data of the Six Coffee Samples for Consumer Cluster 3 ................................................. 77

Figure 4.7. Partial Least Square Regression Analysis of Descriptive Sensory and Emotion Data

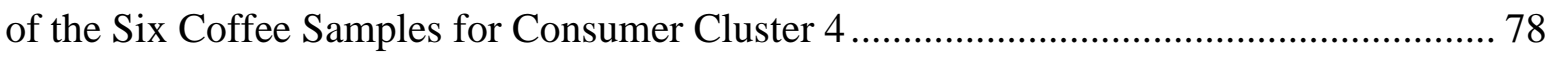

Figure 4.8. Partial Least Square Regression Analysis of Descriptive Sensory and Emotion Data of the Six Coffee Samples for Consumer Cluster 5 ....................................................... 79

Figure 4.9. Partial Least Square Regression Analysis of Descriptive Sensory and Emotion Data

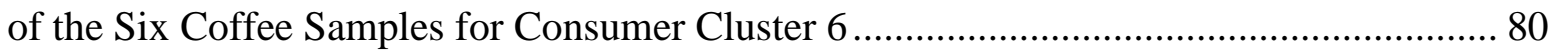




\section{List of Tables}

Table 2.1. Emotion terms generated by mini-focus group discussions ..................................... 32

Table 2.2. List of emotion terms fine-tuned by the focus group discussions ........................... 33

Table 2.3. Final list of emotion term refined by the check-all-that-apply method ...................... 35

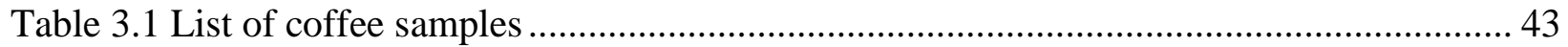

Table 3.2. Average liking scores for each consumer cluster and coffee sample ..................... 47

Table 3.3. Lists of emotions derived from the coffee drinking experiences (CDE) .................. 49

Table 4.1. The list of aroma, flavor, and texture descriptors identified by the coffee panel ........ 66

Table 4.2. The sensory attributes detected by the coffee panel to significantly differentiate the six

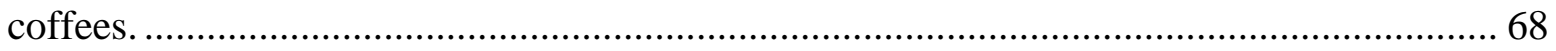




\section{Acknowledgements}

I would like to thank my major advisor Dr. Koushik Adhikari for giving me the opportunity to work under his supervision and for his mentorship, encouragement and support throughout my Ph.D. program. I am deeply thankful for all the thoughtful little things he did to make my stressful times easier to cope with and for always having my back. I would also like to thank my committee members for their guidance and constructive comments, particularly, Dr. Edgar Chambers, IV and Dr. Leigh Murray. Their knowledge, expertise and advice led me to the accomplishment of my Ph.D. study and were very much appreciated.

I would like to thank the Wolf Group for sponsoring part of my research, especially Mona Wolf, who has been very kind and generous. I will always be grateful for her hospitality and support. Also, I thank the Sensory Analysis Center for the hands-on sensory training and practical knowledge beyond the textbooks. Thank you for keeping me on my toes at all times and for preparing me for the corporate culture.

Thanks to all my friends who I met during my time at Kansas State University, especially my roommate, Erika Bono, and my officemate, Aussama Soontrunnarudrungsri. Thank you for listening to my endless flow of thoughts (i.e. complaints) and for being my accomplices when I needed to satisfy my urge to shop, eat, drink, and dance. They made my life more bearable and enjoyable. I would also like to express my gratitude towards Jeehyun Lee for the motivations and research ideas. She has always been very caring and will continue to be my inspiration to be better in the field of Sensory Analysis. I would like to thank Laura Vazquez-Aruojo for her sincerity and friendship. Her willingness to help me in any way she could and her witty personality made the last stretch of my $\mathrm{PhD}$ program more entertaining and much less stressful.

My deep appreciation goes out to my family who has always pushed me in every way and gave me the support I needed to achieve my Ph.D. degree. Without their encouragement I would not have pursued and gone through with the Ph.D. study in the first place. I would like to gratefully acknowledge Watcharapol Chayaprasert for being my role model both personally and professionally. He has set the standards high and consistently exceeds his own standards in all aspects of life, which was very motivating. I thank him for all the inspirations and encouragement to get me through every difficult situation and for being a strong shoulder I know I could lean on when things get rough. 


\section{CHAPTER 1 - Literature Review}

Emotions have been researched extensively across many disciplines, including psychology, social science, health and nutrition, and consumer research. Researchers continuously disagree on the definitions and categorization of emotions. Therefore, many theories have been developed with an attempt to define the emotion and refine the terms it entails. Mood and emotion scales have been constructed to measure and evaluate the state of human psychology when experiencing stimuli.

\section{Psychology of Human Emotions}

\section{Defining and Categorizing Human Emotions}

In an attempt to explain the emotion episodes and overall nature of human emotions, researchers proposed several categorizing systems or models to organize human emotions.

\section{Multidimensional and Circumplex models}

Over the years, researchers proposed dimensionality in human emotions, with positivenegative valence, activity/arousal, level of engagement, and potency/dominance being the most frequently obtained dimensions. Thayer (1989) developed a multidimensional test of different arousal emotion states called "The Activation - Deactivation Adjective Check List" (AD ACL). Two dimensions of arousal emotions were measured, and subsequently referred to as energetic arousal and tense arousal. In contrast to the basic emotions, these emotions are bipolar; each emotion term represents a continuity of mood state, with the two opposite emotion adjectives anchoring at each end of the spectrum. Energetic arousal dimension contrasts vigor and energy with tiredness and fatigue, whereas, tense arousal dimension contrasts tension and nervousness with relaxation and calmness. These two arousal dimensions reflect the activity of human evolution-biopsychological systems, where energetic arousal is the human response to vigorous action, and tense arousal is the human reflex to danger, as well as, inhibiting activities in order to maintain energy for future threats (Thayer, 1967, 1978). Izard $(1977,2009)$ believed emotion is derived from evolution and neurobiological development. He proposed a differential emotion theory (DET) where emotions can be divided into two categories - basic emotions and emotion schemas. Izard defined basic emotions as those fundamental to human mentality assisting and 
stimulating adaptive responses vital for survival and well-being. These emotions are generated inevitably by the brain upon sensing environmental stimuli. Emotion schema is a process involved in the dynamic interaction of basic emotions and cognitions that create perceptions and thoughts. These emotional experiences result from emotion schemas consisting of the same basic emotion state, but more complicated due to thought processes and perceptual biases. Also, using the psycho-evolutionary theory, Plutchik $(1980,2001)$ defined emotions in terms of their adaptive functions that enable animals (humans) to cope with the crises in life, included hierarchy, territoriality, identity, and temporality. He indicated that emotions are biologically primitive and have evolved to increase the survival chance of an animal. He developed a wheel of emotions to describe the relationships and interconnections between each emotion. The wheel of emotions is comprised of eight primary bipolar emotions: anger - fear, joy - sadness, trust disgust, and surprise - anticipation. Taking the idea from the color wheel, Plutchik presented a circumplex model suggesting that primary emotions can be expressed at different intensities as well as can be mixed with one another to form different emotion.

Also in 1980, Russell and Pratt described that cognitive behavior and affective behavior define the interaction between a person and the environment. Emotions are the affective behavior. They proposed that a person's affective feelings are categorized into a twodimensional bipolar space that can be defined by 8 emotion variables. They considered pleasure, arousal, excitement, and stress to be continuous dimensions. Pleasure is the bipolar opposite of displeasure; arousal is the bipolar opposite of sleepiness; excitement is the bipolar opposite of depression; and stress is the bipolar opposite of relaxation. They indicated that pleasure displeasure axis is orthogonal to arousal - sleepiness axis, and stress - relaxation axis is orthogonal to excitement - depression axis. The relationships among the 8 emotion variables were described in an affect model where each falls $45^{\circ}$ from each other in a circular order: pleasure $\left(0^{\circ}\right)$, excitement $\left(45^{\circ}\right)$, arousal $\left(90^{\circ}\right)$, distress $\left(135^{\circ}\right)$, displeasure $\left(180^{\circ}\right)$, depression $\left(225^{\circ}\right)$, sleepiness $\left(270^{\circ}\right)$, and relaxation $\left(315^{\circ}\right)$. Pleasure and arousal were indicated to be the main dimensions of emotions experienced in human. Russell (1980) then validated this theory by having subjects scaling and categorizing 28 emotion adjectives in four different ways and later an affect grid was developed to assess human emotion along the dimensions of pleasure displeasure and arousal - sleepiness (Russell, 1989). 
Similarly Watson and Tellegen (1985) attempted to uncover a range of emotions and also revealed the two-factor model for emotions. As a result of factor analysis of the self-rated mood, they observed that there exist two dominant mood dimensions: Positive Affect and Negative Affect. They proposed a bipolar structure emotion model with high positive - low positive (Positive Affect) and high negative - low negative (Negative Affect) as the main dimensions which were orthogonal to each other. They further clarified that there two dimensions by no means categorize all of the human emotions. Positive Affect and Negative Affect factors merely accounted for $50-75 \%$ of the variance. Other factors involved are pleasantness and level of engagement. Many emotion items cannot be identified as pure positive or negative affect but a mixture of the two. The pleasantness and engagement factors represents terms that are a fusion of high positive affect and low negative affect or any other combination of the two. Based on this finding, Watson, Clark and Tellegen (1988) took this information a step further and developed the Positive Affect - Negative Affect Schedule (PANAS) to assess a person's feelings during a certain time frame. The scale consists of 160 adjectives, which was proven to be too lengthy to provide consistent validated results. To resolve this problem, Watson et al. (1988) developed a brief version of PANAS by selecting 10 emotion items to represent each of the positive and negative effects. They verified that the scale provided consistent results and is able to detect the emotional fluctuation of different individuals. Emotion terms are rated on a 5-point scale anchored by "not at all to very slightly" (1) to "extremely" (5). In 2004, Crawford and Henry validated the PANAS with a non-clinical sample of 1,003 adults and supported PANAS's reliability and validity.

Adding to the previous theories, Mayer and Gaschke (1999) suggested another dimension of emotion by indicating that mood may be experienced on two levels: direct and reflective. Emotions that are experienced along the pleasant - unpleasant and arousal - calm magnitudes are at the direct level. These moods include basic emotions, as well as cognitive emotions. A reflective level of mood occurs as an effect of the direct perception or judgment of mood; these moods can potentially modulate the overall mood. The researchers referred to the reflective experience of mood as 'meta-mood experience' (1999). Two scales were subsequently developed: MIS (Mood-State Introspection Scale) and BMIS (Brief Mood-State Introspection Scale). BMIS is the brief version of MIS when taking brevity into consideration. It contains two emotion adjectives representing the determined eight mood states (happy, loving, calm, 
energetic, fearful/anxious, angry, tired, and sad), resulting in a total of 16 terms. A 4-point scale (anchored by 'definitely do not feel' (1), 'do not feel' (2), 'slightly feel' (3), 'definitely feel' (4)) is used to measure each item. The scale instructs the individual to circle the extent in which the adjective indicates one's present mood. MIS is a 62-term adjective check-list covering 10 mood states. Eight of these mood states are present in the BMIS, but each consists of seven mood items on MIS. The two mood states that are not included in BMIS are boredom and pride, because these two categories represented a loose group of emotion terms.

\section{Prototype approach and hierarchical clustering}

Other researchers took a slightly different approach and attempted to categorize emotions into structural models. Rosch $(1973,1978)$ proposed the prototype theory where emotions are mapped out in vertical and horizontal dimensions. The vertical dimension explains the hierarchical relations among emotions in a tree-like structure of the domain of emotion concepts, including 3 levels: superordinate, basic, and subordinate. The basic-level emotions are assessed most quickly and frequently in everyday emotion episodes and are distinctive among each category. Horizontal dimension groups similar emotions and demonstrates the vague boundaries between each emotion within the same basic-level emotion. Shaver, Shwatz, Kirson, and O'Connor (1987) explored and further validated Rosch's Prototype theory. They examined the hierarchical structure of the emotion domain and compared it with the circumplex and multidimensional structures by using factor analysis and multidimensional scaling techniques. The resulted clusters of basic emotions were similar to other theorists' findings: love, joy, anger, sadness, and fear. Shaver et al. pointed out that the hierarchical cluster analysis appeared to uncover features of categorical structure not revealed with the multidimensional scaling techniques and favored the prototype approach which utilized the hierarchical analysis (1987). They believed that subordinate-level emotions within each basic-level emotion serve to specify the intensities and details of eliciting context within the basic emotions or to indicate the mix of basic emotions.

Frijda (1986) proposed a new cognitive theory that seems to merge the psychoevolutionary theory and hierarchical theory. Frijda suggested that emotions appear to correspond with appraisal patterns, as well as to modes of action readiness. Emotions are caused by events appraised as favorable or harmful (pleasant - unpleasant) to an individual's concerns (goals, motives or sensitivities), thereby elicits states of action readiness. Modes of appraisal 
differentiate major dimensions of emotion and more subtle differences between emotions are characterized by action readiness aspects (Frijda et al., 1989).

Storm and Storm (1987) further explored the hierarchical model for semantic structure of emotion states and developed the emotion taxonomy. Using sorting, ranking, multidimensional scaling, and hierarchical clustering analyses, 61 groups of emotions were categorized into 20 categories of emotion, which were then classified into four major dimensions: positive, negative, arousal, and cognition salient. This taxonomy provided a relative differentiation or similarity of emotional states and the language used to describe them.

\section{Emotion Assessment Scales in Clinical Psychiatry Practice}

The early emotion scales were developed for usage in clinical psychiatry. Profile of Mood States (POMS) is an assessment of transient mood states, measuring six mood factors: tension - anxiety, depression - dejection, anger - hostility, vigor - activity, fatigue - inertia, and confusion - bewilderment. Sixty-five adjectives were identified and present on the scale to evaluate the affective mood state of an individual (McNair, Lorr, \& Droppleman, 1971). The items are evaluated by how an individual has been feeling, including at the present time. This scale is highly dominated by the negative descriptors for mood states with only one positive mood factor (vigor - activity). Multiple Affect Adjective Check List - Revised (MAACL-R scale) is an alternative to the POMS scale, also consists of five mood categories with a total of 135 mood descriptors: anxiety, depression, hostility, positive effect, and sensation seeking (Zuckerman \& Lubini, 1965,1985). Moods are assessed in two states: 'in general' for affective traits, and 'today' for day-to-day fluctuations. The assessment is done in the form of a checklist of terms and the terms are not scaled. This method is shown to differentiate patients with affective disorders on their personalities and emotions (http://www.edits.net/MAACL-R.html). Brunel University Mood Scale (BRUMS) is a shortened, modified version of the POMS scale; it consists of 24 items to assess six dimensions of mood previously developed in POMS (Terry, Lane, Lane, \& Keohane, 1999; Terry \& Lane, 2003). The BRUM scale is modified from POMS because of the need for an application with an adolescence population (young athletes; age < 18), and for a shorter version of the scale. Emotion terms are rated on a 5-point scale anchored by "not at all" (0) to "extremely" (4). This was developed mainly for use in the field of exercise psychology, focusing on exercise-induced mood change. Its validity has been demonstrated by 
Lane, Crone-Grant, \& Lane , 2002, among the 975 athletes who completed the mood questionnaire to assess mood states before and after exercise. In 2003, Terry, Lane, \& Fogarty further confirmed the validity of the scale by extending the usage from adolescent to adult populations.

Most scales discussed previously were developed in the field of psychology for clinical practice or used to assess, describe, and categorize general mood states in humans.

\section{Social Science}

Social science research further expanded the psychological theories and studies of emotions by pointing out limitations in the field of emotions. They hypothesized that emotion studies must be done within each culture and language since these elements are influential in how emotions are recognized, defined, and expressed. Scherer (2005) distinguished between the emotion types, proposing that the emotions occurring with consumption are classified as aesthetic emotions. These emotions are diffuse responses toward the intrinsic qualities of the objects or experiences, rather than the adaptive and action-oriented emotional responses for the increased survival tendencies. This definition agrees with Russell (1991) who summarized that upbringing, culture, and language shaped a person's emotional experiences towards stimuli (i.e., how emotions are classified and articulated). Russel indicated that words used to label each emotion may vary, to some extent, among cultures; and the individuals of different cultures could be affected, thus respond to the same sensory stimuli differently. The impacts of cultural differences on experienced emotions were further explored by Hartel and Hartel (2005) who suggested that the ways and intensities in which emotions are reported are based on the differing cultural upbringing. Averill (2004) noted that emotion labels from dissimilar languages could have different connotations, from which care must be taken when drawing inferences. Lindquist (2009) emphasized the important role of language in the field of emotion and stated that emotions stem from the basic psychological state with both hedonic and arousal properties. This state of feeling is then processed through the emotion concepts which are encoded by language. In-line with other researchers, Lindquist recognized that emotion responses arise from cultures and individual variability. Within a culture, however, language largely influences the experience of emotion by entailing the concept of that particular affective state. Taking the cultural and 
language issues into account, Cochrane (2009) proposed an eight-dimensional emotion model that effectively distinguishes emotion labels within and across cultures. He first identified the general concepts used to define the affective space and constructed a model based on the development and function of emotions. With that reasoning, this model claimed to also clarify distinction among ambiguous languages. The eight dimensions were comprised of: attractedrepulsed, powerful-weak, free-constrained, certain-uncertain, generalized-focused, future directed-past directed, enduring-sudden, and socially connected-disconnect.

Other researchers from various disciplines recognized and were in agreement on the limitations these factors present in the emotion research. Chrea, Valentin, Sulmont-Rosse, Ly Mai, Hoang Nguyen, \& Abdi (2004) addressed the four fundamental effects of culture underlying odor perception and affective state. Cultural experience dictates perceptual judgments, detection, recognition and identification, and acceptability. Others proposed that affective responses toward sensory stimuli were largely influenced by past experiences or exposures (Herz 2005; Chrea, Valentin, Sulmont-Rosse, Hoang Nguyen, \& Abdi, 2005).

\section{Health and Nutrition}

Researchers have studied the development of emotions related to food and what constitutes food choice and preference. Nutrition, consumption, and emotions are closely interrelated making it impossible to have one without the others. Food itself is the source of nutrition, toxin, pleasure, and an expression of social values (Rozin \& Vollmecke, 1986; Rozin, 1999). A review by Rozin and Vollmecke (1986) examined the subject of food likes and dislikes and discussed about the factors affecting the development of food preferences (biological, cultural, psychological, and interactions among the three factors). Influential elements that drive food selection were identified as sensory properties, anticipated consequences (e.g., healthy, beneficial, poisonous), and symbolic motivation. The mechanism of affective responses acquired through exposure and learning were also provided.

\section{Assessment Scale for Health and Food Choice}

Food choice has increasingly become a topic of interest because of how food affects people through various ways, including health and nutrition. In an attempt to explain the root of 
food intake and obesity, Birch (1999) introduced a theory of the origins and development of food preferences. This idea is similar to that of Rozin and Vollmecke (1986). Many scales have been developed to identify and understand the consumer attitude with regard to dietary behavior. For example, The Transtheoretical Model (TTM; originally developed to be used in a smoking intervention study) was adapted to assess eating behavior. TTM consists of five sequential stages a person experiences during a conversion of behavior (i.e., changing diet). This model identified the stage in which an individual belongs to and determined important variables that trigger the transition from one stage to the next (Horwath, 1999; Prochaska \& Norcross, 2001). The Heath and Taste Attitude Scale (HTAS) also was developed to examine the affective reaction related to nutrition (health) and sensory aspects of food (Roininen et al., 2001). Food Neophobia Scale (FNS) and Food Involvement Scale are two more examples of scales used in the health and nutrition research that apply an assessment of consumer attitude in order to understand consumer perception of food (Ritchey, Frank, Hursti, \& Tuorila, 2003; Bell \& Marshall, 2003)

The Food Choice Questionnaire (FCQ) may have been the first scale to comprehensively determine the multidimensional motives underlying food selection. FCQ identified nine factors, including mood and sensory appeal that are the determinants of food choice (Steptoe, Pollard, \& Wardle, 1995). Usage of FCQ was later applied and validated in several follow-up cross-cultural studies. Important food choice factors were identified for each segment of tested populations (Prescott, Young, O’Neill, Yau, \& Stevens, 2002; Eartmans, Victoir, Vansant, \& Van den Bergh, 2005; Eartman, Victoir, Notelaers, Vansant, \& Van den Bergh, 2006; Scheibehenne, Miesler, \& Todd, 2007). A group of researchers also proposed a modified design that claimed to be an enhanced general food motivation model (Fotopoulos, Krystallis, Vassallo, \& Pagiaslis, 2009).

Thus far in the area of nutrition, all constructed models and scales for food choice discussed only the idea of consumer's perception and attitude, and have not incorporated the assessment of emotional experiences elicited by food consumption. Gibson (2006, 2010), a nutritionist, recognized that sensory, psychological (mood), and physiological mechanisms drive emotional determinants of food selection. Instead of focusing on a consumer's attitude, he viewed sensory, emotion, and food preference to be interrelated, and emotional responses to food are dictated by sensory experiences and expectation levels. To better tailor foods for meeting 
consumer's emotional needs, he suggested future research needs to characterize the drivers underlying the connection between food choice and emotions.

\section{Consumer Research}

In the past decade, consumer researchers have taken interest in developing the connection between emotions and product consumption within the areas of marketing, product development, and sensory research.

\section{Marketing}

Consumer research has applied emotions to the marketing field and how advertisement affects consumers. The PAD (pleasure-arousal-dominance) scale was developed for measuring emotional responses to marketing stimuli, and more specifically the environmental settings. Application of this scale is limited because it was not designed to capture all elicited emotions, but only to measure those elicited by environmental stimuli (Richins, 1997). Edell and Burke (1987) investigated the affective component of consumer attitudes and explored the effect of feelings on consumer judgment and perception when viewing different advertising media. They found that a single ad can elevate both positive and negative feelings which influence the formation of attitude toward a brand/product; 52 emotions elicited by advertisements were proposed and classified into three groups: upbeat, warm, and negative feelings. These scholars emphasized that emotion is an individual's experience, and descriptors of emotion elicited across individuals (and a way to assess them) should be identified. Holbrook and Batra (1987) determined a range of emotional responses to various product consumptions and supported the role of emotions in mediating consumer reactions. These authors offered a list of 34 emotion terms that measure 12 types of hedonic responses to advertising (Batra \& Holbrook, 1990). Other researchers have also contributed to the area of emotions in relation with the product consumption experience. Strong interrelationships between emotions elicited by product experience and consumer satisfaction were demonstrated (Westbrook 1987; Westbrook \& Oliver, 1991). Several frameworks that link emotions, evaluation, and liking/satisfaction have been proposed and previously developed dimensionality theories of emotions (e.g., PANAS, Circumplex model, Utilitarian and hedonic dimensions) were also evaluated (Mano \& Oliver, 1993; Oliver, 1993; Havlena \& Holbrook, 1986). 
Although these studies have established the important role of emotions on product experience, and thus provided a foundation of emotion frameworks and scales, they do not focus on emotions experienced during product consumption.

\section{Consumption Experience}

Since the 1990's, the emotions related to product consumption and usage have received much attention and development through research focused on product development and sensory evaluation. The following models and scales have made an impact on the research field.

\section{The Consumption Emotions Set (CES)}

Richins (1997) reviewed emotion theories and measures previously used in the area of consumer research, and recognized the limitations those early studies presented. A comprehensive list of consumption-related emotions was then developed and validated for its usefulness. This study was completed in six studies. Study 1 employed an open-ended survey to capture the emotions consumers experienced during six different types of consumption situations. A preliminary set of 175 descriptors was generated. Study 2 asked respondents to rate the likelihood of their use of each descriptor to describe their feeling they experienced. Terms that were rated as 'not likely' by more than $5 \%$ of consumers were eliminated, leaving 129 terms in the set. Study 3 assessed how often each 129 emotions were experienced by consumers. Emotions reported to be experienced by less than $10 \%$ of respondents were removed, reducing the list to 97 terms. Study 4 evaluated the term redundancy by examining cooccurrence; consumers were asked to rate the extent a specified consumption situation made them feel each of the 97 descriptors, and the Multidimensional Scale (MDS) was used to determine descriptors with similar coordinates on the dimensions of the MDS solution. With further refinement, the Consumption Emotion Set (CES) was proposed - a set of 43 emotions classified into 16 dimensions with four additional descriptors were classified as 'others' (Figure 1.1). Studies 5 and 6 verified the appropriateness of this set to assess consumption-related emotions and differentiate feelings elicited by different consumption situations. CES provides future researchers with one of the most comprehensive sets of emotions evoked by various consumption experiences. 
Richins (1997) stated that CES is not intended to be an absolute assessment tool for emotions elicited by all consumptions. Rather, CES should serve as a baseline for further determining the appropriate assessment of consumption-related emotions.

\begin{tabular}{|c|c|c|c|}
\hline Anger & $\begin{array}{l}\text { Frustrated } \\
\text { Angry } \\
\text { Irritated }\end{array}$ & Peacefulness & $\begin{array}{l}\text { Calm } \\
\text { Peaceful }\end{array}$ \\
\hline Discontent & $\begin{array}{l}\text { Unfulfilled } \\
\text { Discontented }\end{array}$ & Contentment & $\begin{array}{l}\text { Contented } \\
\text { Fulfilled }\end{array}$ \\
\hline Worry & $\begin{array}{l}\text { Nervous } \\
\text { Worried } \\
\text { Tense }\end{array}$ & Optimism & $\begin{array}{l}\text { Optimistic } \\
\text { Encouraged } \\
\text { Hopeful }\end{array}$ \\
\hline Sadness & $\begin{array}{l}\text { Depressed } \\
\text { Sad } \\
\text { Miserable } \\
\end{array}$ & Joy & $\begin{array}{l}\text { Happy } \\
\text { Pleased } \\
\text { Joyful } \\
\end{array}$ \\
\hline Fear & $\begin{array}{l}\text { Scared } \\
\text { Afraid } \\
\text { Panicky } \\
\end{array}$ & Excitement & $\begin{array}{l}\text { Excited } \\
\text { Thrilled } \\
\text { Enthusiastic } \\
\end{array}$ \\
\hline Shame & $\begin{array}{l}\text { Embarrassed } \\
\text { Ashamed } \\
\text { Humiliated }\end{array}$ & Romantic Love & $\begin{array}{l}\text { Sexy } \\
\text { Romantic } \\
\text { Passionate } \\
\end{array}$ \\
\hline Loneliness & $\begin{array}{l}\text { Envious } \\
\text { Jealous } \\
\text { Lonely }\end{array}$ & Love & $\begin{array}{l}\text { Loving } \\
\text { Sentimental } \\
\text { Warm hearted }\end{array}$ \\
\hline & Homesick & Other items & Guilty \\
\hline Surprise & $\begin{array}{l}\text { Surprised } \\
\text { Amazed } \\
\text { Astonished }\end{array}$ & & $\begin{array}{l}\text { Proud } \\
\text { Eager } \\
\text { Relieved }\end{array}$ \\
\hline
\end{tabular}

Figure 1.1. The Consumption Emotion Set (CES; Richins, 1997)

\section{Hierarchical model of consumer emotions}

Laros and Steenkamp (2005) developed a hierarchical consumer emotion model by integrating various emotion theories and frameworks from the field of psychology and market/consumer research. A total of 173 negative emotions and 143 positive emotions were drawn from literature. They proposed a three-level hierarchical model (Figure 1.2): the superordinate level includes positive and negative affects, the basic level contains four negative 
(anger, fear, sadness, and shame) and four positive (contentment, happiness, love, and pride) emotions, and the subordinate level consists of specific emotions (CES - Richins, 1997).

\begin{tabular}{|cccccccc|}
\hline & \multicolumn{2}{l}{ Negative Affect } & & \multicolumn{3}{c|}{ Positive Affect } \\
Anger & Fear & Sadness & Shame & Contentment & Happiness & Love & Pride \\
Angry & Scared & Depressed & Embarrassed & Contented & Optimistic & Sexy & Pride \\
Frustrated & Afraid & Sad & Ashamed & Fulfilled & Encouraged & Romantic & \\
Irritated & Panicky & Miserable & Humiliated & Peaceful & Hopeful & Passionate & \\
Hostility & Nervous & Helpless & & Happy & Loving & \\
Unfulfilled & Worried & Nostalgia & & Pleased & Sentimental & \\
Discontented & Tense & Guilty & & Joyful & Warm-hearted \\
Envious & & & & Relieved & \\
Jealous & & & & Thrilled & \\
& & & & & & \\
& & & & & & & \\
\end{tabular}

Figure 1.2. Hierarchical model of consumer emotions (Laros \& Steenkamp, 2005)

This structural model was tested across food types (genetically-modified food, functional food, organic food, and regular food). Consumers were asked to rate whether they experienced these feelings from 'not at all' to 'very strongly'. However, the authors excluded two basic emotions (love and pride) and two specific emotions (envious and jealous) due to their inappropriateness in describing emotions evoked by food consumption. They indicated that the basic emotions provided in-depth information for better characterizing emotions consumers experienced.

\section{$\operatorname{PrEmo}{ }^{\circledR}$}

Desmet (2004) recognized that emotional response to the product experience is subtle and usually complex. Moreover, different culture and language influence how emotions are identified, defined, and expressed. To overcome these factors, he investigated both a non-verbal emotion assessment instrument, as well as, verbal instruments, and identified their limitations. Emotional responses elicited are difficult to measure because their nature is subtle (low intensity) and often mixed (more than one emotional response at the same time). A Product Emotion Measurement $(\operatorname{PrEmo} \AA)$-non-verbal self-report instrument - was developed using expressive cartoon animations to assess 14 emotions evoked by product design and experience. Seven of the 14 emotions measure positive feelings (desire, pleasant surprise, inspiration, amusement, 
admiration, satisfaction, and fascination) and the rest measure negative emotions (indignation, contempt, disgust, unpleasant, surprise, dissatisfaction, disappointment, and boredom). These 14 distinct emotions were selected from 347 emotion descriptors represented in Russell's Circumplex model (Russell, 1980)

PrEmo® has been validated for application across various populations. The major advantages of this tool are: 1) it only measures core, distinctive emotions elicited by the product experience, and 2) it can be used across populations of varying cultures and languages. Desmet (2004) also indicated that this instrument was designed to assess the emotions experienced toward static product design (2004), and the 14 expressions might not represent emotional reactions toward dynamic human product interaction (e.g. consumption experience). Regardless, this development presented a new opportunity for researchers to further explore the area of emotion study. Other facial scales that measure human expressions are Noldus Face Reader (2007; www.noldus.com) and Emotionomics (2007; www.sensorylogic.com), both of which contained a short list of emotions, similar to PrEmo®.

Desmet and Hekkert (2007) established a general framework of product experience that covers all emotions involved in every human-product interactions. The product experience framework consists of three dimensions: aesthetic experience (sensory characteristics), level of meaning (cognition and appraisal), and emotional experiences. Desmet, along with Schifferstein (2008), also investigated the emotions responses to food experiences and identified five sources of food emotions: sensory attributes, experienced consequences, anticipated consequences, individual meaning (personal/cultural), and actions of associated agents (2008). They observed in the study that positive emotions were reported more frequently than negative emotions, and thus reasoned it was because food products are designed to be satisfying and consumers select the food products they want to consume, hence a higher chance for a positive experience. They introduced this concept as "hedonic asymmetry" (Desmet \& Schifferstein, 2008).

\section{EsSense Profile ${ }^{\mathrm{TM}}$}

King and Meiselman (2009) built on the concept of hedonic asymmetry and proposed an emotion scale that can be applied towards assessment of feelings related to food consumption. A list of 39 emotion terms was created, and validated, to be able to differentiate consumer's

emotional experiences between and within food categories. The existing emotion terms (81 descriptors) from a previously developed standardized mood questionnaire (POMS and 
MAACL-R) were used as a starting point in the evaluation process. Identification, categorization, and selection of the 81 terms were done by consumers via the internet, central location tests, and a focus group. Consumers were asked to describe their most favorite and least favorite meal and/or food product. They were presented with a list of emotions and select terms to describe their feelings when consuming each product. The authors identified the higher usage frequency of the positive terms used to describe food consumption. Another set of consumers was asked to categorize emotions into positive, negative, both positive and negative, or neither positive nor negative. This step was to differentiate between terms having unambiguous meanings to consumers when compared with terms that were unclear, or could be interpreted in many ways. Then terms were selected based on 3 criteria: 1) frequency of use, a checklist questionnaire was employed to obtain the usage frequency of terms (at least 20\%); 2) terms must have clear categorization as positive or negative; and 3) suitability of the terms to describe emotions generated by food consumption. This was captured through consumer feedback. The final list contains 39 emotion terms.

The constructed emotion questionnaire was named the EsSense Profile ${ }^{\mathrm{TM}}$ (will be referred to as ESP) and was designed to measure emotion intensities using a 5-point intensity scale (Figure 1.3). The goal was to differentiate emotion experience among and within product categories. A 9-point hedonic scale was also added to assess overall acceptability of a product. The questionnaire was validated among five food categories (pizza, vanilla ice cream, fried chicken, and mashed potatoes with gravy) and was confirmed for its ability to discriminate among emotions experienced by different product categories. It was also validated for its differentiating power for product variations within the same food type (salty snack).

The emotions on the questionnaire were listed in alphabetical order. King and Meiselman (2009) had compared this alphabetical list of emotions to the randomized presentation, and indicated that order does not impact the results. The presentation of emotions was then kept in alphabetical order because the authors expected that it should enable respondents to quickly go through the ballot.

King and Meiselman (2009) identified usages of the ESP in the field of sensory analysis, product development and marketing. This scale serves as a tool to connect the three disciplines by providing a common emotion language for the development and marketing of the products that meet the consumer's emotional needs. 
How much you LIKE or DISLIKE (name of the product)?

\begin{tabular}{|c|c|c|c|c|c|c|c|c|}
\hline $\begin{array}{c}\text { Dislike } \\
\text { extremely }\end{array}$ & $\begin{array}{c}\text { Dislike } \\
\text { very much }\end{array}$ & $\begin{array}{c}\text { Dislike } \\
\text { moderately }\end{array}$ & $\begin{array}{c}\text { Dislike } \\
\text { slightly }\end{array}$ & $\begin{array}{c}\text { Neither } \\
\text { like } \\
\text { nor dislike }\end{array}$ & $\begin{array}{c}\text { Like } \\
\text { slightly }\end{array}$ & $\begin{array}{c}\text { Like } \\
\text { moderately }\end{array}$ & $\begin{array}{c}\text { Like } \\
\text { very much }\end{array}$ & $\begin{array}{c}\text { Like } \\
\text { extremely }\end{array}$ \\
\hline$\square$ & $\square$ & $\square$ & $\square$ & $\square$ & $\square$ & $\square$ & $\square$ & $\square$ \\
\hline
\end{tabular}

Please taste (product name) \#xxx now.

Below you will find words which describe different kinds of moods and feelings.

Using the terms listed, please describe how you FEEL RIGHT NOW. Please rate each feeling.

\begin{tabular}{|c|c|c|c|c|c|}
\hline Feelings & Not at all & Slightly & Moderately & Very & Extremely \\
\hline Active & 1 & 2 & 3 & 4 & 5 \\
\hline Adventurous & 1 & 2 & 3 & 4 & 5 \\
\hline Affectionate & 1 & 2 & 3 & 4 & 5 \\
\hline Aggressive & 1 & 2 & 3 & 4 & 5 \\
\hline Bored & 1 & 2 & 3 & 4 & 5 \\
\hline Calm & 1 & 2 & 3 & 4 & 5 \\
\hline Daring & 1 & 2 & 3 & 4 & 5 \\
\hline Disgusted & 1 & 2 & 3 & 4 & 5 \\
\hline Eager & 1 & 2 & 3 & 4 & 5 \\
\hline Energetic & 1 & 2 & 3 & 4 & 5 \\
\hline Enthusiastic & 1 & 2 & 3 & 4 & 5 \\
\hline Free & 1 & 2 & 3 & 4 & 5 \\
\hline Friendly & 1 & 2 & 3 & 4 & 5 \\
\hline Glad & 1 & 2 & 3 & 4 & 5 \\
\hline Good & 1 & 2 & 3 & 4 & 5 \\
\hline Good-natured & 1 & 2 & 3 & 4 & 5 \\
\hline Guilty & 1 & 2 & 3 & 4 & 5 \\
\hline Happy & 1 & 2 & 3 & 4 & 5 \\
\hline Interested & 1 & 2 & 3 & 4 & 5 \\
\hline Joyful & 1 & 2 & 3 & 4 & 5 \\
\hline Loving & 1 & 2 & 3 & 4 & 5 \\
\hline Merry & 1 & 2 & 3 & 4 & 5 \\
\hline Mild & 1 & 2 & 3 & 4 & 5 \\
\hline Nostalgic & 1 & 2 & 3 & 4 & 5 \\
\hline Peaceful & 1 & 2 & 3 & 4 & 5 \\
\hline Pleased & 1 & 2 & 3 & 4 & 5 \\
\hline Pleasant & 1 & 2 & 3 & 4 & 5 \\
\hline Polite & 1 & 2 & 3 & 4 & 5 \\
\hline Quiet & 1 & 2 & 3 & 4 & 5 \\
\hline Satisfied & 1 & 2 & 3 & 4 & 5 \\
\hline Secure & 1 & 2 & 3 & 4 & 5 \\
\hline Steady & 1 & 2 & 3 & 4 & 5 \\
\hline Tame & 1 & 2 & 3 & 4 & 5 \\
\hline Tender & 1 & 2 & 3 & 4 & 5 \\
\hline Understanding & 1 & 2 & 3 & 4 & 5 \\
\hline Warm & 1 & 2 & 3 & 4 & 5 \\
\hline Whole & 1 & 2 & 3 & 4 & 5 \\
\hline Wild & 1 & 2 & 3 & 4 & 5 \\
\hline Worried & 1 & 2 & 3 & 4 & 5 \\
\hline
\end{tabular}

Figure 1.3. EsSense Profile ${ }^{\mathrm{TM}}$ ballot for acceptability and emotion intensities (King \&

Meiselman, 2009) 


\section{Geneva Emotion and Odor Scale (GEOS)}

Emotion research has been growing and becoming more focused on specific product experiences, namely product design, food consumption, or in this case, odor stimulations. Chrea et al. (2009) described six latent factors used to measure affective feelings elicited by everyday odors via a two-staged study. First, participants described affective feelings elicited with exposure to a set of odorants. Factor analyses were employed to assess an appropriate set of emotion terms. Next, a larger group of participants validated this emotion model with a larger set of odorants. Confirmatory factor analysis was utilized to obtain the result. They proposed that a structure of emotional responses to odors differs from the frameworks suggested in earlier emotion theories. Emotions evoked by odor are explained by six factors that reflect the role of odors in humans. Thirty-six emotion terms were classified under the six factors: happiness/wellbeing, awe/sensuality, disgust/irritation, soothing/peacefulness, energizing/cooling, and sensory pleasure (Figure 1.4).

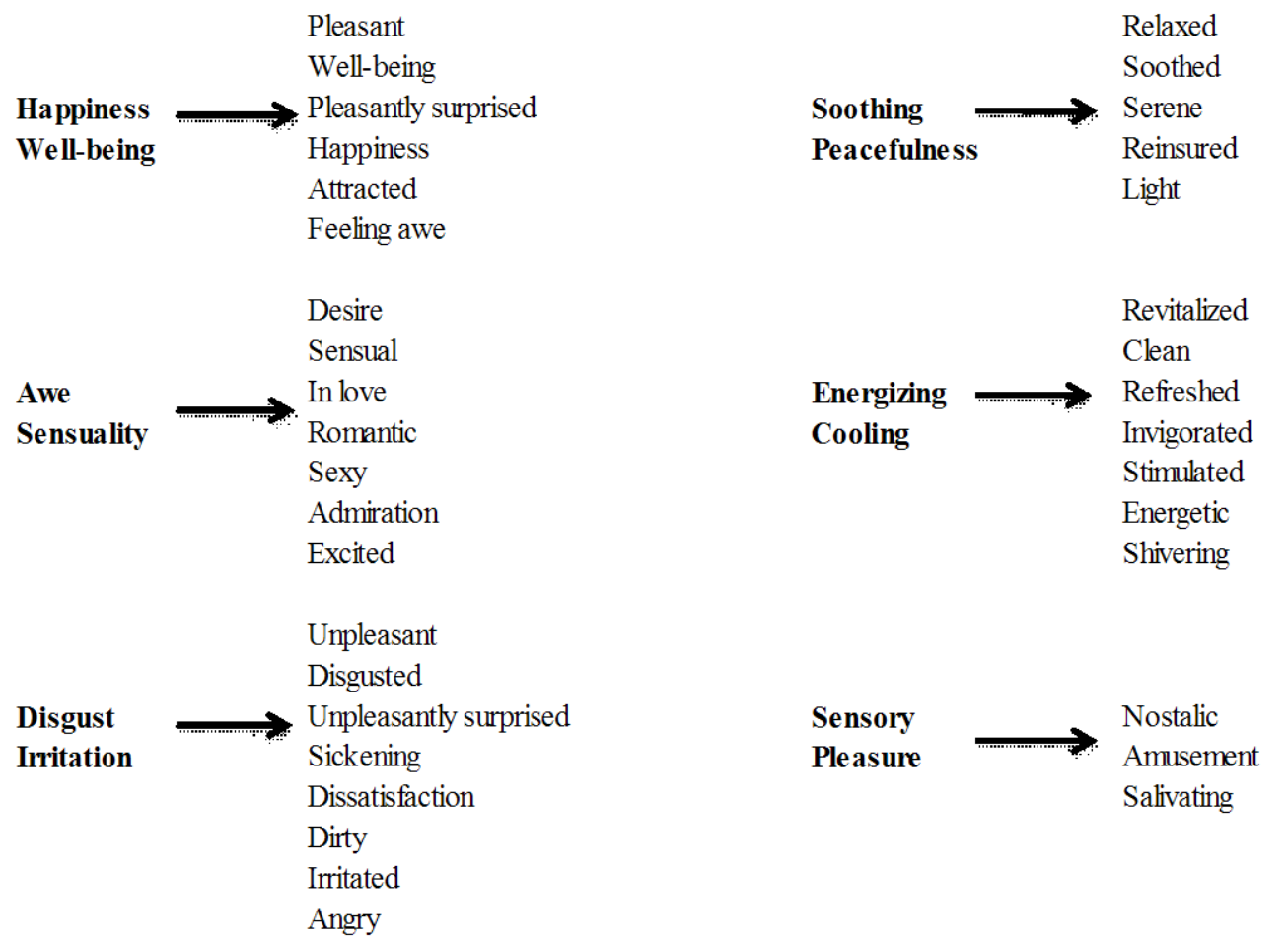

Figure 1.4. Six dimensions that describe affective feelings elicited by odors (GEOS; Chrea et al., 2009) 
Porcherot et al. (2010) further adapted GEOS to commercial fragranced and flavored products. They validated GEOS on its usefulness to differentiate feelings generated by stimulations of odors from products in the market. They also reduced the number of terms down to six dimensions consisting of three descriptors in order to ease the evaluation process for the consumers (happiness - well-being - pleasantly surprised; romantic - desire - in love; disgusted - irritated - unpleasantly surprised; relaxed - serene - reassured; nostalgic - amusement mouthwatering; energetic - invigorated - clean). It was found that the modified GEOS could provide addition information to assist product development as compared to the traditional consumer acceptability questions and was comparable to the original GEOS.

\section{Emotion and Coffee}

With the competitiveness in the market today, industries must seek in-depth understanding of the factors influencing consumers at an emotional level to sustain consumer satisfactions. Identifying the emotional elements that consumers experience and expect in a product provides a complete perspective on consumer affective behavior (behaviors influenced by emotions). These latent emotions are the foundation of likings and satisfaction.

Coffee is an important part of the American's routine. Therefore, understanding the latent emotions underlying coffee consumption is critical for the coffee industry. The US is the largest coffee market in the world. The Specialty Coffee Retailer (Bolton, 2009) reported that three out of four cups of coffee were home-brewed while over $80 \%$ of the money coffee drinkers spent was on foodservice coffee. One article (Anon, 2002) claimed that coffee drinkers select the roast of coffee based on the nature of the coffee establishment. Consumers tend to select lighter roast coffee when at coffee shops or breakfast diners, while they would choose darker roasts or espresso beverage when frequenting an upscale café or restaurant. Further exploration is needed in the arrays of emotions, attitudes or perceptions, and levels of involvement towards various coffee beverages that occur during the entire drinking experience from a wide range of coffee drinkers.

Each culture, drinking tradition, or language will influence the emotions associated with the drinking experience. Although some researchers argued that emotional expression and recognitions are biological and cognitive responses which are innate and universal (Plutchik, 1980; Thayer, 1978), the way the sensory stimuli affects one's emotions, or the way that 
responses are reported, varies among individuals of diverse cultural background and languages (Russell, 1991; Hartel \& Hartel, 2005; Averill, 2004). Other researchers agreed and suggested that past experience and exposure also influence affective responses (Herz, 2005; Chrea et al., 2005).

Coffee drinking cultures, which encompass the way coffee is brewed and drinking ritual, vary across different regions of the world. In some countries (especially in the Americas, the Middle East, and Europe) coffee is a drink for social gathering, which is why coffee houses are notably popular. In northern Europe, coffee parties (where coffee is served with homemade cake and pastries) are a common form of entertainment. In the early 1900's, the espresso beverage was born in Italy and has then changed the way Italians, and perhaps the rest of the world, drink coffee. Espresso is brewed using hot water and high pressure, in a shorter time period (Illy \& Viani, 2005). Espresso-based beverages resulted from this invention and have spread around the globe. Turkish coffee is brewed differently than most coffee; it is made with finely grounded roasted coffee beans and is brewed un-filtered in a long handled brass pot (ibrik). Sugar and cardamom sometimes are added to the coffee. In Asia, coffee is preferred light and sweet (Sullivan, 2010). Coffee is generally consumed at home in India, and small portions are prepared with milk, sugar, and some spices (Vikram, 2010). Instant coffee is commonly consumed at workplaces or at home in the Southeast Asian countries, which reflects the region's low disposable income (Kincheloe, 1993). Street side cafés are widely available where brewed coffee is served hot or iced, sometimes with sweetened condensed milk or sugar. Coffee houses became popular only in the past decade, along with the ready-to-drink coffee in cans. In Vietnam, brewed coffee is traditionally served in a cup partly filled with sweetened condensed milk and roasted chicory (Smith, 2010). In the U.S., hot drip coffee is the norm and is served in the form of a "bottomless" cup at breakfast diners. In the recent years, however, Americans have taken interests in the gourmet and specialty coffees (Bolton, 2009).

To understand the difference in coffee preferences among consumers, exploration is needed in the areas of emotions, attitudes or perceptions, and levels of involvement towards different coffee beverages from a wide range of coffee drinkers. A coffee emotion lexicon should be developed to measure the presence and intensities of the specific emotions that occur during the entire drinking experience of various coffee beverages.

This study investigated whether or not the emotion lexicon developed for coffee drinking 
provided additional information to the prior developed emotion questionnaire for the general food consumption (EsSense Profile ${ }^{\mathrm{TM}}$, King \& Meiselman, 2009). This study hypothesized that the emotion scale should be more product-specific instead of applying one universal scale for every food product. The EsSense Profile ${ }^{\mathrm{TM}}$ (ESP) was utilized as a foundation for the coffee drinking experience lexicon development because it has been validated on various food products, and it was able to discriminate among different categories and within the same food types. Currently, there is no research examining the possibility of capturing more signature emotions that may be imperative to the specific emotion experience elicited by coffee drinking. Therefore, our lexicon was generated by coffee consumers, following similar protocols outlined in the development of the ESP.

This study also explored the possibility of identifying key sensory descriptors that drive the emotion experiences during coffee consumption. The result provided a complete connection from the coffee sensory components to the elicited emotions by the sensory experience. Coffee manufacturers will be able to apply this information, which includes not only the overall acceptability of the sensory characters as before, but also how the sensory experiences stimulate positive or negative reactions from consumers. This should allow both marketing and product developers to better serve consumers based on the consumer's expectation of how they would like to feel during the entire drinking experience. 


\section{References}

Anonymous. (2002, August). Coffee - The Breakfast Journal. Nation's Restaurant News, 36, B1.

Averill, J.R. (2004). Everyday emotion: let me count the ways. Social Science Information, 43, 571-80.

Batra, R., \& Holbrook, M.B. (1990). Developing a typology of affective responses to advertising. Psychology \& Marketing, 7, 11-25.

Bell, R., \& Marshall, D.W. (2003). The construct of food involvement in behavioral research: Scale development and validation. Appetite, 40, 235-244.

Birch, L.L. (1999). Development of food preferences. Annual Review of Nutrition, 19, 41-62.

Bolton, D. (2009, August). Ten 2010 trends: Overview. Specialty Coffee Retailer, http://specialty-coffee.com. html (accessed October 2, 2010).

Chrea, C., Grandjean, D., Delplanque, S., Cayeux, I., Le Calve, B., Aymard, L., Velazco, M.I., Sander, D., \& Scherer, K.R. (2009). Mapping the semantic space for the subjective experience of emotional responses to odors. Chemical Senses, 34, 49-62.

Chrea, C., Valentin, D., Sulmont-Rosse, C., Hoang Nguyen, D., \& Abdi, H. (2005). Semantic, typicality and odor representation: a cross-cultural study. Chemical Senses, 30, 37-49.

Chrea, C., Valentin, D., Sulmont-Rosse, C., Ly Mai, H., Hoang Nguyen, D., \& Abdi, H. (2004). Culture and odor categorization: Agreement between culture depends upon the odors. Food Quality and Preference, 15, 669-679.

Cochrane, T. (2009). Eight dimensions for the emotions. Social Science Information, 48, 379420.

Crawford, J.R., \& Henry, J.D. (2004) The Positive and Negative Affect Schedule (PANAS): Construct validity, measurement properties and normative data in a large non-clinical sample. British Journal of Clinical Psychology, 43, 245-265.

Desmet, P.M.A. (2004). Measuring emotions: Development and application of an instrument to measure emotional responses to products. M.A. Blythe, K. Overbleeke, A.F. Monk \& P.C. Wright (Eds.), Funology: From Usability to Enjoyment. Dordrecht, The Netherland: Kluwer Academic Publishers.

Desmet, P.M.A., \& Hekkert, P. (2007). Framework of product experience. International Journal of Design, 1, 57-66.

Desmet, P.M.A., \& Schifferstein, H.N.J. (2008). Sources of positive and negative emotions in food experience. Appetite, 50, 290-301. 
Eartmans, A., Victoir, A., Vansant, G., \& Van den Bergh, O. (2005). Food-related personality traits, food choice motives and food intake: Mediator and moderator relationships. Food Quality and Preference, 16, 714-726.

Eartmans, A., Victoir, A., Notelaers, G., Vansant, G., \& Van den Bergh, O. (2006). The Food Choice Questionnaire: Factorial invariant over western urban populations? Food Quality and Preference, 17, 344-352.

Edell, J.A., \& Burke, M.C. (1987). The power of feelings in understanding advertising effects. Journal of Consumer Research, 14, 421-433.

Fotopoulus, C., Krystallis, A., Vassallo, M., \& Pagiaslis, A. (2009). Food Choice Questionnaire (FCQ) revisited. Suggestions for the development of an enhanced general food motivation model. Appetite, 52, 199-208.

Frijda, N.H. (1986). The emotions. Cambridge, England: Cambridge University Press.

Frijda, N.H. (1989). Relations among emotion, appraisal, and emotional action readiness. Journal of Personality and Social Psychology, 57, 212-228.

Gibson, E.L. (2006). Emotional influences on food choice: Sensory, physiological and psychological pathways. Physiology \& Behavior, 89, 53-61.

Gibson, E.L. (2010). Mood, emotions and food choice. In R. Shepherd \& M. Raats (Eds.), The Psychology of Food Choice (pp. 113-140). Oxfordshire, UK: CAB International.

Hartel, C.E.J., \& Hartel G.F. (2005). Cross-cultural differences in emotions: The why and how. Social Science Information, 44, 683-693.

Havlena W.J., \& Holbrook, M.B. (1986). The varieties of consumption experience: Comparing two typologies of emotion in consumer behavior. Journal of Consumer Research, 13, 394-404.

Herz, R.S. (2005.) Odor-associative learning and emotion: Effects on perception and behavior. Chemical Senses, 30, i250-i251.

Holbrook, M.B., \& Batra, R. (1987). Assessing the role of emotions as mediators of consumer responses to advertising. Journal of Consumer Research, 14, 404-420.

Horwath, C.C. (1999). Applying the transtheoretical model to eating behavior change: Challenges and opportunities. Nutrition Research Reviews, 12, 281-317.

Illy, A., \& Viani, R. (2005). Espresso coffee: The science of quality ( $2^{\text {nd }} \mathrm{ed}$.). London: Elsevier Academic Press.

Izard, C.E. (1977). Human emotions. New York: Plenum Press.

Izard, C.E. (2009). Emotion theory and research: highlights, unanswered questions, and emerging issues. The Annual Review of Psychology, 60, 1-25 
Kincheloe, D. (1993, July). Let the market drive you. Business Asia, 25, 12.

King, S.C., \& Meiselman, H.L. (2009) Development of a method to measure consumer emotions associated with foods. Food Quality and Preferences, 21, 168-177.

Lane, A.M., Crone-Grant, D., \& Lane, H.J. (2002). Mood changes following exercise. Perceptual and Motor Skills, 94, 732-734

Laros, F.J.M., \& Steenkamp, J.E.M. (2005). Emotion in consumer behavior: a hierarchical approach. Journal of Business Research, 58, 1437-1445.

Lindquist, K.A. (2009). Language is powerful. Emotion Review, 1, 16-18.

Mano, H., \& Oliver, R.L. (1993). Assessing the dimensionality and structure of the consumption experience: Evaluation, feeling, and satisfaction. Journal of Consumer Research, 20, 451-466.

Mayer, J.D., \& Gaschke, Y.N. (1988). The experience and meta-experience of mood. Journal of Personality and Social Psychology, 55, 102-111.

McNair, D. M., Lorr, M., \& Droppleman, L. F. (1971). Profile of mood states. San Diego: Educational and Industrial Testing Service.

Oliver, R.L. (1993). Cognitive, affective, and attribute bases of the satisfaction response. Journal of Consumer Research, 20, 418-430.

Plutchik, R. (1980). Emotions: A Psychoevolutionary Synthesis. New York: Harper \& Row.

Plutchik, R. (2001). The Nature of Emotions. American Scientist, 89, 344-350

Prescott, J., Young, O., O’Neill, L., Yau, N.J.N., \& Stevens, R. (2002). Motives for food choice: a comparison of consumers from Japan, Taiwan, Malaysia and New Zealand. Food Quality and Preference, 13, 489-495.

Prochaska, J.O., \& Norcross, J.C. (2001). Stage of change. Psychotherapy: Theory, Research, Practice, Training, 38, 443-448.

Porcherot, C., Delplanque, S., Raviot-Derrien, S., Le Calve, B., Chrea, C, Gaudreau, N., \& Cayeux, I. (2010). How do you feel when you smell this? Optimization of a verbal measurement of odor-elicited emotions. Food Quality and Preference, doi:10.1016/j.foodqual.2010.03.012.

Richins, M.L. (1997). Measuring emotions in the consumption experience. Journal of Consumer Research, 24, 127-146. 
Ritchey, P.N., Frank, R.A., Hursti, U., \& Tuorila, H. (2003). Validation and cross-national comparison of the food neophobia scale (FNS) using confirmatory factor analysis. Appetite, 40, 163-173.

Roininen, K., Tuorila, H., Zandstra, E.H., De Graaf, C., Vehkalahti, K., Stubenitsky, K., and Mela, D.J. (2001). Differences in health and taste attitudes and reported behavior among Finnish, Dutch, and British consumers: a cross-national validation of the Health and Taste Attitude Scales (HTAS), Appetite, 37, 33-45.

Rosch, E. (1973). On the internal structure of perceptual and semantic categories. In T.E. Moore (Ed.), Cognitive Development and the Acquisition of Language (pp. 111-144). New York: Academic Press.

Rosch, E. (1978). Principle of categorization. In E. Rosch \& B.B. Lloyd (Eds.), Cognition \& Categorization (pp. 27-48). Hillsdale, NJ: Erlbaum.

Rozin, P. (1999). Food is fundamental, fun, frightening, and far-reaching. Social Research, 66, 930.

Rozin, P., \& Vollmecke T.A. (1986). Food likes and dislikes. Annual Review of Nutrition, 6, 433-456.

Russell, J.A., \& Pratt, G. (1980) A description of the affective quality attributed to the environments. Journal of Personality and Social Psychology, 38, 311-322.

Russell, J.A. (1980). A circumplex model of affect. Journal of Personality and Social Psychology, 39, 1161-1178.

Russell, J.A. (1989) Affect grid: A single-item scale of pleasure and arousal. Journal of Personality and Social Psychology, 57, 493-502.

Russell, J.A. (1991). Culture and the categorization of emotions. Psychology Bulletin, 110, 426450 .

Scheibehenne, B., Miesler, L., \& Todd, P.M. (2007). Fast and frugal food choice: Uncovering individual decision heuristics. Appetite, 49, 578-589.

Scherer, K.R. (2005). What are emotions? And how can they be measured. Social Science Information, 44, 695-729.

Shaver, P., Shcwartz, J., Kirson, D., \& O’Connor, C. (1987). Emotion knowledge: Further exploration of a prototype approach. Journal of Personality and Social Psychology, 52, 1061-1086.

Steptoe, A., Pollard, T.M., \& Wardle, J. (1995). Development of a measure of the motives underlying the selection of food: the Food Choice Questionnaire. Appetite, 25, 267-284. 
Storm, C. \& Storm, T. (1987). A taxonomic study of the vocabulary of emotions. Journal of Personality and Social Psychology, 53, 805-816.

Sullivan, C.R. (2010, May). Coffee culture around the world. Retrieved from http://ezinearticles.com/?Coffee-Culture-Around-the-Wold\&id=4282795.

Terry, P.C. \& Lane, A.M. (2003) User Guide for the Brunel Mood Scale (BRUMS)

Terry, P.C., Lane, A.M., \& Fogarty, G.J. (2003). Construct validity of the Profile of Mood States - Adolescents for use with adults. Psychology of Sport and Exercise, 4, 125-139

Terry, P. C., Lane, A. M., Lane, H. J., \& Keohane, L. (1999). Development and validation of a mood measure for adolescents. Journal of Sports Sciences, 17, 861-872

Thayer, R.E. (1967). Measurement of activation through self-report. Psychological Reports, 20, $663-678$

Thayer, R.E. (1978). Toward a psychological theory of multidimensional activation (arousal). Motivation and Emotion, 2, 1-34

Thayer, R.E. (1989). The Biopsychology of Mood and Arousal. New York: Oxford University Press.

Vikram, S.S. (2010, August). Identifying the footprints. India Currents, 24, 60-61.

Watson, D., \& Tellegen, A. (1985). Toward a consensual structure of mood. Psychological Bulletin, 98, 219-235

Watson, D., Clark, L.A., \& Tellegen, A. (1988). Development and validation of brief measures of positive and negative affect: the PANAS scales. Journal of Personality and Social Psychology, 54, 1063-1070.

Westbrook, R.A. (1987). Product/consumption-based affective responses and post-purchase processes. Journal of Marketing Research, 24, 258-270.

Westbrook, R.A., \& Oliver, R.L. (1991). The dimensionality of consumption emotion patterns and consumer satisfaction. Journal of Consumer Research, 18, 84-91.

Zuckerman, M., \& Lubin, B. (1965). Manual for the multiple affect adjective check list. San Diego: Educational and Industrial Testing Service.

Zuckerman, M., \& Lubin, B. (1985). The multiple affect adjective check list revised. San Diego: Educational and Industrial Testing Service 


\title{
CHAPTER 2 - The Development of Emotion Lexicon for Coffee Drinking Experience
}

\begin{abstract}
To gain and retain competiveness, products must contain sensory characteristics that provide desirable emotional experiences. Consumer products are perceived via sensory aspects that stimulate emotional responses and cognition. Only a small number of emotion lexicons have been developed, and all having an objective of measuring affective responses for a wide range of product consumptions. It is not clear if those emotion scales for general consumption experience can uncover the deeper and distinct emotions created by specific products, especially those often consumed primarily for pleasure (e.g., coffee) rather than for nutritional values. The objective of this study was to develop an emotion lexicon that could be used to identify and measure feelings that occur with coffee drinking. We hypothesized that the lexicon could provide in-depth information on emotions evoked by the coffee drinking experience. Focus groups of coffee drinkers were held to generate emotion terms related to coffee drinking and then to eliminate redundant and ambiguous words. The emotion terminologies were then refined again by asking 48 coffee drinkers to choose emotions (check-all-that-apply) that were applicable to how they were feeling while they consumed two coffee samples. The final list of emotion terms is composed of 86 items, 47 generated by coffee drinkers and 39 terms from a general emotion lexicon.
\end{abstract}

\section{Introduction}

Emotion has always been an integral part of human beings. Many theorists have attempted to understand emotions by classifying emotions into structural model, framework, or dimensions, and identifying the stimuli or components underlying each emotional response (Thayer, 1978; Plutchik, 1980; Russell, 1980; Watson \& Tellegen, 1985; Mayer \& Gaschke, 1988; Rosch, 1978; Frijda, 1986; Izard, 1977). Scherer (2005) has defined emotion as "an episode of interrelated, synchronized change in the states of all or most of the five organismic subsystems in response to the evaluation of an external or internal stimulus event as relevant to 
major concerns of the organism." This suggests that the external experiences human perceive through the five senses assert influential impact on emotional reactions.

In the past, most companies have competed against each other by developing new products with matching or superior values, nutritional and sensorial aspects (Kim \& Mauborgne, 1999). Nowadays, there is an increase in awareness of the linkage between emotions and human cognitive behavior. With today's myriad of choices in the market for the consumers, industries must seek in-depth understanding of the factors influencing consumers at the emotional level to sustain consumer satisfactions. Identifying the emotional elements that the consumers experience and expect in a product through sensory elements provides a complete perspective on consumer affective behavior (i.e., behaviors influenced by emotions). Those emotions probably are the underlying dimensions for liking and satisfaction (Thomson, Crocker, \& Marketo, 2010). From the 1990's, emotions associated with product consumption have been investigated. Researchers have attempted to capture terminologies associated with positive emotions and develop comprehensive lists of emotions associated with consumption experiences. For example, Laros \& Steenkamp (2005) provided a model for consumer emotions proposing 33 emotion terms using terms drawn from previous literature. Rousset, Deiss, Juilard, Schlich, \& Droit-Volet (2005) developed an extensive emotion list where 70 terms were validated to describe emotions experienced by French women while consuming meat and other food products. Desmet \& Schifferstein (2008) proposed a way to measure complex emotions in product design using a non-verbal, cross-cultural tool called PrEmo® (Product Emotion Measurement Tool). PrEmo® consists of 14 animation characters expressing 7 positive and 7 negative emotions. It is used primarily to assess intensity of each elicited emotion by the product's appearance. King \& Meiselman (2009) created the EsSense Profile ${ }^{\mathrm{TM}}$ (ESP), using adjectives from POMS (Profile of Mood State; McNair, Lorr, \& Droppleman, 1971) and MAACL-R (Multiple Affect Adjective Check List - Revised; Zuckerman \& Lubin, 1965 \& $1985)$ to measure affective responses for product consumption. Terms were created and validated based on clarity and usage frequency; this ensured application for a wide range of products. The final lexicon consisted of 39 emotions to represent consumer emotional connection with the product consumption.

Most emotion lexicons may have certain limitations because they were created based on the entire consumption experience, generating emotion terms that were versatile and broad but 
less product specific. An example of a more specific emotion lexicon is that created by Chrea $e t$ al. (2009) for odors in everyday life. Because odors can be important elicitors of emotion experiences that differ from emotions experienced across a wide range of products, a lexicon for odors recently was developed to assess emotional responses.

Another issue is that many emotion lexicons were verified and modified based on how frequently respondents used each term to describe their emotions towards different types of food. This ensured the emotion terms created were applicable to a wide range of food. However, each food category may have its own unique sensory properties and functional purposes that could induce deeper and more distinctive sets of emotions worth exploring.

A sensory lexicon is a set of words used to describe sensory characteristics found in a specific product category, which aids in product development process (Seo, Lee, \& Hwang, 2009; Dooley, Adhikari, \& Chambers, 2009) and many sensory lexicons have been published for a variety of products (e.g. Civille, Lapsley, Huang, Yada, \& Seltsam, 2010; Drake, Yates, \& Drake, 2010; Talavera-Bianchi, Chambers, \& Chambers, 2010). Although a lexicon of sensory attributes is used to identify attributes that appeal to various consumer markets, it is critical to explore what emotions these sensory attributes elicited in order to have a better understanding of consumer segmentations and decision making processes. An emotion lexicon can measure emotions or feelings induced by product consumption through the sensory experiences. However, similar to a sensory lexicon, an emotion lexicon may need to be created for a specific product category, particularly those products consumed for pleasure and not for nutritional purposes (e.g., coffee, tea, wine, chocolate).

Coffee is one of the most popular beverages enjoyed by diverse global populations, and understanding the relationship between coffee preferences and emotional experiences among consumers might be beneficial for researchers and product marketers across the globe. Coffee drinking cultures, which encompass the way coffee is brewed and the drinking ritual, vary across different regions of the world. In the Americas, the Middle East, and Europe, coffee is commonly consumed in a café and is a drink for social interaction (Sullivan, 2010) whereas in Asia, instant coffee is commonly consumed at workplaces or at home (Kincheloe, 1993). Further exploration is needed to determine the array of emotions, perceptions, and levels of involvement towards various coffee beverages occurring during the entire drinking experience from a wide range of coffee drinkers at various locations. The purpose of this study was to 
develop a lexicon of emotion terms that could be used to examine the emotional responses to coffee. In this study, ESP was utilized as a baseline for the emotion term development because it is the most recent food-related emotion lexicon that has been validated for use in various product categories (King \& Meiselman, 2009; Bell, 2009)

\section{Materials and Methods}

The study was conducted in 3 parts - 1) four mini-focus groups of heavy coffee users and one mini-focus group of medium to light coffee users, thus generating emotion adjectives related to the coffee drinking experience; 2) two focus groups with heavy coffee users to fine-tune the generated list of emotion terms; 3) terminology refinement using both medium and heavy coffee drinkers to further refine the list of emotions by usage frequency. The emotion scale (ESP) developed by King and Meiselman (2009) was used as a basis while developing the coffee drinking emotion lexicon.

\section{Mini-Focus Group Interviews}

To identify emotion terms related to the coffee drinking experience, coffee consumers were interviewed. We segmented coffee experiences into four situations based on locations consumers drink coffee beverages: coffee shop/restaurant, home, office/work, or on-the-go (i.e., drive-thru, vending machine, convenience stores).

\section{Recruitment}

Participants were selected based on their weekly coffee consumption frequency. For the purpose of this study, those who drank coffee at least once daily and visited a coffee shop at least once a week were classified as 'heavy users'. Those who drank coffee 3-5 times a week were classified as 'medium users', and those who drink coffee 1-2 times a week were classified as 'light users'. Heavy users were grouped into four quads (four participants in each quad). One additional quad was comprised of light and medium users. Light/medium users were included to provide a complete perspective of the emotions experienced by all coffee drinkers during the consumption. Age and gender were balanced within each quad as much as possible. Each quad contained two males and two females within the age groups of 18-35 and 36-70. 


\section{Methodology}

Five, 60-min focus groups were conducted in a reserved study room at two local coffee shops. Each interview session was audio-recorded and respondents were aware of the recording for research purposes.

The moderator began the sessions by asking participants to identify their favorite coffee beverage, explaining what they enjoy about it, and what sensory characteristics or feelings they hope to get from the beverage. The moderator then asked general questions about their coffee drinking routine and habits, followed by more specific questions about their feelings during the coffee drinking experience and definitions of a 'good' cup and a 'bad' cup of coffee. Participants were asked to think about the emotions they feel when drinking a 'good' versus a 'bad' cup of coffee, then focus on the drinking experiences at specific locations (home, the coffee shop/restaurant, work, or on-the-go). Participants discussed drinking habits at specific locations, coffee selection criteria, and reasons why they chose to drink coffee from/at certain locations. The moderator asked each participant to describe drinking experiences at that location, including good, bad, most enjoyable, and worst experiences. Questions asking whether there were any drawbacks to coffee drinking in general or to having coffee at a specific place were added as appropriate. Interviews continued in this manner until all locations were covered or the moderator deemed no additional emotion terms could be generated from the groups. At each session's end, each participant was asked to sum up the whole coffee drinking experience into 3-5 words for each good and bad experience. If needed, what the person meant by a term was discussed and further clarified. The moderator's guide for the mini-focus group's interviews is illustrated in Appendix A.

\section{Focus Group Interviews}

To fine-tune the emotions list, heavy users were selected to participate in this phase of the study. Two focus groups were conducted to determine the appropriate selection of terms.

\section{Recruitment}

Heavy users were recruited. Consumers who had previously participated in the mini focus groups could not participate in this session. Each group consisted of 5-6 heavy users where age (18-65) and gender were equally distributed in each focus group. 


\section{Methodology}

Two 90-min focus group sessions were conducted at the Sensory Analysis Center,

Kansas State University, Manhattan, KS, USA. An audio-tape recorder and a note taker were used for recording the interview sessions. Coffee was provided to create a coffee drinking experience, but drinking was optional. The emotion terms generated by the mini-focus groups and words from the ESP (King \& Meiselman, 2009) were combined into one list and were presented to participants in both focus groups. Discussion began with the moderator asking participants to reflect on their favorite coffee beverage, and highlight or circle emotion terms from the list to represent how they felt. Next, the participants were asked to remember bad experiences with coffee (time they received a 'bad' cup of coffee) and highlight emotion terms to represent those feelings. Last, the moderator asked participants to think about their regular, everyday experiences with the coffee they drink on a daily basis; then highlight terms to represent their feelings. Participants were also instructed to write down any other emotion terms they believed described their coffee drinking experiences, but was absent from the list.

The moderator wrote all emotion terms on the whiteboard as each participant read aloud the terms they had chosen. Each adjective was then tallied. Discussion then focused on the coverage of emotion terms chosen, redundancy of terms, clarity of meanings, and appropriateness of any additional terms provided by participants. The moderator also asked the groups to generate single adjectives to describe larger phrases noted from previous mini-focus groups. To end the session, the groups were asked to discuss the coffee drinking experience at home, coffee shop, work, and on-the-go in order to generate any additional emotions that may have been overlooked. The format of the interview is illustrated in Appendix B.

\section{Terminology Refinement}

To refine the lexicon further a small group of coffee users $(\mathrm{N}=48)$ were recruited to select relevant terms that describe the emotions related to coffee drinking while drinking 2 coffee samples. Check-all-that-apply (CATA) method (Dooley, Lee, \& Meullenet, 2010; Ares, Barreiro, Deliza, Gimenez, \& Gambaro, 2010) was used to record the responses. The use of a smaller number of consumers with CATA method was utilized because our objective was to capture as many emotions related to coffee drinking. At this stage a large consumer evaluation was not necessary to achieve the objective of terminology refinement. Also, this lexicon is 
intended to be a starting point and will need to be adapted to other cultures and countries. Term selection was based on frequency of usage and clarity of the terms.

\section{Recruitment}

Medium to heavy users were recruited. Equal numbers of male and female users age 1870 participated. Prior to this stage, our focus was mainly on heavy coffee users but in this stage we also included medium users to achieve broader representation of coffee consumers.

\section{Samples and preparation}

Two types of ground coffee were used to represent light and dark roast varieties: Starbucks Coffee ${ }^{\circledR}$ Breakfast Blend (Starbucks Coffee Company; Seattle, WA, USA) and Dunkin’ Donuts ${ }^{\circledR}$ Dark Roast (The Procter \& Gamble Company; Cincinnati, OH, USA). Each coffee was brewed separately (model 169058 coffee maker; General Electric Company, Fairfield, CT, USA) following the user manual's instructions. Coffee machines were filled with reversed osmosis, de-ionized, carbon-filtered water and each brewed coffee was filtered through Melitta coffee filter \#4 (Melitta USA, Inc.; Clearwater, FL, USA). Coffee samples were brewed during each session and served within 5 min of brewing. Coffee was served in individual 165 mL ceramic cups with a saucer (Econo Rim, Syracuse China; Lyncourt, NY, USA), individual packets of half \& half (Land O'Lakes Half \& Half UHT Processed Creamer - single servings 11.25 mL; Land O’ Lakes, Inc.; St. Paul, MN, USA), sugar (Serene Sysco sugar packet - 2.83 g; Sysco Corp.; Houston, TX, USA), and sweetener (Sweet N’ Low® Zero Calorie Sweetener; Cumberland Packing Corp.; Brooklyn, NY, USA) were provided as needed. The approximate serving temperatures were approximately $70^{\circ} \mathrm{C}$.

\section{Methodology}

Three sessions of central-location-test were conducted in a food-safe laboratory room. Sixteen consumers participated in each session. A CATA questionnaire containing the 118 emotion terms fine-tuned by the focus group sessions was given to each consumer to evaluate each coffee. Participants were instructed to drink the sample provided and check the terms describing their feelings at that moment. They were encouraged to take their time and continue drinking the beverage as they went though the list of emotions. After participants finished their first sample, the second sample was served. Participants then repeated the same process. 
Serving order was balanced to reduce bias from order effects (Meilgaard, Civille, \& Carr, 2007; Resurreccion, 1998).

Once the data were compiled, the usage frequency for each emotion was summed up and terms with frequency of 10 or lower were eliminated, except for terms belonging to the ESP (King \& Meiselman, 2009). A frequency of 10 was chosen as a cut-off point in order to eliminate emotions that may be irrelevant but still include as many emotion terms that were experienced by the majority of coffee users as possible. Higher cut-off points such as 15 or 20 might have eliminated some relevant terms.

\section{Results and Discussions}

\section{Mini-Focus Group Interviews}

From the group discussions, 95 emotion terms related to coffee drinking experience were generated in addition to the 39 emotion terms from the ESP (King \& Meiselman, 2009), resulting in a total of 134 words (Table 2.1). Of the 39 terms in ESP, 26 terms were mentioned by the participants. The remaining 13 terms (affectionate, daring, free, good-natured, joyful, loving, merry, mild, quiet, tame, tender, wild, and worried) were not mentioned.

Table 2.1. Emotion terms generated by mini-focus group discussions

\begin{tabular}{|c|c|c|c|c|}
\hline ACTIVE & CONFUSED & GLAD & MILD & SECURE \\
\hline ADAPTIVE & CONNECTED & GOOD & MOTIVATED & SICK \\
\hline ADVENTUROUS & CONTENT & GOOD-NATURED & NOSTALGIC & SIMPLE \\
\hline AFFECTIONATE & $\mathrm{COZY}$ & GROUCHY & OBLIGATED & SOCIAL \\
\hline AGGRESSIVE & CULTURED & GUILTY & OFF-BALANCE & SOOTHING \\
\hline ALERT & DARING & GUILTY PLEASURE & PEACEFUL & SPECIAL \\
\hline ANGRY & DEPRESSED & HABIT & PLEASANT & SPIRITUAL \\
\hline ANNOYED & DESIRABLE & HAPPY & PLEASED & SPONTANEOUS \\
\hline ANTICIPATING & DISAPPOINTED & HISTORICAL & POLITE & STABLE \\
\hline ASSURED & DISGUSTED & HOME & POWERFUL & STEADY \\
\hline ATTENTIVE & EAGER & IMPULSIVE & PRESTIGIOUS & STRESSFUL \\
\hline AWAKE & EDUCATED & IN CONTROL & PRODUCTIVE & SURPRISED \\
\hline BALANCED & EMPOWERING & INDEPENDENT & PUT-TOGETHER & TAME \\
\hline BETRAYED & ENERGETIC & INTERESTED & QUIET & TENDER \\
\hline BOOSTED & ENTHUSIASTIC & INTRIGUED & READY & TIRED \\
\hline
\end{tabular}




\begin{tabular}{|l|l|l|l|l|}
\hline BORED & EXCITED & RELAXED & TOLERATED \\
\hline BUZZED & EXPERIMENTAL & JITTERY & RELIEVE & TRIGGERED \\
\hline CALM & FAMILIAR & JOLTED & REMINISCE & UNDERSTANDING \\
\hline CHILLED & FAMILY & JOYFUL & RESTED & UPSET \\
\hline CLEAR MINDED & FESTIVE & JUDGMENTAL & REWARDED & WARM \\
\hline COLLECTED & FIT & JUMP START & RISKY & WASTED \\
\hline COMFORTABLE & FOCUSED & LAZY & RITUAL & WHOLE \\
\hline COMFORTED & FREE & LITERATE & ROUTINE & WILD \\
\hline COMMITTED & FRIENDLY & LONGEVITY & SAD & WITTY \\
\hline COMPLETE & FRUSTRATED & LOST & SAFE & WORLDLY \\
\hline COMPLEX & FULFILLED & LOVING & SATISFIED & WORRIED \\
\hline COMRADELY & FUN & MERRY & SEASONAL & \\
\hline \hline
\end{tabular}

*Bold terms are from the ESP

\section{Focus Group Interviews}

The focus group sessions reduced the list of emotions from 134 terms down to 118 terms (Table 2.2). The participants, by consensus, removed several terms from the list as discussed below. Adaptive, historical, judgmental, literate, longevity, seasonal, and wasted were not considered to be emotions or feelings and, thus, were eliminated from the list. Comradely, prestigious, spiritual, witty, and worldly were removed because the groups felt these words were complicated. Complex, connected, fit, and involved were decided to be too vague for the list. Last, chilled was removed because of informality and redundancy with other emotion terms.

All of 39 terms from the ESP (King \& Meiselman, 2009) were retained in the list of terms. However, participants did not give the same definitions for free and warm. Free was deemed confusing because it was not clear whether it referred to monetary value or spiritual meaning. Some participants thought that warm referred to temperature, while others defined it as friendly and warm-hearted. Regardless, these terms were included in the list because they were words from the original emotion set in the ESP. These terms may need more in-depth study.

Table 2.2. List of emotion terms fine-tuned by the focus group discussions

\begin{tabular}{|l|l|l|l|l|}
\hline ACTIVE & COZY & FUN & MOTIVATED & SAFE \\
\hline ADVENTUROUS & CULTURED & GLAD & NERVOUS & SATISFIED \\
\hline AFFECTIONATE & CURIOUS & GOOD & NOSTALGIC & SECURE \\
\hline AGGRESSIVE & DARING & GOOD-NATURED & OBLIGATED & SICK \\
\hline ALERT & DEPRESSED & GROUCHY & OFF-BALANCE & SIMPLE \\
\hline ANGRY & DESIRABLE & GUILTY & PEACEFUL & SOCIAL \\
\hline
\end{tabular}




\begin{tabular}{|l|l|l|l|l|}
\hline ANNOYED & DIFFERENT & GUILTY PLEASURE & PLEASANT & SOOTHING \\
\hline ANTICIPATED & DISAPPOINTED & HABIT & PLEASED & SPECIAL \\
\hline ASSURED & DISGUSTED & HAPPY & POLITE & SPONTANEOUS \\
\hline ATTENTIVE & EAGER & HOME & POWERFUL & STABLE \\
\hline AWAKE & EDUCATED & IMPULSIVE & PUT-TOGCTIVE & STEADY \\
\hline BALANCED & EMPOWERING & IN CONTROL & STRESSFUL \\
\hline BETRAYED & ENERGETIC & INDEPENDENT & QUIET & SURPRISED \\
\hline BOOSTED & ENTHUSIASTIC & INTERESTED & READY & TAME \\
\hline BORED & EXCITED & INTRIGUED & RELAXED & TENDER \\
\hline BUZZZED & EXPERIMENTAL & JITTERY & RELIEVED & TIRED \\
\hline CALM & FAMILIAR & JOLTED & REMINISCE & TOLERATED \\
\hline CLEAR MINDED & FAMILY & JOYFUL & RESTED & UNDERSTANDING \\
\hline COLLECTED & FESTIVE & JUMP START & REWARDED & UPSET \\
\hline COMFORTABLE & FOCUSED & LAZY & RISKY & WARM \\
\hline COMFORTED & FREE & LOST & RITUAL & WHOLE \\
\hline COMPLETE & FRIENDLY & LOVING & ROUTINE & WILD \\
\hline CONFUSED & FRUSTRATED & MERRY & SAD & WORRIED \\
\hline CONTENT & FULFILLED & MILD & & \\
\hline \hline
\end{tabular}

*Bold terms are from the ESP

\section{Terminology Refinement}

The 118 emotion terms from the focus group sessions were further refined to 86 emotion terms (Table 2.3), including the 39 terms from ESP, by coffee users through the CATA method. CATA offered the identification of various emotions experienced by coffee users of different preferences, and most terms with the frequency lower than 10 were removed. However, certain negative emotions with frequency lower than 10 were retained. Most negative emotions received fairly low usage frequencies, which was logical because the two coffee products tested were well-known brands that were already established in the market, suggesting that they have reasonable consumer acceptance rates. However, consumption experience might not always be positive. Therefore, upset, frustrated, sad, disappointed, annoyed, and grouchy were selected to remain on the list since they represented common negative feelings induced during consumption experience. Jolted and nervous also were included, although the frequencies were low because jolted represented an intense feeling that could be induced by a particularly strong cup of coffee, and nervous represented the opposite anchor term for calm or relaxed. Both of these terms also could represent the physiological or psychological reaction to caffeine - or maybe participants were jolted by the hot coffee or nervous being in the test. 
Table 2.3. Final list of emotion term refined by the check-all-that-apply method

\begin{tabular}{|c|c|c|c|c|}
\hline ACTIVE & CULTURED & GLAD & MOTIVATED & SATISFIED \\
\hline ADVENTUROUS & CURIOUS & GOOD & NERVOUS & SECURE \\
\hline AFFECTIONATE & DARING & GOOD-NATURED & NOSTALGIC & SOCIAL \\
\hline AGGRESSIVE & DISAPPOINTED & GROUCHY & OFF-BALANCE & SOOTHING \\
\hline ALERT & DISGUSTED & GUILTY & PEACEFUL & SPECIAL \\
\hline ANNOYED & EAGER & HAPPY & PLEASANT & STABLE \\
\hline ATTENTIVE & EDUCATED & HOME & PLEASED & STEADY \\
\hline AWAKE & EMPOWERING & IN CONTROL & POLITE & TAME \\
\hline BALANCED & ENERGETIC & INDEPENDENT & PRODUCTIVE & TENDER \\
\hline BOOSTED & ENTHUSIASTIC & INTERESTED & QUIET & TIRED \\
\hline BORED & EXCITED & INTRIGUED & READY & TOLERATED \\
\hline CALM & EXPERIMENTAL & JOLTED & RELAXED & UNDERSTANDING \\
\hline CLEAR MINDED & FOCUSED & JOYFUL & RELIEVED & UPSET \\
\hline COLLECTED & FREE & JUMP START & RESTED & WARM \\
\hline COMFORTABLE & FRIENDLY & LOVING & REWARDED & WHOLE \\
\hline COMFORTED & FRUSTRATED & MERRY & SAD & WILD \\
\hline CONTENT & FULLFILLED & MILD & SAFE & WORRIED \\
\hline $\mathrm{COZY}$ & FUN & & & \\
\hline
\end{tabular}

*Bold terms are from the ESP

The emotion terms with frequencies above 10 were kept on the list with some exceptions, and similar terms were merged or removed. Assured, complete, and put-together were also taken out because they had similar meanings with secure, whole, and in control, respectively. Impulsive and spontaneous were eliminated because they shared similar meaning with adventurous (Merriam-Webster, 2010). Habit, family, familiar, and routine are behavioral terms rather than psychological, thus, did not belong on the list. Different and simple were too broad and vague; obligated may not be related to the whole coffee drinking experience but instead to the nature of product-testing procedures, which could be misleading; stressful can be related to a number of other influencing factors, and therefore, too complicated to measure.

The refined list of 86 emotion terms (Table 2.3) is a starting point for further research on emotional aspects of coffee drinking in diverse cultures. Language and culture influence how emotions are experienced, categorized, defined, and expressed. Therefore, the developed emotion lexicon for coffee drinking experience may need to be modified to fit each country's or each culture's drinking tradition and appropriate emotion language (Russell, 1991). Some 
researchers argue that emotional expressions and recognitions are biological and cognitive responses that are innate and universal (Plutchik, 1980; Thayer, 1978). However, this study showed that simply identifying a product of focus can influence the selection of terms to express the emotional experiences related to a specific product. Scherer (2005) suggested that the emotions associated with product consumption are classified as aesthetic emotions, which should vary from one culture to another. This is in-line with Russell (1991) who summarized that cultural upbringing shapes a person's emotional experiences towards various sensory stimuli. To some extent words used to label emotions will vary among different cultures, therefore, we are surmising that our lexicon would change depending on the culture and language of the target population. Similar assertions have been made by other researchers (Hartel \& Hartel, 2005; Averill, 2004; Herz, 2005; Chrea, Valentin, Sulmont-Rosse, Hoang Nguyen, \& Abdi, 2005). Another factor that could influence this emotion lexicon is the difference in the coffee drinking cultures in various regions of the world. For example, espresso was created in Italy in the early 1900's and has changed the way Italians, and perhaps the rest of Europe, view and drink coffee. It is brewed using hot water and high pressure, in a shorter time period (Illy \& Viani, 2005). Turkish coffee is brewed differently than most coffee - finely ground roasted coffee bean is brewed unfiltered in a long handle brass pot (ibrik). Sugar and cardamom are sometimes added to the coffee. In Asia, coffee is preferred light and sweet (Sullivan, 2010). Sweetened condensed milk is commonly added to the brewed coffee. In India coffee is generally prepared in small portions with milk, sugar, and some spices (Vikram, 2010). In the U.S., hot drip coffee is the norm and is served in the form of "bottomless" cup at breakfast diners (Bolton, 2009), although the gourmet and specialty coffee have become more popular among the American in the recent years. All these different drinking rituals and traditions will impact the emotional response and consequently, there might be changes in the developed lexicon when it is adapted by other researchers.

\section{Conclusion}

The ESP lexicon served as a good baseline for the development of emotion lexicon for the coffee drinking experience. Forty-seven additional emotions related to coffee drinking were generated by this study and could provide researchers with more in-depth information about 
feelings that distinguish one coffee from another. The coffee drinking experiences might differ depending on the coffee products and drinking cultures. The lexicon should be modified as appropriate as each culture, drinking tradition, or language will influence the emotions associated with the drinking experience. The emotion lexicon from our study provides a starting point for further emotion research on coffee drinking experience. It is not intended to be a final lexicon that can be used universally without modifications. In Chapter 3, the lexicon was further verified using 6 varieties of coffee and 94 coffee users in Manhattan, KS, USA. This was to identify the core emotions that are important to the coffee drinking experience. 


\section{References}

Ares, G., Barreiro, C., Deliza, R., Gimenez, A., \& Gambaro, A. (2010). Application of a checkall-that-apply question to the development of chocolate milk desserts. Journal of Sensory Studies, 25, 67-86.

Averill, J.R. (2004). Everyday emotion: let me count the ways. Social Science Information, 43, 571-80.

Bell, B.E. (2009). The effects of spice blends in an apple-based extruded cereal-like product: Maximizing flavor and health. [Master of Science Thesis]. Manhattan, KS, USA: Kansas State University. 85 p. Available from: University Catalog, Manhattan, KS

Bolton, D. 2009. Ten 2010 trends: Overview. http://www.specialtycoffee.com/ME2/Audiences/dirmod.asp?sid=\&nm=\&type=MultiPublishing\&mod=Publis hingTitles\&mid=8F3A7027421841978F18BE895F87F791\&tier=4\&id=94146FA8B1914 E8FA6DEA7022467D978\&AudId=464620AE3F20454894C8CB7CEF72A481 (accessed October 2, 2010).

Chrea, C., Grandjean, D., Delplanque, S., Cayeux, I., Le Calve, B., Aymard, L., Velazco, M.I., Sander, D., \& Scherer, K.R. (2009). Mapping the semantic space for the subjective experience of emotional responses to odors. Chemical Senses, 34, 49-62.

Chrea, C., Valentin, D., Sulmont-Rosse, C., Hoang Nguyen, D., \& Abdi, H. (2005). Semantic, typicality and odor representation: a cross-cultural study. Chemical Senses, 30, 37-49.

Civille, G.V., Lapsley, K., Huang, G., Yada, S. \& Seltsam, J. (2010). Development of an almond lexicon to assess the sensory properties of almond varieties. Journal of Sensory Studies, 25, 146-162.

Desmet, P.M.A. \& Schifferstein, H.N.J. (2008). Sources of positive and negative emotions in food experience. Appetite, 50, 290-301.

Dooley, L., Adhikari, K., \& Chambers, E. IV. (2009). A general lexicon for sensory analysis of texture and appearance of lip products. Journal of Sensory Studies, 24, 581-600

Dooley, L., Lee, Y.S., \& Meullenet, J.F. (2010). The application of check-all-that-apply (CATA) consumer profiling to preference mapping of vanilla ice cream and its comparison to classical external preference mapping. Food Quality and Preference, 21, 394-401.

Drake, S.L., Yates, M.D., \& Drake, M.A. (2010). Development of a flavor lexicon for processed and imitation cheeses. Journal of Sensory Studies, 24, 720-739.

Frijda, N.H. (1986). The emotions. Cambridge, England: Cambridge University Press.

Hartel, C.E.J., \& Hartel G.F. (2005). Cross-cultural differences in emotions: The why and how. Social Science Information, 44, 683-693.

Herz, R.S. (2005.) Odor-associative learning and emotion: Effects on perception and behavior. Chemical Senses, 30, i250-i251. 
Illy, A., \& Viani, R. (2005). Espresso coffee: The science of quality ( $2^{\text {nd }}$ ed.). London: Elsevier Academic Press.

Izard, C.E. (1977). Human emotions. New York: Plenum Press.

Kim, W.C., \& Mauborgne, R. (1999). Creating new market space [Reprint 99105]. Harvard Business Review (January/February), 83-93.

Kincheloe, D. (1993). Let the market drive you. Business Asia, 25(15), 12.

King, S.C., \& Meiselman, H.L. (2009). Development of a method to measure consumer emotions associated with foods. Food Quality and Preference, 21, 168-177.

Laros, F. and Steenkemp, J. (2005). Emotion in consumer behavior: A hierarchical approach. Journal of Business Research, 58, 1437-1445.

Mayer, J.D., \& Gaschke, Y.N. (1988). The experience and meta-experience of mood. Journal of Personality and Social Psychology, 55, 102-111.

McNair, D.M., Lorr, M., \& Droppleman, L.F. (1971). Profile of mood states. San Diego: Educational and Industrial Testing Service.

Meilgaard, M.C., Civille, G.V., \& Carr, B.T. 2007. Factors Influencing Sensory Verdict Sensory Evaluation Techniques (4 ${ }^{\text {th }}$ Ed.). (pp. 39-43). Boca Raton, FL: CRC Press,

Merriam-Webster. (2010). Merriam-Webster Online Thesaurus, http://www.merriamwebster.com (accessed October 2, 2010).

Plutchik, R. (1980). Emotions: A Psychoevolutionary Synthesis. New York: Harper \& Row.

Resurreccion, A.V.A. (1998). Test Procedures. Consumer Sensory Testing for Product Development. (pp. 43-66). Gaithersburg, MD: An Aspen Publication.

Rosch, E. (1978). Principle of categorization. In E. Rosch \& B.B. Lloyd (Eds.), Cognition \& Categorization (pp. 27-48). Hillsdale, NJ: Erlbaum.

Rousset, S., Deiss, V., Juillard, E., Schlich, P., \& Droit-Volet, S. (2005). Emotions generated by meat and other food products in women. British Journal of Nutrition, 94, 609-619.

Russell, J.A. (1980). A circumplex model of affect. Journal Personality and Social Psychology, 39, 1161-1178.

Russell, J.A. (1991). Culture and the categorization of emotions. Psychology Bulletin, 110, 426450.

Scherer, K.R. (2005). What are emotions? And how can they be measured. Social Science Information, 44, 695-729.

Seo, H-S, Lee, S-Y., \& Hwang, I. (2009). Development of sensory attribute pool of brewed coffee. Journal of Sensory Studies, 24, 11-132 
Sullivan, C.R. 2010. Coffee culture around the world. http://ezinearticles.com/?Coffee-CultureAround-the-Wold\&id=4282795 (accessed October 15, 2010).

Talavera-Bianchi, M., Chambers, E. IV, \& Chambers, D.H. 2010. Lexicon to describe flavor of fresh leafy vegetables. Journal of Sensory Studies, 24, 163-183.

Thayer, R.E. (1978). Toward a psychological theory of multidimensional activation (arousal). Motivation and Emotion, 2, 1-34

Thomson, D.M.H., Crocker, C., \& Marketo, C.G. (2010). Linking sensory characteristics to emotions: an example using dark chocolate. Food Quality and Preference. doi:10.1016/j.foodqual.2010.04.011.

Vikram, S.S. (2010). Identifying the footprints. India Currents, 24(8), 60-61.

Watson, D., \& Tellegen, A. (1985). Toward a consensual structure of mood. Psychology Bulletin, 98, 219-235.

Zuckerman, M., \& Lubin, B. (1965). Manual for the multiple affect adjective check list. San Diego, CA: Educational and Industrial Testing Service.

Zuckerman, M., \& Lubin, B. (1985). The multiple affect adjective check list revised. San Diego, CA: Educational and Industrial Testing Service. 


\section{CHAPTER 3 - Examining the "coffee drinking experience" emotion lexicon}

This study evaluated and refined the previously developed emotion lexicon for measuring emotions elicited by the coffee drinking experience. In the previous study, 86 emotion terms were proposed, consisting of 47 terms identified by coffee drinkers and the 39 emotion terms from a recently developed emotion lexicon for foods. Six coffees were tested with 94 consumers in this study to determine the set of terms from the lexicon elicited in the consumers by the samples. The emotion questionnaire containing all the 86 terms was administered twice - before and during coffee drinking on a 5 -point scale $(1=$ not at all to $5=$ extremely). Overall acceptability of the samples was also asked in the study. The consumers were clustered into 6 clusters using the overall acceptability scores. Stepwise regression analysis with forward selection was done on the entire data set, by each consumer cluster, and by each coffee sample to identify the important emotion terms for prediction of coffee preference. Overall acceptability scores were used as response variable in the model, and emotion scores were the independent variables. Forty-four emotion terms were selected from this process and used to examine the emotion profile of each consumer cluster. Emotion profiles for each coffee sample within the consumer cluster were distinct. The product-emotion bi-plots demonstrated that each consumer cluster responded differently to the coffees they rated the highest. This indicates that each group of coffee drinkers sought different affective feelings from the drinking experience. This study also disclosed additional set of emotions describing an active and task-oriented mental state that could be useful for future application. It is evident that a complex product like coffee may need a specific emotion lexicon to uncover more information about how different coffee samples impact emotional responses from diverse coffee drinkers.

\section{Introduction}

With the competitiveness in the market today, it is no longer sufficient to evaluate product performance only by measuring overall liking on the sensory properties of a product. The level of emotional responses elicited by the sensorial experience during the product consumption is also vital. Today's consumers seek for emotional experiences they receive from a product via sensory perception (Thomson, 2010) and recently researchers have become more 
aware of the connection between the sensory perception and the emotional experiences elicited during the product consumption. King and Meiselman (2009) validated EsSense Profile ${ }^{\mathrm{TM}}$ (ESP) scale developed by themselves on various food products and were able to discriminate different categories and those within the same category. ESP consists of 39 emotion terms related to general product consumption is currently utilized by several food and beverage industries. Recently, a Geneva Emotion and Odor Scale (GEOS) was developed to measure affective feelings that respond to olfactory stimulation in a French speaking population (Chrea et al. 2009). GEOS contains 36 emotion terms classified into six dimensions: sensuality, relaxation, pleasant feeling, refreshment, sensory pleasure, and unpleasant feeling.

Currently, the present emotion scales available for public use were developed for general food and beverage consumption. However, consumers seek different sensations from food products and preferences for these sensations could change depending on time of day, situation, cultures and tradition, or context of consumption (Chrea et al., 2009; Labbe et al., 2009; Scherer, 2005; Herz, 2005; Hartel \& Hartel, 2005; Russell, 1991). Even different food varieties from the same category provide individuals with a wide range of sensory stimulations that arouse different emotions. Considering the uniqueness of distinct foods and beverages, it is plausible that an emotion scale especially developed for a specific product may be able to provide profound information on the deeper emotions underlining the consumption experiences.

Coffee is the one of the most popular beverages, enjoyed by all population of different cultures and the U.S. is the largest coffee market in the world. According to the International Coffee Organization (C, 1.3 billion Kg of coffee was consumed by Americans in 2009, which was over half of the entire coffee consumption of all European countries (2.4 billion $\mathrm{Kg}$ ). It is evident that coffee is an important part of an American's routine. To have a better understanding of coffee consumers' acceptances and consumption behaviors, it is critical to explore the emotions elicited by the different sensory characteristics of various coffee beverages. The emotions that occur during the coffee drinking experiences should be identified and measured, through the development of an emotion lexicon. Using ESP as a baseline, Bhumiratana \& others (submitted) explored this proposition and 86 emotion terms related to the coffee drinking experience was proposed. It was necessary that this list of 86 coffee-related emotions be evaluated and refined further through a range of coffee samples and sizable group of coffee drinkers. The main objectives of this study were 1) to provide a compact list of relevant terms 
that sufficiently describe the emotional experience during coffee drinking, and 2) to apply this list of relevant emotions to assess the emotion profiles of different segments of coffee users in and around Manhattan, Kansas, USA.

\section{Materials and Methods}

\section{Coffee Samples}

Six single-serve coffee samples were selected (K-Cup® Keurig, Inc.; Reading, MA, USA) to represent the range of roast levels from light to dark (Table 3.1). Single-serve coffee was used in this study to enable the randomized design of products among consumers and ensure similar serving temperature.

We covered the range of roast levels from light to dark and included coffee samples from various growing regions, as well as some organic certified samples. Six samples were selected based on those criteria so that at least one of the six samples would be the representative of individual likes or dislikes. All coffee samples were stored at room temperature $\left(20{ }^{\circ} \mathrm{C}\right)$ until testing and were used in the study within 6 weeks of delivery.

Table 3.1 List of coffee samples

\begin{tabular}{|c|c|c|c|}
\hline Brand & Type/Blend & Roast level & $\begin{array}{l}\text { Additional } \\
\text { information }\end{array}$ \\
\hline Green Mountain ${ }^{\circledR}$ & Breakfast & Light & \\
\hline Green Mountain ${ }^{\circledR}$ & Nantucket & Medium & \\
\hline Green Mountain ${ }^{\circledR}$ & Sumatra Reserved & Dark & $\begin{array}{l}\text { Fair Trade Certified }{ }^{\mathrm{TM}} \text {, } \\
\text { Organic }\end{array}$ \\
\hline Tully's & Kona & Medium & \\
\hline Tully's & Italian Roast & Dark & \\
\hline Newman's Own® Organic & Special Blend & Medium/Dark & $\begin{array}{l}\text { Fair Trade Certified }{ }^{\mathrm{TM}} \text {, } \\
\text { Organic }\end{array}$ \\
\hline
\end{tabular}




\section{Consumer Hedonic Testing}

Ninety-six consumers were recruited from various sources (Sensory Analysis Center's consumer database and local coffee shops) in Manhattan, KS, USA area based on their weekly coffee consumption (at least 3 times a week). All participants had no food allergies, were between ages 18-70, and were balanced within genders as much as possible. The participants composed of $66.6 \%$ females and $33.3 \%$ males.

To maximize the holistic experience of drinking coffee, each consumer evaluated one coffee sample per testing day at the Hoffman Lounge in Justin Hall, Kansas State University. The lounge setting, to some extent, simulated the coffee shop atmosphere where participants could choose where they would like to sit and drink their coffee on their own terms, with some passer-by or students presence around the area. Each consumer visited the facility to taste coffee 2 days per week for 3 weeks to complete to evaluate all 6 coffee samples in a balanced complete block design. Each session lasted approximately $30 \mathrm{~min}$ and order of sample presentation was balanced using Williams-modified Latin Square design to eliminate bias (Meilgaard, Civille, \& Carr, 2007; Resurreccion, 1998).

On the first day of testing, consumers were asked if they would like to add creamer (Hiland Ultra-Pasteurized Half \& Half - single servings 11.25mL; Hiland Dairy; Springfield, MO, USA), sugar (Serene Sysco sugar packet - 2.83 g; Sysco Corp., Houston, TX, USA), or sweetener (Sweet N' Low ${ }^{\circledR}$ Zero Calorie Sweetener; Cumberland Packing Corp.; Brooklyn, NY, USA) to their coffee. The information was recorded and the same amounts of creamer, sugar, and/or sweetener were provided to the consumers with their coffee all 6 times.

Participants were given a 2-page questionnaire (Appendix C) and their consumer numbers at the beginning of each visit. The format of the questionnaire was the same as that used in ESP, except for the list of the emotion terms. Participants were instructed to fill out the first page and rate their current feelings on a numerical 5-pt scale with anchor descriptors $(1=$ not at all; 2 = slightly; 3 = moderately; $4=$ very much; 5 = extremely) prior to drinking the coffee sample. The emotion state before coffee consumption was assessed because the emotional impact of food product depends on a persons' initial psychological state (Gibson, 2006; Macht, Roth, \& Ellgring, 2002). Once the coffee was served, the participant tasted the coffee and rated their overall liking of the sample, followed by evaluated the intensity of each emotion. The overall liking question was rated on a 9-point hedonic scale with anchors from dislike extremely 
to like extremely. Consumers were encouraged to rate their feelings as they drank the coffee without rushing through the process; however, they were not required to finish the cup of coffee. The questionnaires were collected at the end of each session.

At the last visit, upon completion of the evaluation, one-page demographic ballots (Appendix D) were given to participants to complete, which included the information on the age, gender, education background, annual income, and coffee drinking preference/habits.

\section{Sample Preparation and Serving}

Keurig® Special Edition B60 Brewing System (Keurig®, Inc.; Reading, MA, USA) was used to brew the single serve K-Cup® coffee samples. The machine was cleaned following the user's manual instructions. The water reservoir was filled with reverse osmosis, de-ionized, carbon filtered water. The designated K-Cup was placed in the machine and $157.5 \mathrm{~mL}$ of coffee was selected to brew into a ceramic mug (Econo Rim, Syracuse China; Lyncourt, NY, USA). The K-Cup was removed and discarded immediately after the brewing cycle was completed. Coffee was served immediately and the server reminded the consumer to use all of the creamer/sugar/sweetener requested.

\section{Statistical Analyses}

Out of 96 consumers, 94 were subjected to cluster analysis based on liking scores. Two consumers were taken out of the analysis because they failed to rate their liking on one sample. The CLUSTER procedure using Wards clustering method (minimum variance method) was utilized in SAS® (version 9.2; SAS Institute; Cary, NC, USA) for this purpose. Hierarchical dendogram and cubic clustering criterion were plotted to help decide the number of consumer clusters.

The emotion ratings prior to the coffee evaluation were subtracted from the emotion ratings during the evaluation before analyzing the data. The differences were labeled as emotion rating scores and further analyzed. Stepwise regression analysis using forward selection was conducted on the on the entire data set using PROC REG procedure in SAS®. A significance level of 0.20 was used to determine significant emotion terms (independent variables) that predicted liking scores (response variable) for each coffee sample. There is high variability in consumer data, meaning the probability of detecting differences among products is lower (Berger \& Hsieh, 2005; O’Mahony, 1986), therefore the higher level of significance $(\alpha=0.20)$ was 
chosen. Stepwise regression analysis using forward selection was also conducted on consumer clusters to identify emotion terms that can predict coffee preferences (coffee liking scores) for each cluster. The same procedure was also performed on data for each coffee sample in order to capture all relevant emotions. The terms significant at $\alpha=0.20$ in at least 3 consumer clusters or 3 coffee samples were selected. Figure 3.1 explains the flow chart of the stepwise selection process.

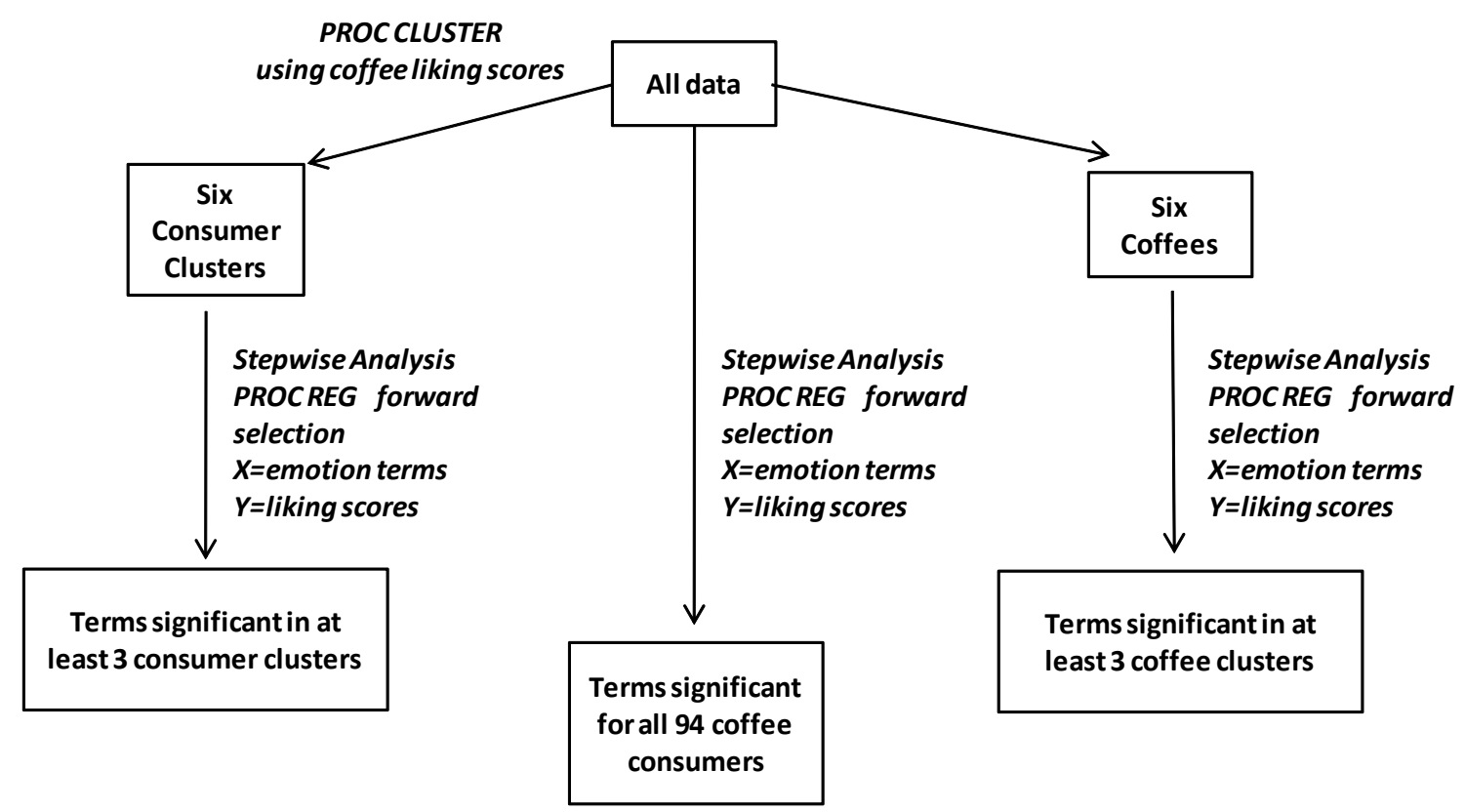

Figure 3.1. Flow Chart for Data Analysis

Once the appropriate set of emotions related to coffee drinking experience was established, principle component analysis was performed on each consumer cluster to verify the ability to discriminate among the coffee samples and to examine the insights revealed by the emotion profiles generated by the coffee drinking experience (Unscrambler® 9.8; Camo Software A/S, Oslo, Norway).

\section{Results and Discussions}

The cluster analysis yielded 6 clusters of consumers and the average liking scores of the coffee samples for each of the clusters is shown in Table 3.2. 
Table 3.2. Average liking scores for each consumer cluster and coffee sample

\begin{tabular}{|l|l|l|l|l|l|l|}
\hline Cluster & Breakfast & Italian & Kona & Nantucket & Newman & Sumatra \\
\hline C1 $(\mathrm{n}=20)$ & $7.7^{\mathrm{ns}}$ & $6.9^{\mathrm{ns}}$ & $7.3^{\mathrm{ns}}$ & $7.7^{\mathrm{ns}}$ & $7.5^{\mathrm{ns}}$ & $7.2^{\mathrm{ns}}$ \\
\hline $\mathrm{C} 2(\mathrm{n}=17)$ & $4.4^{\mathrm{c}}$ & $6.9^{\mathrm{ab}}$ & $6.7^{\mathrm{ab}}$ & $6.1^{\mathrm{b}}$ & $7.2^{\mathrm{a}}$ & $6.5^{\mathrm{ab}}$ \\
\hline C3 $(\mathrm{n}=24)$ & $7.0^{\mathrm{a}}$ & $6.0^{\mathrm{b}}$ & $5.8^{\mathrm{b}}$ & $7.5^{\mathrm{a}}$ & $5.3^{\mathrm{b}}$ & $3.7^{\mathrm{c}}$ \\
\hline C4 $(\mathrm{n}=13)$ & $4.6^{\mathrm{b}}$ & $3.5^{\mathrm{c}}$ & $5.7^{\mathrm{a}}$ & $6.0^{\mathrm{a}}$ & $5.5^{\mathrm{ab}}$ & $5.4^{\mathrm{ab}}$ \\
\hline C5 $(\mathrm{n}=10)$ & $7.1^{\mathrm{a}}$ & $3.3^{\mathrm{bc}}$ & $3.5^{\mathrm{b}}$ & $4.1^{\mathrm{b}}$ & $2.2^{\mathrm{cd}}$ & $2.1^{\mathrm{d}}$ \\
\hline C6 $(\mathrm{n}=10)$ & $5.9^{\mathrm{ab}}$ & $6.4^{\mathrm{ab}}$ & $7.0^{\mathrm{a}}$ & $3.6^{\mathrm{c}}$ & $5.1^{\mathrm{b}}$ & $6.1^{\mathrm{ab}}$ \\
\hline
\end{tabular}

Means within each cluster with different letters are significantly different $(P<0.05)$

Each cluster was described according to its average liking scores for each coffee sample. The 20 consumers belonging to Cluster 1 appeared to like all coffee samples and rated all samples equally. The 17 consumers in Cluster 2 showed significant dislike towards Breakfast. Cluster 3's 24 consumers liked Breakfast and Nantucket, while disliking Sumatra. Kona and Nantucket received the highest scores that were significantly higher than Breakfast and Italian in Cluster 4, even though the cluster's 13 consumers did not have positive preference for any of the coffees (all samples received average liking scores of 6 or lower). The 10 consumers in Cluster 5 liked Breakfast, but gave low ratings for the remaining coffees, showing strong dislikes towards Newman and Sumatra. The ten coffee drinkers in cluster 6 liked Kona and Italian, while Nantucket received the lowest liking score.

\section{Selecting the relevant emotions}

Stepwise regression analysis using forward selection at a significance level of 0.20 was completed on all 94 consumers, producing 25 emotions that were significant $(\mathrm{p}<0.20)$ for prediction of coffee liking (Model $\mathrm{R}^{2}=0.31$, Appendix E). The low model $\mathrm{R}^{2}$ was expected because consumers have a wide range of preferences. Therefore, it is difficult to determine variables that predict the pattern for liking in a diverse group of consumers. The same analyses were performed on the six consumer clusters, and on each coffee sample. This was to capture any additional emotions not identified as significant $(\mathrm{p}<0.20)$ in predicting coffee preference in a population with high variability $(\mathrm{n}=94)$. Looking at consumers by clusters allowed the selection of emotions that are used to characterize each coffee in a group where preferences in coffee were similar, thus, higher model $\mathrm{R}^{2}$ for prediction of liking is possible. Lists of emotions selected as significant $(p<0.20)$ for the model for each cluster and coffee sample were shown in 
Appendices G and H. Not all terms chosen by the Stepwise regression analyses were identified as important for the model. In the model which cumulative partial $\mathrm{R}^{2}$ reached 0.90 , additional emotion terms were discarded unless they contributed at least 0.01 of partial $\mathrm{R}^{2}$ to the model. Table Table 3.3 shows all selected terms from the Stepwise regression analyses of the entire data set, consumer clusters, and coffee clusters.

The relevant emotion set is a combination of the emotion terms that were significant $(\mathrm{p}<0.20)$ in predicting preferences in the entire set of consumers ( 25 terms), and the terms significant $(\mathrm{p}<0.20)$ in at least three consumer clusters $(10$ terms $)$ and/or three product clusters (25 terms). This was to ensure that every possible relevant emotion term was captured. The resulting list contains of 44 emotions identified as influential parameters in describing the coffee drinking experience (CDE). The 44 terms consisted of 17 emotions from ESP and 27 emotions generated by coffee drinkers (Table 3.3). 
Table 3.3. Lists of emotions derived from the coffee drinking experiences (CDE)

\begin{tabular}{|c|c|c|c|}
\hline $\begin{array}{l}\text { Significant emotions from the } \\
\text { entire data set }\end{array}$ & $\begin{array}{l}\text { Significant emotions in at least } \\
3 \text { consumer clusters }\end{array}$ & $\begin{array}{l}\text { Significant emotions in at least } \\
3 \text { coffee samples }\end{array}$ & 44 CDE Emotions \\
\hline & & Active* $^{*}$ & Active* \\
\hline \multirow[t]{2}{*}{ Annoyed } & & Annoyed & Annoyed \\
\hline & Awake & & Awake \\
\hline Balanced & & Balanced & Balanced \\
\hline \multirow[t]{3}{*}{ Boosted } & & & Boosted \\
\hline & Bored* & Bored* & Bored* \\
\hline & Clear minded & & Clear minded \\
\hline Comfortable & & Comfortable & Comfortable \\
\hline Content & & & Content \\
\hline Curious & & & Curious \\
\hline Disappointed & & & Disappointed \\
\hline \multirow[t]{2}{*}{ Disgusted* } & & Disgusted* & Disgusted* \\
\hline & & Educated & Educated \\
\hline \multirow[t]{6}{*}{ Empowering } & & & Empowering \\
\hline & Energetic* & & Energetic* \\
\hline & Free* & Free* & Free* \\
\hline & Fulfilling & Fulfilling & Fulfilling \\
\hline & & Fun & Fun \\
\hline & & Good & Good* \\
\hline Grouchy & & Grouchy & Grouchy \\
\hline Guilty* & & & Guilty* \\
\hline In control & & & In control \\
\hline \multirow[t]{2}{*}{ Jolted } & & & Jolted \\
\hline & & Joyful* & Joyful* \\
\hline Jump start & & & Jump start \\
\hline \multirow{3}{*}{ Merry* } & & Merry* & Merry* \\
\hline & & Motivated & Motivated \\
\hline & Nervous & & Nervous \\
\hline Off-balance & & Off-balance & Off-balance \\
\hline \multirow[t]{2}{*}{ Peaceful* } & & & Peaceful* \\
\hline & & Pleasant* & Pleasant* \\
\hline \multirow[t]{3}{*}{ Pleased* } & & & Pleased* \\
\hline & & Productive & Productive \\
\hline & & Relaxed & Relaxed \\
\hline \multirow[t]{2}{*}{ Rested } & & & Rested \\
\hline & & Rewarded & Rewarded \\
\hline Satisfied* & Satisfied* & Satisfied* & Satisfied* \\
\hline Social & & & Social \\
\hline \multirow[t]{2}{*}{ Soothing } & & Soothing & Soothing \\
\hline & Special & Special & Special \\
\hline Understanding* & & & Understanding* \\
\hline Warm* & & Warm* & Warm* \\
\hline \multirow{2}{*}{ Wild* } & & & Wild* \\
\hline & Worried* & Worried* & Worried* \\
\hline
\end{tabular}

Emotion terms also present in ESP 


\section{Emotion Profiles for Clusters of Coffee Users}

Principle component analyses were conducted on each consumer cluster, examining how the 44 emotions related to coffee drinking portray and represent different coffee samples.

\section{Consumer cluster 1: users who like all coffee samples}

While this cluster of consumers liked all samples equally, the emotion profiles underlining each sample were distinct (Figure 3.2). Positive-high energy emotions were generated when drinking Sumatra (active, boosted, energetic, rested, empowering) and Italian (social, special, jump start - using PCs1 and 3). On the contrary, positive -low energy feelings were felt with Breakfast (comfortable, pleasant, warm) and Nantucket (relaxed, curious). Psychologists have identified these high and low energy feelings as one key dimension of human emotions. This dimension is often referred to as high-low level of engagement, or high-low arousal dimension. Another major dimension is described as the positive-negative emotions. These two dimensions are said to overlap and the high-low or positive-negative are not necessarily in the opposite directions on the map (Mayer \& Gaschke, 1988; Plutchick, 1980 \& 2001; Russell, 1980 \& 1989; Thayer, 1978 \&1989; Watson \& Tellegen, 1985). These emotion dimensions were also observed in the rest of the consumer clusters as well.

This consumer cluster felt pleased, good, merry, understanding, and annoyed when drinking Kona. Because this consumer cluster did not appear to have a clear preference on one coffee over another, the emotion profiles for each sample was a mixture of both positive and negative emotions. 


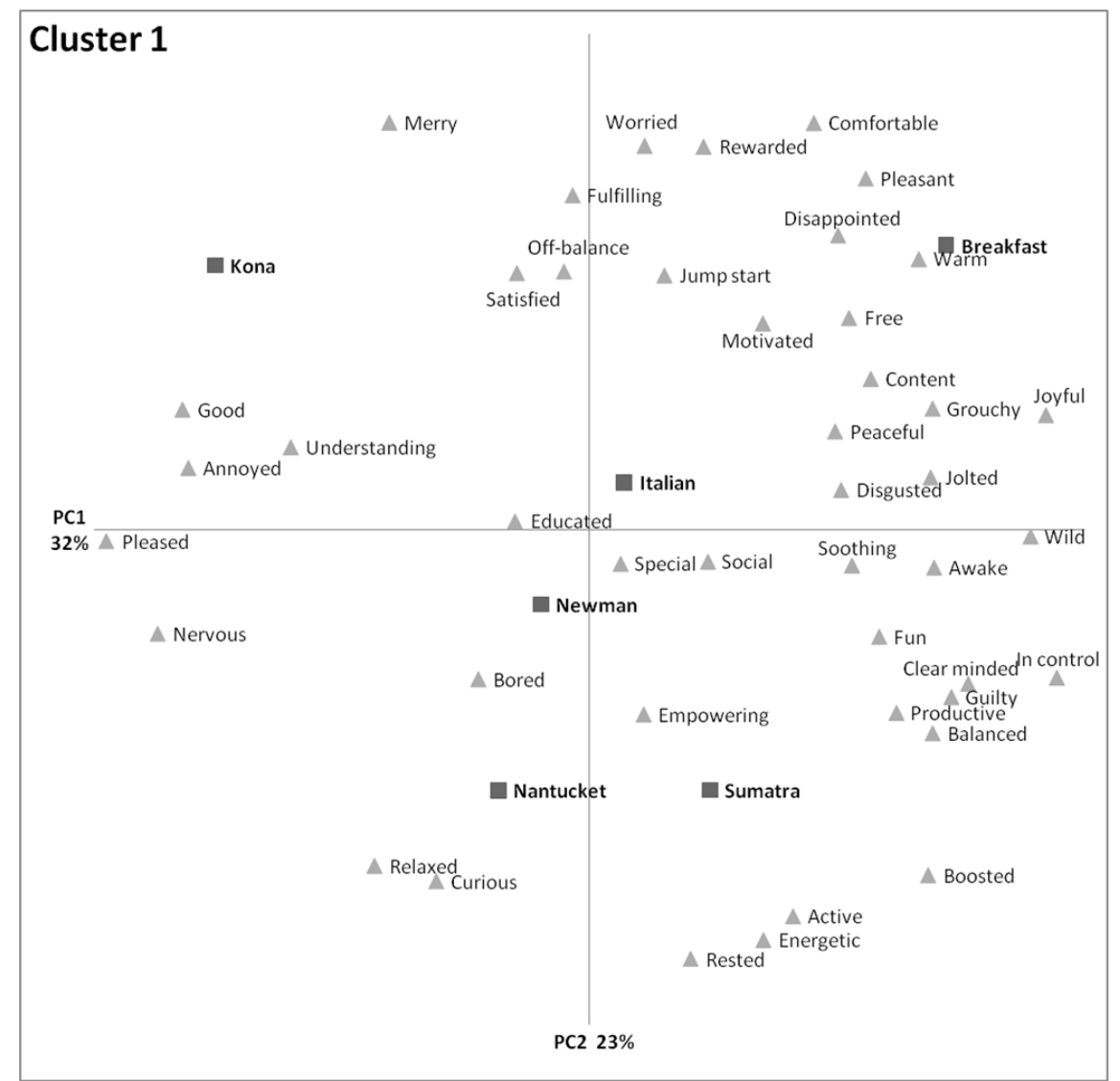

Figure 3.2. Coffee Drinking Experience (CDE) emotion profile for Cluster 1.

\section{Consumer cluster 2: users who disliked Breakfast}

Breakfast was anchored by disappointed, disgusted, bored, and annoyed. This was expected because it received the lowest liking score. The remaining samples were on the right quadrants, and were explained mostly by positive emotion terms consistent with the liking scores (Figure 3.3). Emotions elicited by Newman and Italian (merry, comfortable, soothing, content, relaxed, and clear-minded. Aside from clear-minded, the rest of the emotions seemed to be explained by the same basic dimension (contentment - a dimension in the hierarchical model of consumer emotions), which is supported by their similar liking score. According to the hierarchical model of consumer emotions (Laros \& Skeenkemp, 2005), emotions are classified into 4 positive dimensions (contentment, happiness, love, and pride) and 4 dimensions (anger, fear, sadness, and shame). However, Kona was shown to evoke peaceful, which falls into the contentment dimension of the hierarchical model, but was positioned at a different location on the bi-plot from Newman and Italian. On the other hand, the Consumption Emotion Set 
(Richins, 1997) described 16 dimensions of emotions which classify peacefulness and contentment in separate clusters. This suggested that structure of emotions is highly complex and may need more than 8 dimensions to describe the experience. The authors also speculated that peaceful may convey different meaning to this consumer cluster and they may not relate peaceful emotion to the feeling of contentment. Individual's perception of emotion descriptor varies, which has long been a challenge in the development of standard assessment tool for affective experience (King \& Meiselman, 2009). Next, Sumatra was described by motivated and educated feelings and Nantucket might have stimulated the emotion guilty. Sumatra received the same liking scores as Newman, Italian, and Kona, but its emotion profile is different. This supported King \& Meiselman's (2009) finding that the similar acceptability rating does not correlate to similar emotion profiles, and vice versa. Nantucket and also received the middle rating score of 'like slightly', hence, its position in the middle of the emotion space.

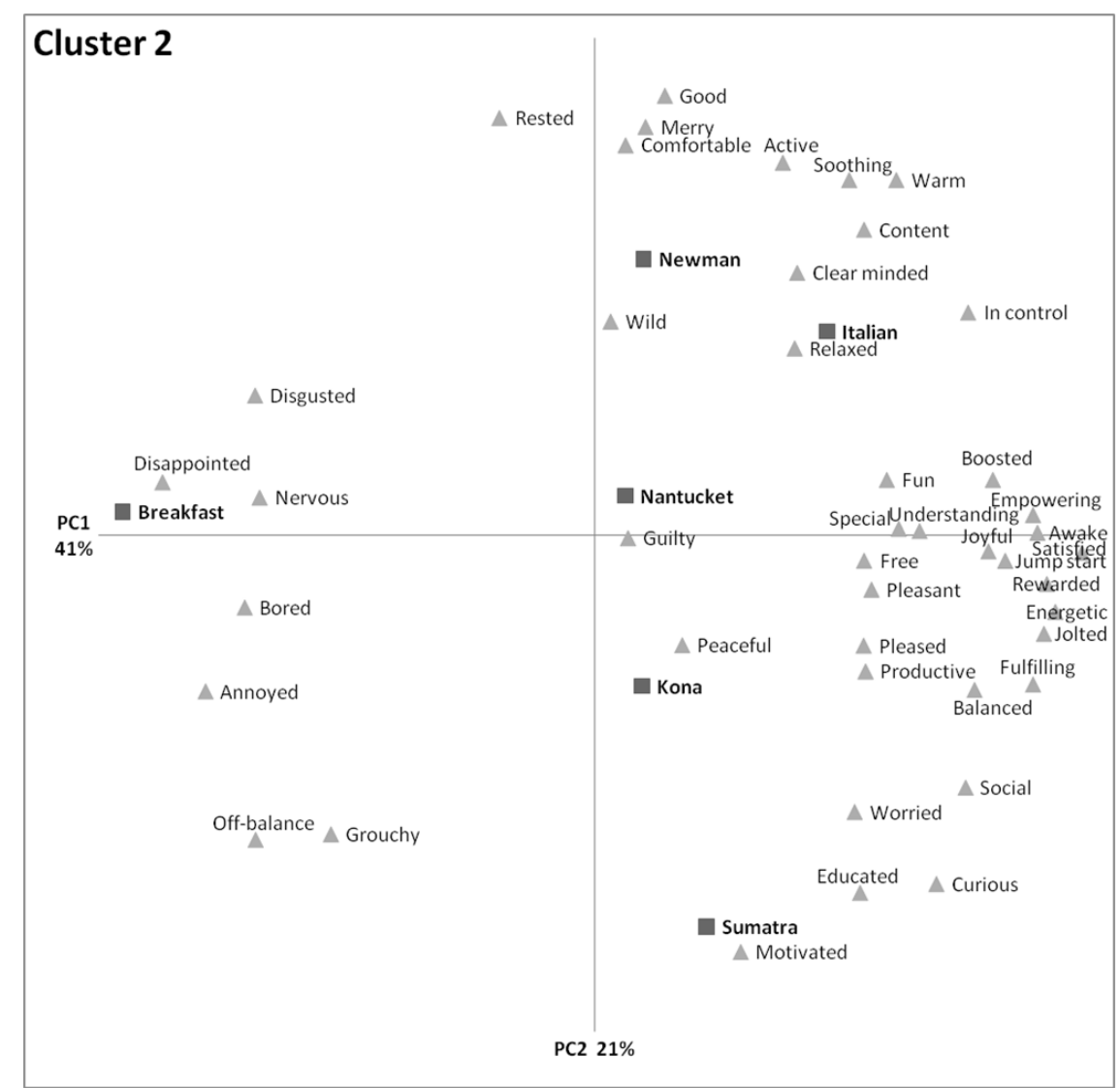

Figure 3.3. Coffee Drinking Experience (CDE) emotion profile for Cluster 2. 


\section{Consumer cluster 3: users who liked Breakfast and Nantucket}

Coffee drinkers belonging to this cluster gave Nantucket and Breakfast the highest liking scores, and Sumatra the lowest. PCs 1 and 3 were used to map the emotion profiles, and although PC 2 explained a higher percentage of data variation (20\%) than PC $3(18 \%)$, PC 3 was able to provide a clearer picture and more insight on the emotion profiles (Figure 3.4).

Nantucket stimulated positive emotion terms; comfortable, pleasant, satisfied, rewarded, special, and guilty, while Breakfast likely elicited similar emotions but at lower intensities. Italian and Kona received similar liking scores and their emotion profiles were comparable, explaining by the task-oriented emotion terms (Kona - clear-minded, social, motivated, active; Italian - merry, curious, productive). Consumers felt grouchy and wild when drinking Newman and were worried, disgusted, and disappointed when drinking Sumatra. It showed that this consumer cluster preferred Nantucket over coffees (Kona and Italian) that produced active, taskoriented emotions because Nantucket brought out positive-low energy emotions (Russell, 1980). These positive-low energy emotions are also classified under the 'contentment' and 'joy' dimensions in the CES (Richins, 1997) and under 'happiness' dimension in the hierarchical model (Laros \& Steenkemp, 2005).

Even though the liking scores of Italian, Kona, and Newman were not significantly different, their emotion profiles were distinct from one another. Again, this phenomenon was identified by King \& Meiselman (2009) that different emotion profiles do not always mean different liking scores. Also, this suggests that overall liking score is not an adequate measure of acceptability. It may be necessary to examine the sensory profiles of each to understand the sensory driver of grouchy emotion elicited by Newman which is necessary in the product development. 


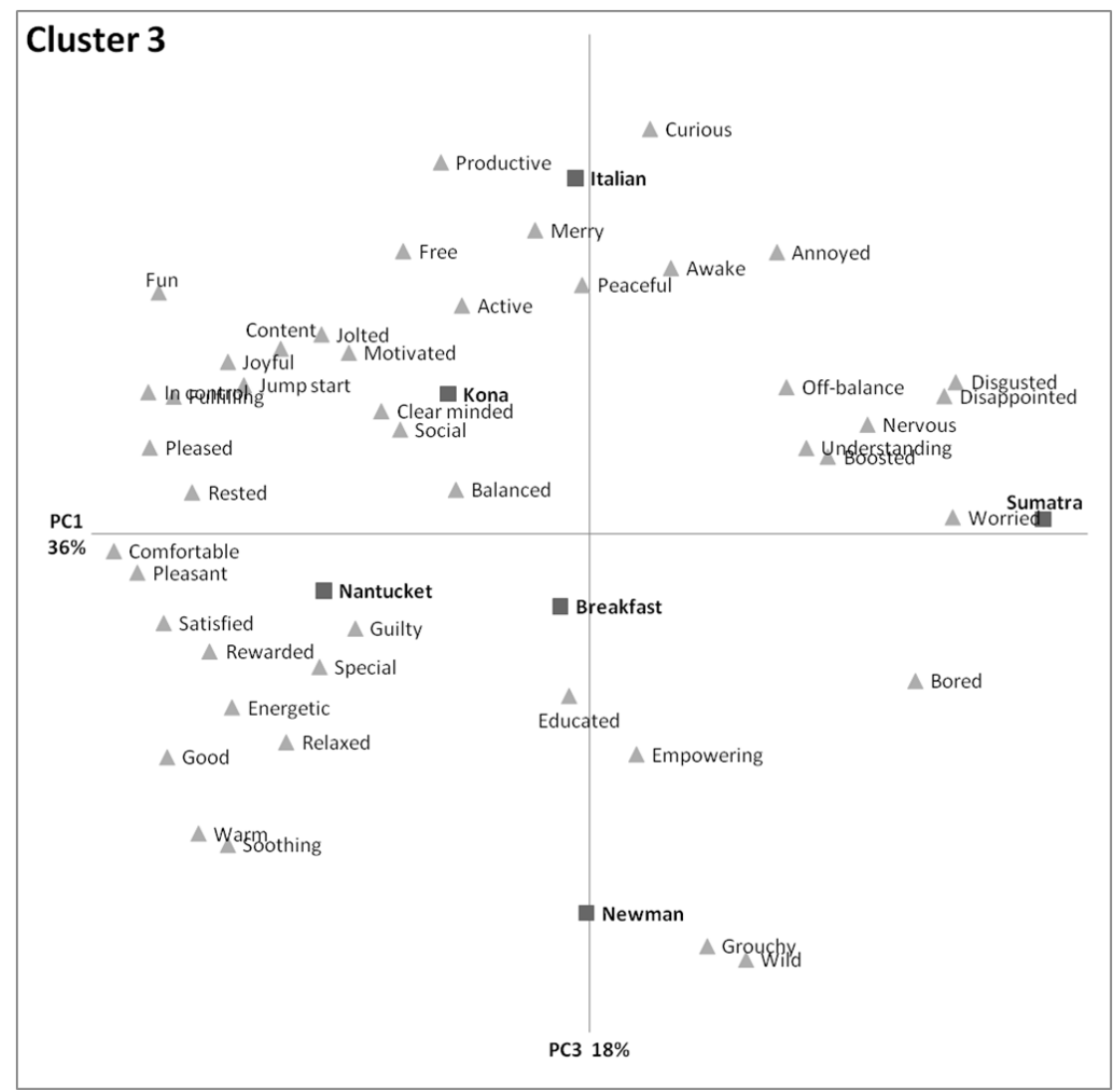

Figure 3.4. Coffee Drinking Experience (CDE) emotion profile for Cluster 3.

\section{Consumer cluster 4: users who did not give high scores to any coffee sample}

This consumer cluster did not appear to have strong positive or negative preferences for any sample, except for a dislike of Italian. Nantucket and Kona, receiving equally high rating, brought out positive emotions (fun, good, pleased, merry, and curious - Figure 3.5). Newman and Sumatra, scored in between 'like slightly' and 'neither like nor dislike', had mixed emotion profiles. Newman was explained by peaceful, grouchy, understanding, disappointed, and relaxed. Sumatra elicited a guilty feeling. Emotions related to food consumption are subtle and usually mixed. Therefore, it is not unexpected to see both positive and negative feelings describing a single product (Mano \& Oliver, 1993; Edell \& Burke, 1987), especially when the product received a middle-range liking score. Breakfast, although received lower rating than Newman and Sumatra (but not statistically significant), was described to stimulate the feeling of educated and some positive-high energy emotions (e.g., boosted, energetic, and empowering). This group of consumers disliked Italian and felt annoyed during the drinking experience. 
Because these consumers did not have a clear direction in their preferences, emotions elicited by each coffee were mixed with both positive and negative terms.

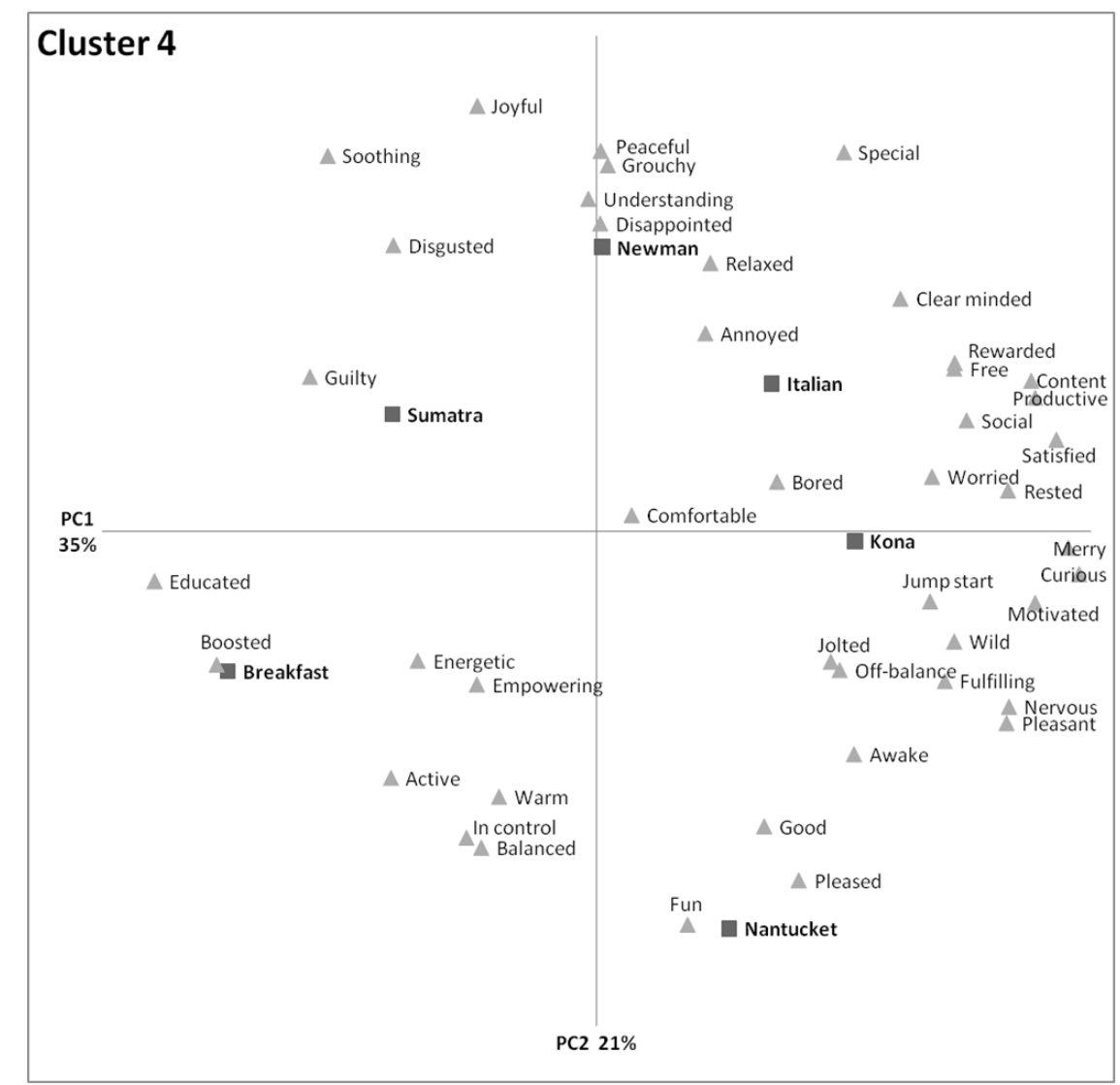

Figure 3.5. Coffee Drinking Experience (CDE) emotion profile for Cluster 4.

\section{Consumer cluster 5: users who liked Breakfast and disliked the rest}

Breakfast was the one sample liked by this cluster and brought out positive emotions, particularly empowering, educated, social, and peaceful (Figure 3.6). Nantucket (next best rating) also promoted positive feelings (clear-minded, guilty, soothing, understanding, curious). Kona (underlined by merry, joyful, and in-control) and Sumatra (underlined by worried) seemed to also be influenced by off-balance, wild, and jolted emotions that anchored the upper dimension of PC 2, where both were located. Newman and Italian were placed on the negative emotion dimension (the right quadrants) which included disgusted, disappointed, grouchy, annoyed, and bored. There is a trend that could be observed in this consumer cluster. The 
preference scores seemed to decrease as the roast level increased (degree of roasting from light to dark: Breakfast - Nantucket - Kona - Newman - Italian - Sumatra).

Although this cluster reported only liked Breakfast (light roast), the medium roasts (Nantucket and Kona) did not create negative emotions for them, only the darker roasts did (Newman, Italian, and Sumatra). This may be because the sensory characteristics of the medium roast coffees were more similar to the Breakfast coffee that they may be familiar with. Familiarity and product exposure lead to acceptability and consumption (Gibson, 2006).

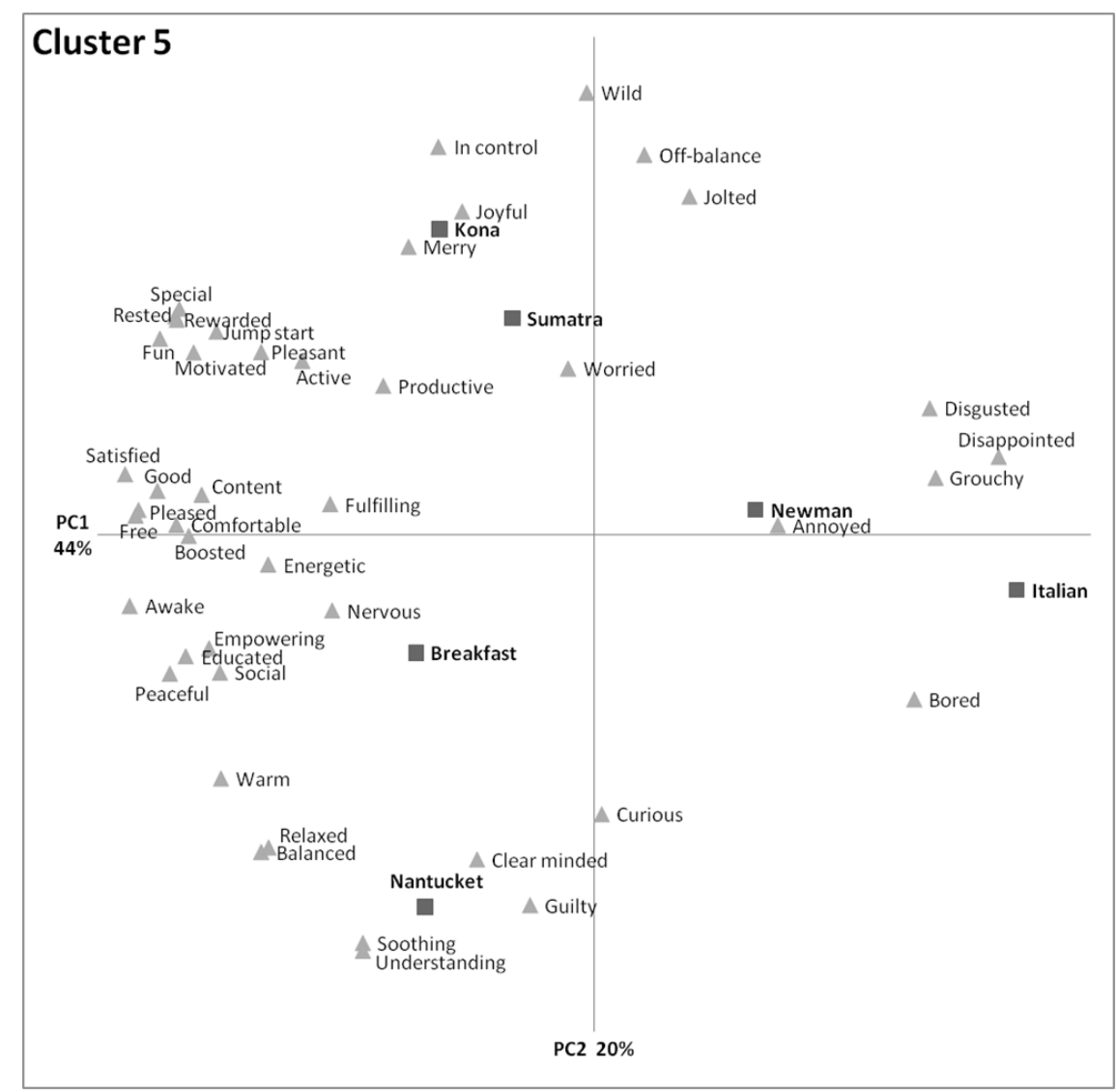

Figure 3.6. Coffee Drinking Experience (CDE) emotion profiles for Cluster 5.

\section{Consumer cluster 6: users who disliked Nantucket}

Kona was rated highest and Nantucket lowest for this consumer cluster, and they were situated on opposite ends of PC 1 (Figure 3.7). Kona was described by positive emotions that describe the mental state that is focused: motivated, clear-minded, balanced, productive, and empowering. Nantucket increased negative feelings, particularly worried and disappointed. 
Italian, Breakfast, and Sumatra received similar ratings of 'like slightly'. However, each seemed to be characterized by its own positive emotion profiles (Italian - relaxed; Sumatra - soothing and rewarded; Breakfast - special). Guilty and grouchy were elicited when drinking Newman. It seems that the most important aspect for this group of consumers is for coffee to offer them the task-oriented emotions over the positive-low energy feelings (Italian, Sumatra, and Breakfast). The sensory stimulation from Kona coffee appeared to accomplish this, hence the higher rating.

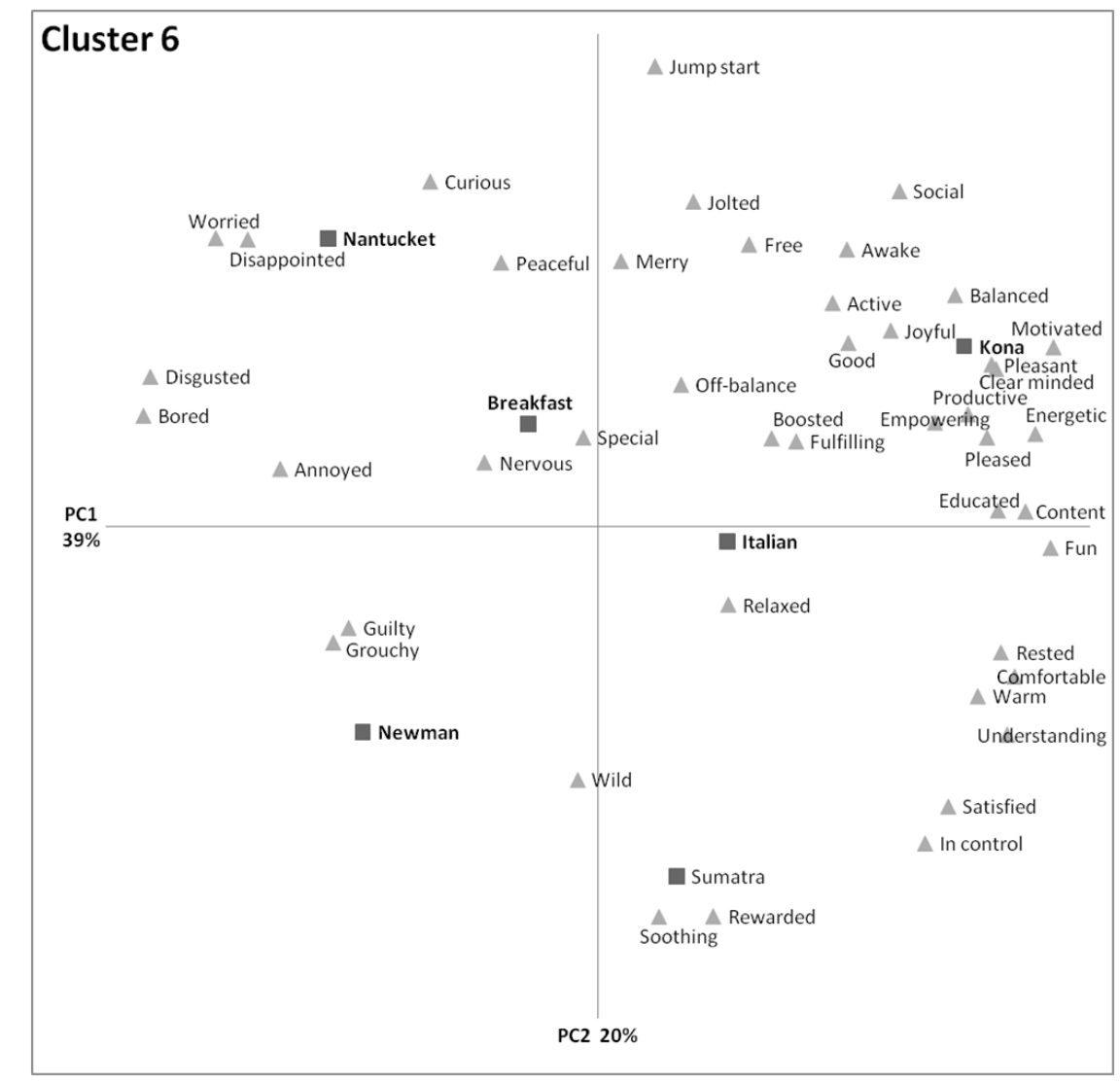

Figure 3.7. Coffee Drinking Experience (CDE) emotion profile for Cluster 6.

It is one of the conclusions that the each coffee sample generated different emotional responses for different consumer clusters. However, some consistencies were identified from this emotion study as follows.

The emotion terms derived from the coffee drinking experience seemed to be depicted by two main dimensions: positive-negative and high-low arousal. According to the PCA maps, these dimensions are not necessarily anchored on the opposite end of a linear axis and are likely 
interconnected, as also suggested by other researchers (Mayer \& Gaschke, 1988; Plutchik, 1980 $\& 2001)$.

In the majority of consumer clusters, Kona seemed to elicit emotions that describe a focused and task-oriented mental state (e.g., in-control, motivated, clear-minded). It may be useful to explore the sensory characteristics of Kona that are distinctive from other coffee samples.

This emotion study revealed additional group of emotions that describe the mental state that is active and task-oriented (e.g., clear-minded, motivated, productive) during coffee consumption. According to the psycho-evolutionary theorists, these emotions may be classified as high-arousal emotions since they stimulate adaptive responses to extrinsic stimuli to enable human to cope with stressors in daily life (Thayer, 1978; Izard 1977 \& 2009; Plutchik, 1980 \& 2001). To the authors' knowledge, these emotion states have not been identified in previous literatures relating to food product consumption. This research presents additional information specific to coffee drinking experience that could be useful for future researchers.

\section{Conclusion}

Stepwise regression analysis of the data yielded a core list of 44 CDE emotions that was able to illustrate defined distinctions among emotion responses from each coffee sample within the 6 consumer clusters. Of these 44 terms, 27 emotions were identified to be associated specifically with coffee drinking and 17 terms were more universal and applicable toward the general food consumption experience (from the ESP lexicon). The study also uncovered the additional group of emotions that describe the alert and focused mental state (e.g., motivated, incontrol, productive, clear-minded). These emotions were elicited by the coffee drinking experience and were not identified in the previously developed emotion scales.

Using these $44 \mathrm{CDE}$ emotions, the coffee drinking experience can be explained by two main dimensions: the positive-negative and the high-low energy dimensions. This study demonstrated that coffee drinkers not only had varying preferences for the coffees, but they also sought different emotion experiences from the beverage. Some preferred the drinking experience to elicit positive-lower energy feelings, some liked to be aroused by the positive-high energy emotions, and some desired for feelings of a focused mental state. 
This reduced CDE emotion list was shown to successfully characterize different coffee drinking experiences among the six clusters of 94 coffee drinkers. This list of emotions could be applied toward future emotion research with coffee users in different cultures and demographic profiles to identify the influences of these factors on the coffee perception. 


\section{References}

Bhumiratana, N., Adhikari, K., \& Chamber, E. IV. The development of emotion lexicon for the coffee drinking experience. Journal of Sensory Studies, Submitted.

Chrea, C., Grandjean, D., Delplanque, S., Cayeux, I., Le Calve, B., Aymard, L., Velazco, M.I., Sander, D., \& Scherer, K.R. (2009). Mapping the semantic space for the subjective experience of emotional responses to odors. Chemical Senses, 34, 49-62.

Edell, J.A., \& Burke, M.C. (1987). The power of feelings in understanding advertising effects. Journal of Consumer Research, 14, 421-433.

Gibson, E.L. (2006). Emotional influences on food choice: Sensory, physiological and psychological pathways. Physiology \& Behavior, 89, 53-61.

Hartel, C.E.J., and Hartel G.F. (2005). Cross-cultural differences in emotions: the why and how. Social Science Information, 44, 683-693.

Herz, R.S. (2005). Odor-associative learning and emotion: effects on perception and behavior. Chemical Senses, 30, i250-i251.

International Coffee Organization $@$ Coffee disappearance (consumption) data from 2000 to 2009. Available at http://www.ico.org/historical/2000+/PDF/CONSUMPTION.pdf> Accessed 10.10.

Izard, C.E. (1977). Human emotions. New York: Plenum Press.

Izard, C.E. (2009). Emotion theory and research: highlights, unanswered questions, and emerging issues. The Annual Review of Psychology, 60, 1-25

King, S.C., \& Meiselman, H.L. (2009) Development of a method to measure consumer emotions associated with foods. Food Quality and Preferences, 21, 168-177.

Labbe, D., Almiron-Roig, E., Hudry, J., Leathwood, P., Schifferstein, H.N.J., and Martin, N. (2009). Sensory basis of refreshing perception: role of psychophysiological factors and food experience. Physiology and Behavior, 98, 1-9.

Laros, F., \& Steenkemp, J. (2005). Emotion in consumer behavior: a hierarchical approach. Journal of Business Research, 58, 1437-1445.

Macht, M., Roth, S., \& Ellgring, H. (2002). Chocolate eating in healthy men during experimentally induced sadness and joy. Appetite, 39, 147-158.

Mano, H., \& Oliver, R.L. (1993). Assessing the dimensionality and structure of the consumption experience: Evaluation, feeling, and satisfaction. Journal of Consumer Research, 20, 451466. 
Mayer, J.D., and Gaschke, Y.N. (1988). The experience and meta-experience of mood. Journal of Personality and Social Psychology, 55, 102-111.

Meilgaard, M.C., Civille, G.V., \& Carr, B.T. (2007). Sensory Evaluation Techniques, Fourth Edition. Factors Influencing Sensory Verdict (pp. 39-43). Boca Raton, FL: CRC Press.

O’Mahony, M. (1986). Sensory evaluation of food - Statistical methods and procedures. Level of significance: type I and type II errors (pp. 66-68). New York: Marcel Dekker, Inc.

Plutchik, R. (1980). Emotions: A Psychoevolutionary Synthesis. New York: Harper \& Row.

Plutchik, R. (2001). The Nature of Emotions. American Scientist, 89, 344-350

Resurreccion, A.V.A. (1998). Consumer Sensory Testing for Product Development. Test Procedures (pp. 43-66). Gaithersburg, MD: An Aspen Publication.

Richins, M.L. (1997). Measuring emotions in the consumption experience. Journal of Consumer Research, 24, 127-146.

Russell, J.A. (1980). A circumplex model of affect. Journal of Personality and Social Psychology, 39, 1161-1178.

Russell, J.A. (1989). Affect grid: A single-item scale of pleasure and arousal. Journal of Personality and Social Psychology, 57, 493-502.

Russell, J.A. (1991). Culture and the categorization of emotions. Psychological Bulletin, 110, 426-450.

Scherer, K.R. (2005). What are emotions? And how can they be measured. Social Science Information, 44, 695-729.

Thayer, R.E. (1978). Toward a psychological theory of multidimensional activation (arousal). Motivation and Emotion, 2, 1-34

Thayer, R.E. (1989). The Biopsychology of Mood and Arousal. New York: Oxford University Press.

Thomson, D., Crocker, C., \& Marketo, C.G. (2010). Linking sensory characteristics to emotions: An example using dark chocolate.

Watson, D., and Tellegen, A. (1985). Toward a consensual structure of mood. Psychological Bulletin, 98, 219-235 


\section{CHAPTER 4 - Coffee Drinking and Emotions: Are There Key Sensory Drivers for Emotions?}

In the past decade or so, the coffee market has exploded, and to remain competitive in the market, it is important to identify the key drivers for consumer acceptance of coffee. The main purpose for coffee consumption is enjoyment and therefore, sensory and emotional experiences are the main measures of acceptability and consumption. This study expanded on the previous emotion study on the population of coffee drinkers in Manhattan, Kansas, USA and focused on identifying the sensory drivers of emotional responses assessed during the drinking experience. A highly-trained coffee panel performed descriptive analysis of the six coffee samples and identified the significant sensory attributes that discriminated each coffee. Utilizing Partial Least Square Regression, the sensory data were then mapped with the emotion data to determine the responsible sensory drivers for the eliciting emotional responses. It was found that the sensory characteristics of dark roast coffee (roast, burnt, bitter, and body/mouthfeel) may elicit positivehigh energy feelings for this population of coffee users. Tobacco (flavor/aroma) and cocoa (aroma) may also be responsible for positive emotions (content, good, and pleasant). Citrus, hay-like, and acidity seemed to be the negative sensory drivers as they induced the feeling of offbalance.

\section{Introduction}

Human senses are powerful elicitors of emotions and the interactions between the two are rarely debated (Chrea et al., 2009; Porcherot et al., 2010; Thomson, 2006). A number of studies have attempted to define and categorize human emotion, but only recently that emotions have been linked to food and beverage. Nowadays, there has been more awareness that the emotional experiences consumers received from a product via sensory perception determine acceptability and consumption (Thomson, Crocker, \& Marketo, 2010; Gibson, 2006). Therefore, the assessment of the emotional responses elicited by the sensorial experience during the product consumption is also vital. Several researchers developed emotion scales to measure the affective feelings evoked by the product consumption (EsSense Profile ${ }^{\mathrm{TM}}$; King \& Meiselman, 2009) or 
by olfactory stimulations from everyday odors (Geneva Emotion and Odor Scale; Chrea et al., 2009).

In Chaper 2, the emotions elicited by the coffee drinking experience were identified, as coffee is the second most consumed beverage and the U.S. is the largest coffee market in the world (International Coffee Organization $@$, 2009). They further determined a list of 44 emotions suitable for defining the drinking experiences and provided the emotion profiles for each segment of coffee drinkers. To have a complete understanding of consumers' perceptions, it is important to reveal the sensory characteristics that elicit those emotions experienced during coffee consumption.

Coffee is well known for complex sensory characteristics and is consumed mainly for the sensory experience it provides (Illy, 2002; Grosch, 1998; Czerny, Mayer, \& Grosch, 1999; Illy \& Viani, 2005). It is one of the few food products that has specialized experts or 'cuppers' to

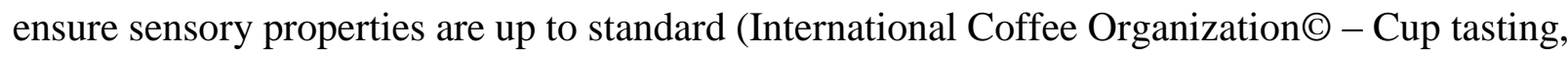
2010). Due to its high complexity, the descriptive sensory panel may need training specifically on coffee, in addition to the usual intensive training program on the sensory of food and beverage. Many studies supported the amount of training and regular re-training correlate with panelist perception of the sensory attributes and increased the quantification accuracy of attribute intensities (Chambers, Allison, \& Chambers, 2004; Chambers \& Smith 1993; Wolters \& Allchurch 1994; Bitnes, Ueland, Moller, \& Marten, 2008).

The main objective of this study was to identify sensory drivers of emotional response to the experience of coffee drinking. A specifically-trained coffee panel performed descriptive analysis on the coffee samples that were used to elicit emotions in coffee drinkers. The sensory data was then utilized to determine the sensory drivers for emotional responses in each segment (cluster) of consumers.

\section{Materials and Methods}

\section{Descriptive panel}

The descriptive coffee panel from the Wolf group (Cincinnati, OH, USA) was utilized to evaluate the coffee samples. The panel consisted of six highly-trained members who had completed $120 \mathrm{~h}$ of general training and had a minimum of 1,200 $\mathrm{h}$ of sensory testing of food 
and beverages. The coffee panelists also completed an additional $120 \mathrm{~h}$ of training on various coffee stimuli, key attributes (coffee, robusta, roasted, burnt, earthy, rioy, acidity, bitter, and body/mouthfeel), and references were used to anchor intensity scores for each key attribute. Performance of the panel is evaluated every 3 months in the form of a blind reference sample or samples. This coffee panel has been evaluating coffee products regularly for over 2 years before doing this study.

\section{Coffee Samples}

The six single-serve coffee samples (K-Cup® Keurig, Inc.; Reading, MA, USA) were evaluated by the two descriptive sensory panels. These single-serve coffee samples represented the range of roast levels from light to dark. Green Mountain Breakfast Blend represented the light roast. Green Mountain Nantucket Blend represented the blend of medium roasted African and Indonesian beans mixed with some French roast. Green Mountain Sumatra Reserved represented dark roasted organic Sumatra coffee. Tully's Kona represented the blend including the famous Hawaiian coffee from the Kona Typica varietal, and was classified as medium roast. Tully’s Italian Roast represented a blend of dark roast. Lastly, Newman’s Own Organic represented a blend of medium and dark roast organic coffee beans. All coffee samples were stored at room temperature $\left(20^{\circ} \mathrm{C}\right)$ until testing and were used in the study within six weeks of their delivery.

\section{Descriptive Sensory Analysis}

\section{Sample Preparation and Serving}

Keurig® Special Edition B60 Brewing System (Keurig®, Inc.; Reading, MA, USA) was used to brew the single-serve K-Cup® coffee samples. The machine was set up and cleaned following the instructions in the user's manual. A K-Cup was placed in the K-Cup holder and $157.5 \mathrm{~mL}$ of coffee was selected to brew into the cup. A ceramic mug (Econo Rim, Syracuse China; Lyncourt, NY, USA) was used for the expert panel and a styrofoam cup (Dart J-cup, Dart Container Corp.; Mason, OH, USA) was used for the coffee panel. The coffee cups were labeled with 3-digit random numbers prior to serving. After each brewing cycle was completed, the KCup was removed and discarded immediately. Each coffee cup was covered with either a saucer (Econo Rim, Syracuse China; Lyncourt, NY, USA), or a plastic lid (Dart Container Corp.; 
Mason, $\mathrm{OH}, \mathrm{USA}$ ), and was then served immediately to the panelists monadically in random order.

\section{Sample Evaluation}

One 180-min orientation session was completed to familiarize the descriptive panel with the samples. During orientation, the panel identified and defined aroma, flavor, and texture attributes present in each sample (Table 4.1). Necessary references were determined to anchor and calibrate the intensity measurement on a numerical 15 -pt scale with 0.5 increments $(0.0=$ none; $15.0=$ extremely high intensity).

Outlined in the following paragraph is the structured tasting protocol. Once the coffee was served, panelists opened the lid and the temperature of the coffee was taken with a digital thermometer (Model T220/38A Latte Thermometer, Comark; Hertfordshire, United Kingdom). When the temperature reached $65.5^{\circ} \mathrm{C}$, the lid was replaced, keeping one end slightly opened. The panelists took a sniff to identify aroma descriptors belonging to that particular coffee. Panelists then slurped the sample and gently manipulated it in the mouth for 10-20 s to evaluate flavor and body/mouthfeel attributes. At $60.5-63{ }^{\circ} \mathrm{C}$, panelists tasted the sample again, as this temperature was ideal to evaluate acidity and bitter attributes. A small amount of sample was swallowed to discern bitterness on the back of the tongue. Afterward samples were expectorated. A 10-min break was taken between each sample, during which buttered bread and distilled water were used as palate cleansers. Buttered bread was prepared by spreading Land O'Lakes Whipped Butter (Whipped Butter Sweet Cream, Salted, 45\% less fat, Land O'Lakes, Inc.; Arden Hills, MN, USA) on a $3 / 4$ cm slice of European Bataard bread (Kroger; Cincinnati, OH, USA).

During testing, panelists evaluated a total of four samples per 180-min panel session. Samples were served one at a time, and tasted individually by each panelist. Then a group discussion was initiated by a panel leader to determine attributes present, their strengths, and identify which references were needed. A new cup of the same sample was then served, along with references. The panel then individually evaluated the sample on ballots. The ratings were collected and written on the board by the panel leader. This was to identify any problem areas and whether other references should be reviewed. The panelists then determined and recorded their final score for the first replication of the sample. The next sample was served after a 10min break, and was evaluated following the same procedure. 
Table 4.1. The list of aroma, flavor, and texture descriptors identified by the coffee panel

\begin{tabular}{|c|c|}
\hline Attributes & Definitions \\
\hline Coffee & Amount or strength of Arabica coffee aroma or flavor \\
\hline Robusta & $\begin{array}{l}\text { A rubbery-like flavor character typical of Robusta coffees; is sometimes present in lower quality coffees (e.g., burnt rubber tire, } \\
\text { Robusta coffee beans) }\end{array}$ \\
\hline Roast & Degree to which the coffee is roasted; ranges from green/no roast - low - medium - dark - very dark \\
\hline Burnt & Aromatics associated with blacked/acrid carbohydrates (e.g., burnt toast, espresso coffee) \\
\hline Earthy & An earthy/dirty aromatic similar to wet soil or potato skins (e.g., wet potting soil) \\
\hline Rioy & Aromatic associated with iodine in water; is described as chlorine-like, brassy, metallic, and chemical \\
\hline Ashy & Bark-like lingering aromatics associated with a cold campfire \\
\hline Acidity & A sour, sharp, puckering sensation in the mouth caused by acids \\
\hline Tobacco & Characteristic reminiscent of tobacco's odor and taste, but should not be used for burnt tobacco \\
\hline Stale & Not fresh, flat, bodied down or reduced; old \\
\hline Hay-like & Slightly sweet dry, dusty aromatics with a slight green character associated with dry plant material \\
\hline Citrus & Aromatics associated with citrus fruits (e.g., lemon) \\
\hline Cocoa & Brown, sweet, dusty often biter aromatics associated with cocoa beans and powered cocoa \\
\hline Sweet Identity & Aromatic associated with the impression of sweet products \\
\hline Floral & Sweet, light, slightly perfumy impression associated with flowers \\
\hline Bitter & The amount of bitter basic taste; (e.g., caffeine solutions) \\
\hline Body/mouthfeel & Viscosity of the coffee; heaviness on the tongue: thin - thick \\
\hline
\end{tabular}




\section{Emotion Data}

The emotion profiles each of the six coffee samples created for 94 coffee drinkers were determined through development and application of a scale of emotions related to the coffee drinking experience. Overall liking and intensity of emotion elicited by the coffee drinking experience were measured. Consumers were clustered into 6 clusters based on their coffee liking scores. Emotion profiles for each coffee were generated for each consumer cluster (Chapter 3)

\section{Statistical Analyses}

Randomized complete block design was used for the descriptive evaluation of the six coffee samples. Analysis of Variance using GLIMMIX procedure at 5\% level of significance (SAS® system version 9.2; SAS institute; Cary, NC, USA) was performed on the data set to determine attributes significant in identifying differences among products. Coffee sample was the fix effect. Panelist was set as a random effect. Once the set of significant descriptors was established, principal component analysis (Unscrambler® Camo Software A/S, Oslo, Norway) was performed on the significant sensory descriptors to inspect the sensory profile of each coffee sample.

To investigate the relationship between the sensory attributes and the emotional responses to the drinking experience, partial least squares repression (PLSR, Unscrambler ${ }^{\circledR}$ ) was conducted. Sensory drivers associated with the emotional experiences were identified among the 94 coffee users and in each consumer cluster.

\section{Results and Discussion}

\section{Descriptive Sensory Data}

The sensory elements detected as significantly different among the six coffee samples (pvalue $<0.05$ ) are illustrated in Table 4.2. 
Table 4.2. The sensory attributes detected by the coffee panel to significantly differentiate the six coffees.

\begin{tabular}{|c|c|c|c|c|c|c|}
\hline AROMA & BREAKFAST & ITALIAN & KONA & NANTUCKET & NEWMAN & SUMATRA \\
\hline Coffee & $7.79^{\mathrm{ab}}$ & $5.42^{\mathrm{c}}$ & $8.33^{\mathrm{a}}$ & $8.58^{\mathrm{a}}$ & $7.50^{\mathrm{ab}}$ & $8.58^{\mathrm{a}}$ \\
\hline Roast & $6.92^{\mathrm{b}}$ & $8.50^{\mathrm{a}}$ & $7.42^{\mathrm{b}}$ & $8.50^{\mathrm{a}}$ & $7.58^{\mathrm{b}}$ & $8.58^{\mathrm{a}}$ \\
\hline Burnt & $0.67^{\mathrm{c}}$ & $4.50^{\mathrm{a}}$ & $0.17^{\mathrm{c}}$ & $2.92^{\mathrm{b}}$ & $2.83^{\mathrm{b}}$ & $4.33^{\mathrm{a}}$ \\
\hline Rioy & $0.00^{\mathrm{b}}$ & $0.00^{\mathrm{b}}$ & $0.00^{\mathrm{b}}$ & $1.33^{\mathrm{a}}$ & $1.58^{\mathrm{a}}$ & $1.58^{\mathrm{a}}$ \\
\hline Ashy & $0.00^{\mathrm{b}}$ & $0.00^{\mathrm{b}}$ & $0.00^{\mathrm{b}}$ & $2.75^{\mathrm{a}}$ & $0.00^{\mathrm{b}}$ & $1.92^{\mathrm{a}}$ \\
\hline Hay-like & $2.67^{\mathrm{a}}$ & $0.00^{\mathrm{b}}$ & $0.00^{\mathrm{b}}$ & $0.00^{\mathrm{b}}$ & $0.00^{\mathrm{b}}$ & $0.00^{\mathrm{b}}$ \\
\hline Citrus & $2.00^{\mathrm{a}}$ & $0.00^{\mathrm{b}}$ & $0.00^{\mathrm{b}}$ & $0.00^{\mathrm{b}}$ & $0.00^{\mathrm{b}}$ & $0.00^{\mathrm{b}}$ \\
\hline Floral* & $0.00^{\mathrm{b}}$ & $0.00^{\mathrm{b}}$ & $0.00^{\mathrm{b}}$ & $0.00^{\mathrm{b}}$ & $0.00^{\mathrm{b}}$ & $1.08^{\mathrm{a}}$ \\
\hline Sweet Identity* & $0.00^{\mathrm{b}}$ & $1.00^{\mathrm{a}}$ & $0.00^{\mathrm{b}}$ & $0.00^{\mathrm{b}}$ & $0.00^{\mathrm{b}}$ & $0.00^{\mathrm{b}}$ \\
\hline Tobacco & $0.00^{\mathrm{b}}$ & $7.75^{\mathrm{a}}$ & $0.00^{\mathrm{b}}$ & $0.00^{\mathrm{b}}$ & $0.00^{\mathrm{b}}$ & $0.00^{\mathrm{b}}$ \\
\hline Cocoa & $0.00^{\mathrm{b}}$ & $0.00^{\mathrm{b}}$ & $2.33^{\mathrm{a}}$ & $0.00^{\mathrm{b}}$ & $0.00^{\mathrm{b}}$ & $0.00^{\mathrm{b}}$ \\
\hline Stale & $0.00^{\mathrm{b}}$ & $0.00^{\mathrm{b}}$ & $0.00^{\mathrm{b}}$ & $0.00^{\mathrm{b}}$ & $3.08^{\mathrm{a}}$ & $0.00^{\mathrm{b}}$ \\
\hline \multicolumn{7}{|l|}{ FLAVOR } \\
\hline Coffee & $8.25^{\mathrm{c}}$ & $8.00^{\mathrm{c}}$ & $11.75^{\mathrm{a}}$ & $10.33^{\mathrm{b}}$ & $11.75^{\mathrm{a}}$ & $12.50^{\mathrm{a}}$ \\
\hline Roast & $7.08^{c}$ & $10.17^{\mathrm{a}}$ & $8.54^{\mathrm{b}}$ & $8.92^{\mathrm{b}}$ & $10.50^{\mathrm{a}}$ & $10.08^{\mathrm{a}}$ \\
\hline Burnt & $1.58^{\mathrm{d}}$ & $6.67^{\mathrm{b}}$ & $6.75^{\mathrm{b}}$ & $3.75^{\mathrm{c}}$ & $8.33^{\mathrm{a}}$ & $8.50^{\mathrm{a}}$ \\
\hline Rioy & $0.00^{\mathrm{b}}$ & $0.00^{\mathrm{b}}$ & $0.00^{\mathrm{b}}$ & $2.04^{\mathrm{a}}$ & $2.00^{\mathrm{a}}$ & $1.58^{\mathrm{a}}$ \\
\hline Ashy & $0.00^{\mathrm{b}}$ & $0.00^{\mathrm{b}}$ & $0.00^{\mathrm{b}}$ & $2.92^{\mathrm{a}}$ & $0.00^{\mathrm{b}}$ & $2.83^{\mathrm{a}}$ \\
\hline Hay-like & $2.00^{\mathrm{a}}$ & $0.00^{\mathrm{b}}$ & $0.00^{\mathrm{b}}$ & $0.00^{\mathrm{b}}$ & $0.00^{\mathrm{b}}$ & $0.00^{\mathrm{b}}$ \\
\hline Citrus & $4.42^{\mathrm{a}}$ & $0.00^{\mathrm{b}}$ & $0.00^{\mathrm{b}}$ & $0.00^{\mathrm{b}}$ & $0.00^{\mathrm{b}}$ & $0.00^{\mathrm{b}}$ \\
\hline Floral* & $0.00^{\mathrm{b}}$ & $0.00^{\mathrm{b}}$ & $0.00^{\mathrm{b}}$ & $0.00^{\mathrm{b}}$ & $0.00^{\mathrm{b}}$ & $0.17^{\mathrm{a}}$ \\
\hline Tobacco & $0.00^{\mathrm{b}}$ & $8.08^{\mathrm{a}}$ & $0.00^{\mathrm{b}}$ & $0.00^{\mathrm{b}}$ & $0.00^{\mathrm{b}}$ & $0.00^{\mathrm{b}}$ \\
\hline Cocoa* & $0.00^{\mathrm{b}}$ & $0.00^{\mathrm{b}}$ & $0.67^{\mathrm{a}}$ & $0.00^{\mathrm{b}}$ & $0.00^{\mathrm{b}}$ & $0.00^{\mathrm{b}}$ \\
\hline Stale & $0.00^{\mathrm{b}}$ & $0.00^{\mathrm{b}}$ & $0.00^{\mathrm{b}}$ & $0.00^{\mathrm{b}}$ & $4.42^{\mathrm{a}}$ & $0.00^{\mathrm{b}}$ \\
\hline Acidity & $5.92^{\mathrm{a}}$ & $4.83^{\mathrm{c}}$ & $5.83 \mathrm{a}^{\mathrm{b}}$ & $5.92^{\mathrm{a}}$ & $4.92^{c}$ & $4.92^{c}$ \\
\hline Bitter & $3.08^{\mathrm{d}}$ & $9.50^{\mathrm{a}}$ & $8.13^{\mathrm{b}}$ & $5.42^{\mathrm{c}}$ & $8.00^{\mathrm{b}}$ & $8.42^{\mathrm{b}}$ \\
\hline \multicolumn{7}{|l|}{ TEXTURE } \\
\hline Body/mouthfeel & $6.38^{\mathrm{d}}$ & $8.67^{\mathrm{ab}}$ & $8.33^{\mathrm{b}}$ & $7.63^{c}$ & $9.13^{\mathrm{a}}$ & $8.83^{\mathrm{ab}}$ \\
\hline
\end{tabular}


The coffee descriptive panel differentiated sensory elements that were distinctive to each coffee sample. Ashy was identified in Nantucket and Sumatra, and was perceived to be more intense in Nantucket (medium roast). Rioy was detected at the same intensity level in Nantucket, Newman, and Sumatra, but was not present in the other samples. Tobacco only appeared in the Italian sample, stale underlined Newman, and cocoa aroma was unique to Kona. The attributes floral, sweet identity, and cocoa aroma were perceived at very low intensity $(\leq 1)$, and were later removed from the data set. Even after these attributes were eliminated the sensory fingerprint for each coffee still remained.

Principle component analysis was performed to visualize the product placements on the sensory space based on the significant attributes. Figure 4.1 illustrates sensory profiles of the coffees created by the coffee panel.

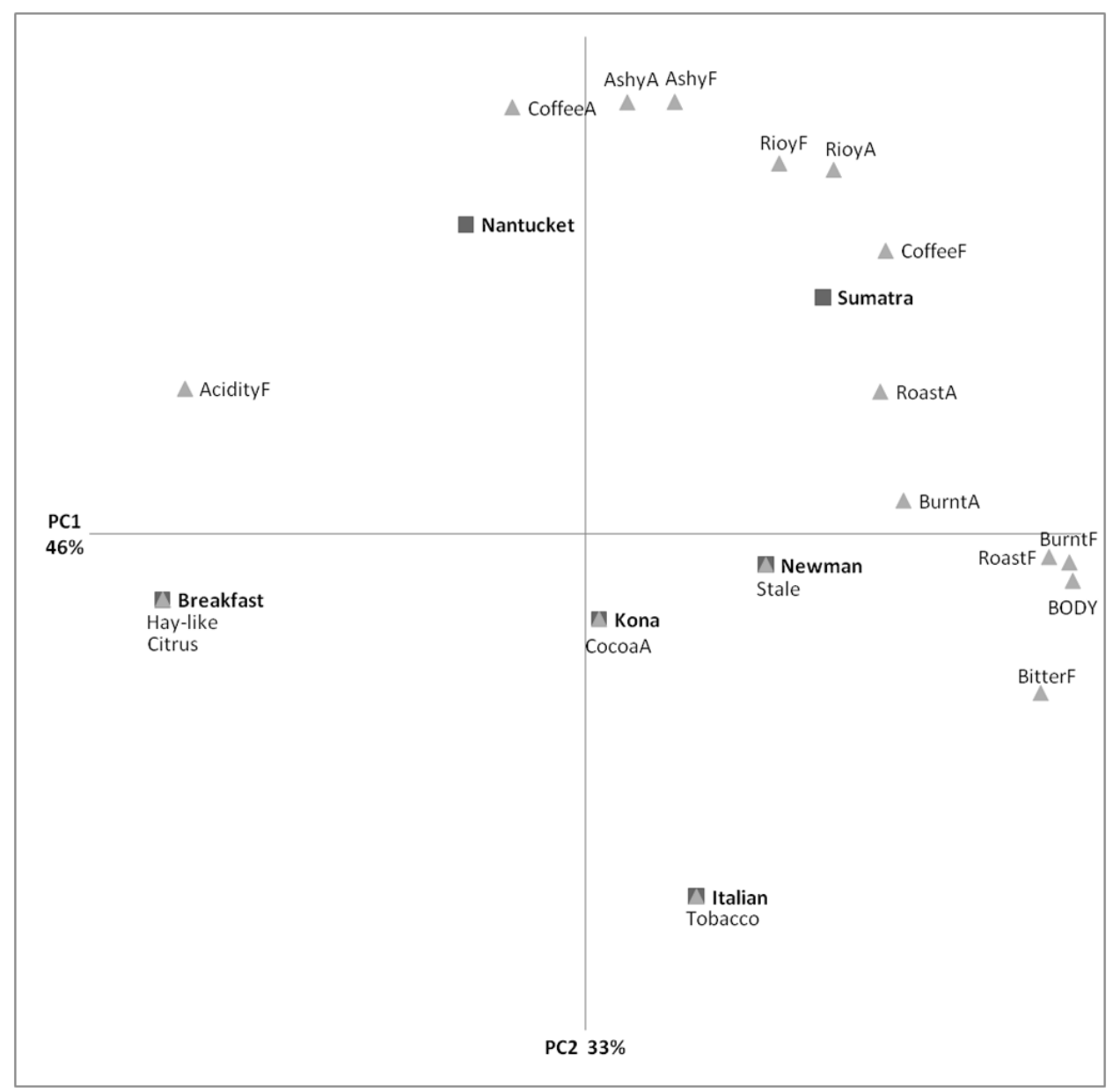

Figure 4.1. Sensory profiles of the six coffees generated by the coffee panel. 
PC1 explained $46 \%$ of the data variation and seemed to reflect characteristics generated by roasting. Acidity, hay-like, and citrus anchored one end of PC1 and described Breakfast. Burnt, roast, bitter, and body/mouthfeel anchored the other direction of PC1, and characterized Newman and Sumatra. Acidity, bitter, burnt (flavor and aroma), roast flavor, coffee flavor (except for Italian) and body/mouthfeel were influenced by degree of roasting. Acidity was more intense in the lighter roasts, while bitter, burnt (flavor and aroma), roast and coffee flavors, and body/mouthfeel increased with degree of roasting. The impact of degree of roasting on aroma and flavor in coffee has been extensively verified (Sivetz \& Desrosier, 1979; Schenker et al., 2002; Yeretzian, Jordan, Badoud, \& Lindinger, 2002; Illy and Viani, 2005; Baggenstoss, Poisson, Kaegi, Perren, \& Escher, 2008; Bhumiratana, Adhikari, \& Chambers, submitted-2010) and is supported by this research. However, degree of roasting was not the only factor affecting the sensory characteristics of coffee. PC2 explained 33\% of the data set and provided additional information on sensory elements for Nantucket, Kona, and Italian. Coffee aroma and roast aroma did not seem to be dependent on roast level. The intensities of these aroma attributes for Nantucket (medium roast) were higher than Newman (medium-dark roast), and were as high as Sumatra (dark roast). The sensory profiles indicated some sensory attributes were independent of degree of roasting, which confirmed that other factors may be influencing the sensory characteristics of coffee. The origins of coffee, including growing regions and variety of bean, evidently have noticeable impact on the sensory fingerprint of each coffee; this is supported by numerous studies (Mayer, Czerny, \& Grosch, 1999; Decazy, Avelino, Guyot, Perriot, Pineda, \& Cilas, 2003; Illy and Viani, 2005; Nebesny and Budryn, 2006; Ross, Pecka, \& Weller, 2006; Bhumiratana et al., submitted-2010).

The sensory from the descriptive panel was then utilized in the next step to identify the sensory drivers responsible for the emotional responses elicited by the coffee drinking experience.

\section{Identifying Sensory Drivers for the Emotional Experience}

The sensory descriptive data was studied with emotion responses of the same set of coffee samples created by 94 coffee drinkers in the study done in Chapter 3. Partial Least Square Regression (PLSR) was utilized to identify some sensory drivers of the emotion responses (Figure 4.2). Coffee aroma, surprisingly, elicited a range of negative emotions (bored, disgusted, annoyed, and disappointed) even though it is well-known that 'coffee aroma' elicited positive 
feelings, including alertness of the mental state, and is the driver of coffee consumption (Illy \& Viani, 2005; Seo, Hirano, Shibato, Rakwal, Hwang, \& Masuo, 2008). This may be because the definition of coffee aroma used by the coffee panel and consumers could be different, a common problem in the field of consumer research when integrating sensory and consumer data together. Coffee aroma, by the definition listed in Table 4.1, was the aroma of pure Arabica beans, which consumers may not be familiar with, might have led to a negative perception (Gibson, 2006).

Positive emotions seemed to be driven by cocoa aroma, bitter, tobacco, roast, burnt, and body/mouthfeel. Cocoa aroma may elevate good and pleasant emotions, which was consistent with previous studies. King \& Meiselman (2009) found that among the five food categories evaluated, chocolate was reported to have the highest ratings for 15 of the positive emotions (out of 24 positive emotions on a list of 39 terms). Macht \& Mueller (2007) reported consumption of chocolate to immediately reduce negative mood state, although the effect was temporary. It is also a common knowledge that chocolate and its resemblance usually induce positive feelings in a general population. Tobacco (flavor and aroma) evoked the feelings of jolted and content. Coffee users may initially be surprised (i.e., jolted) by the unfamiliar tobacco attribute that was not commonly found in all coffee (only one coffee sample in this study exhibited this sensory attribute). However, they were accepting of the experience (i.e., content), which indicate that having a tobacco attribute in coffee could potentially enhance the drinking experience for general coffee users. Bitter aroused energetic and productive feelings. Roast and burnt (flavor and aroma), and body texture made consumers feel jump start, satisfied, boosted, and special. On the contrary, citrus, hay-like, and acidity appeared to elicit off-balance feeling. Similar to tobacco, consumers may not be familiar with experiencing these sensory characteristics in coffee and were caught off-guard by them. Unlike tobacco, they may not find these attributes appropriate for coffee, hence the off-balance emotion. Because emotions are context specific (Richins, 1997), it seems that citrus, hay-like, and acidity attributes may not fit well with the concept of coffee, which caused negative feeling to develop.. 


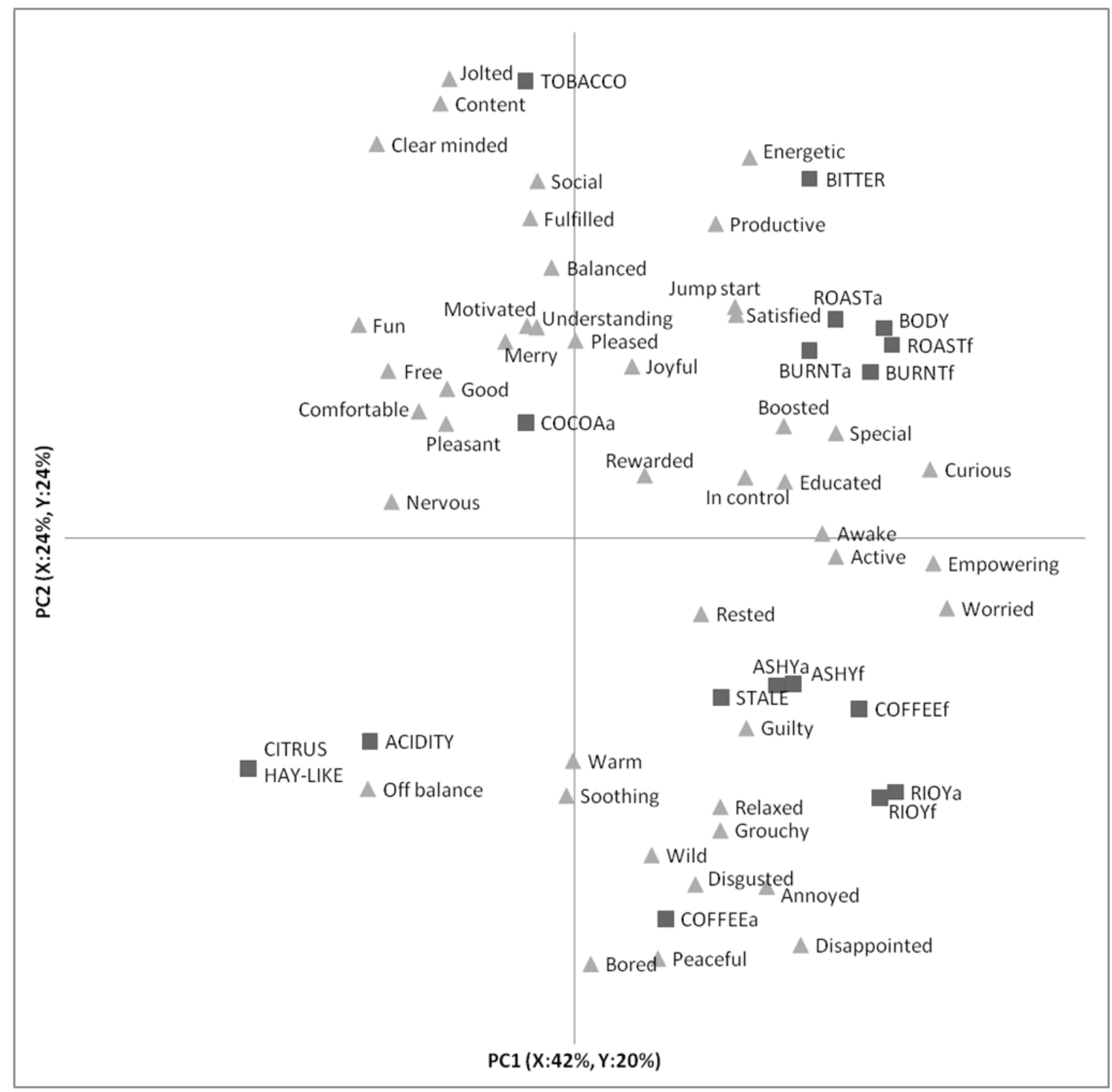

Figure 4.2. Partial Least Square Regression Analysis of Descriptive Sensory and Emotion Data of the Six Coffee Samples for 94 Consumers 
It seems the characteristics of dark roast coffee (roasted, burnt, bitter, and body/mouthfeel) elicited positive-high energy feelings. This is likely because there were more participants who preferred darker roasts since coffee preference was not one of the criteria during recruitment. Tobacco also induced feeling of contentment. This finding identified tobacco, roasted, burnt, bitter, and body/mouthfeel as the sensory drivers for this population of 94 coffee users.

Since consumers have varying preferences and are affected differently by sensory stimuli, the 94 coffee users were examined more closely in Chapter 3. The entire set of drinkers was clustered into six groups based on their coffee preferences and generated emotion profiles for each set of consumers. In this study, we conducted a PLSR analysis again on each consumer cluster to determine whether relationships can be drawn between the sensory characteristics and emotions elicited by the perceived attributes for each consumer cluster.

For coffee drinkers in Cluster 1 who liked all the coffees (Figure 4.3), tobacco attribute seemed to elicit social, jump start, and special feelings, while the characteristics of dark roasts (high intensity of roast, burnt, and body/mouthfeel) appeared to make them feel empowering and relaxed. Acidity was associated with awake and disgusted, and may be a negative attribute for this group. Cluster 2 (Figure 4.4) consisted of consumers who dislike Breakfast (classified as light roast). The PLSR map indicated that attributes citrus, hay-like, and acidity elicited negative emotions (e.g., disappointed, disgusted, annoyed), and dark roast characteristics (roast, burnt, bitter, and body) were driving positive emotions (e.g., satisfied, energetic, rewarded, boosted, in control, empowering). This group of coffee drinkers was depicted to relate coffee aroma to grouchy emotion and tobacco attribute to clear-minded, wild, and good feelings. Cluster 3 was identified to like Nantucket and Breakfast but dislike Sumatra (Figure 4.5). The PLSR bi-plot illustrated that hay-like, citrus, and acidity brought out positive emotions (e.g., merry, pleasant, understanding, relaxed, rewarded) for this group of coffee drinkers. Empowering and boosted emotions seemed to be induced by coffee flavor, ashy, and rioy, while tobacco elicited feelings of off-balance, jolted, and social. Negative emotions (disappointed and disgusted) were driven by roast, burnt, and body characteristics. Coffee drinkers grouped into Cluster 4 were those that did not have preference for any of the six coffees. Because they did not have concrete preferences, the coffees may have elicited mixed emotions for this group. This is shown in the PLSR bi-plot for this cluster (Figure 4.6). Cluster 5 composed coffee drinkers who gave high 
liking rating to Breakfast and disliked the dark roasts (Newman, Italian, and Sumatra). Hay-like, citrus, and acidity were shown to explain positive emotions (e.g., relaxed, soothing, understanding, peaceful), and coffee aroma explained fun, rewarded, and pleased (Figure 4.7).

On the other hand, coffee flavor and rioy appeared to describe negative emotions, including nervous, disgusted, and annoyed. Coffee drinkers in Cluster 6 were classified as preferring Kona coffee. The PLSR bi-plot (Figure 4.8) reflects that this group of consumers were attracted to the cocoa aroma as most positive emotions (i.e., balanced, productive, fulfilled, awake, motivated, and energetic) were described by cocoa aroma, including. Tobacco also described good and soothing emotions, while acidity seemed to generate mixed emotions of rewarded, free, jolted, and nervous. Hay-like and citrus described an off-balance feeling.

This study present the useful interaction of sensory and emotion data. Using the emotion profiles generated by the 44 emotions on the coffee drinking experience lexicon, we were able to identify sensory drivers for specific emotions elicited by coffee drinking. 


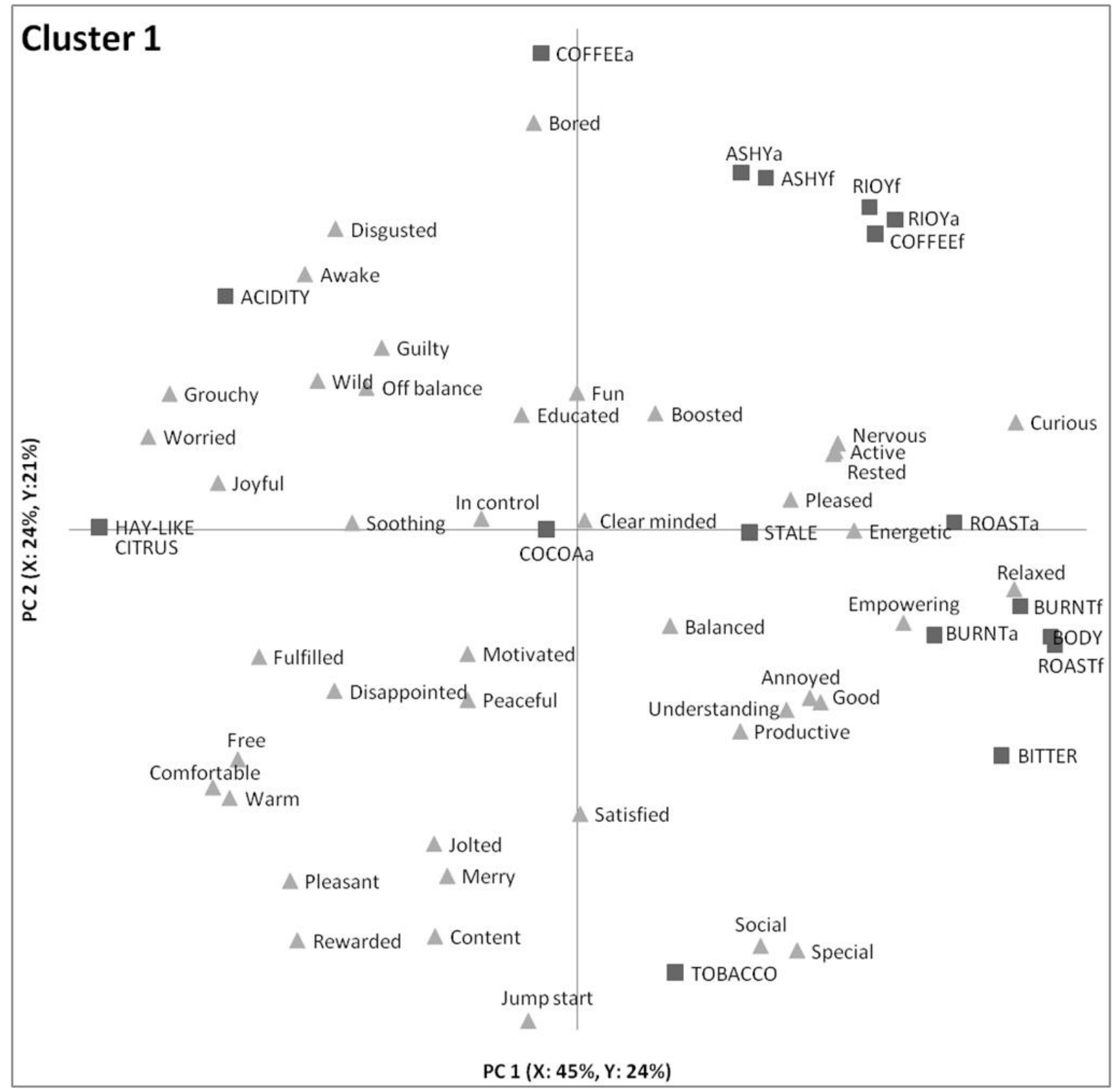

Figure 4.3. Partial Least Square Regression Analysis of Descriptive Sensory and Emotion Data of the Six Coffee Samples for Consumer Cluster 1 


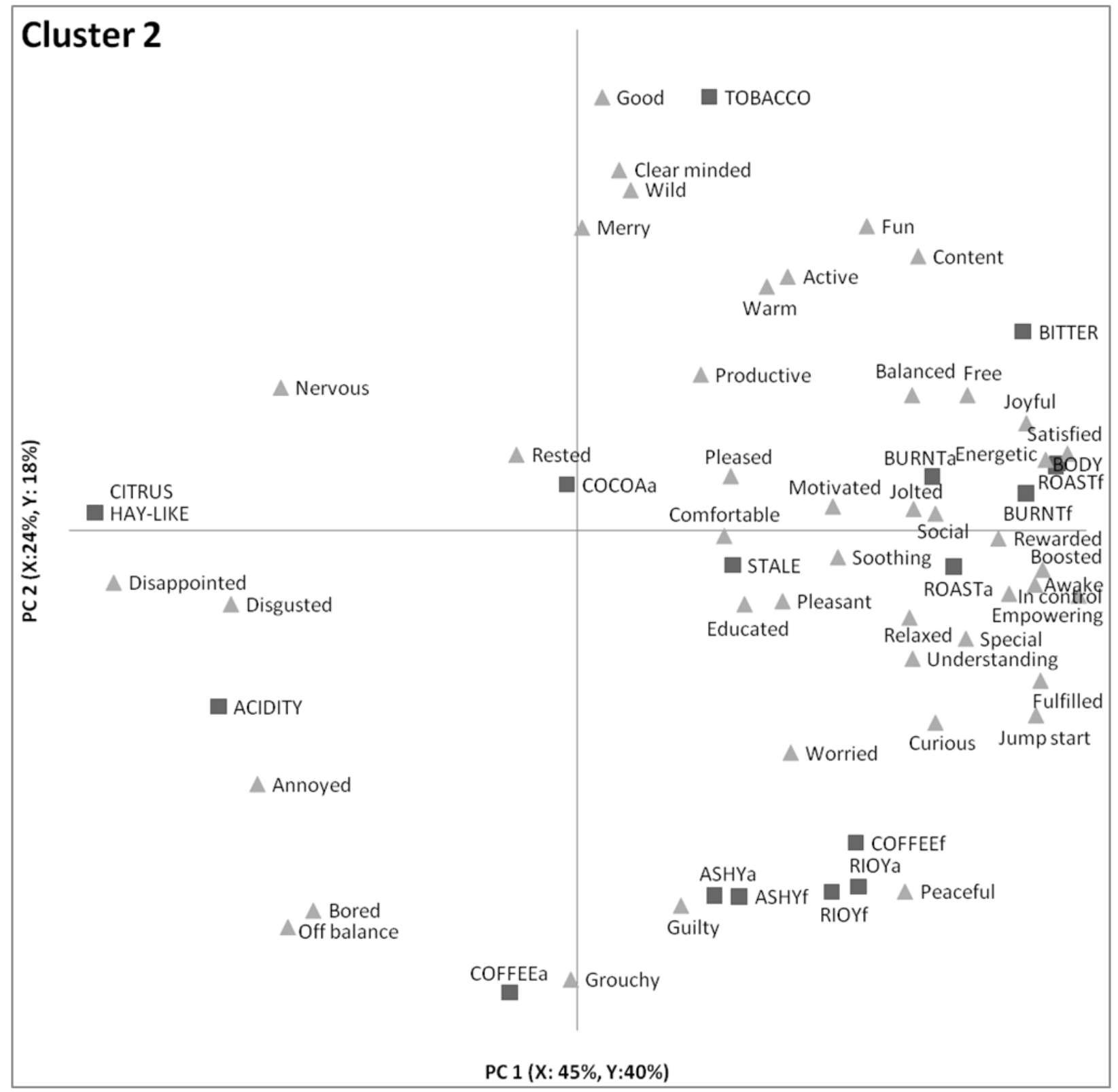

Figure 4.4. Partial Least Square Regression Analysis of Descriptive Sensory and Emotion Data of the Six Coffee Samples for Consumer Cluster 2 


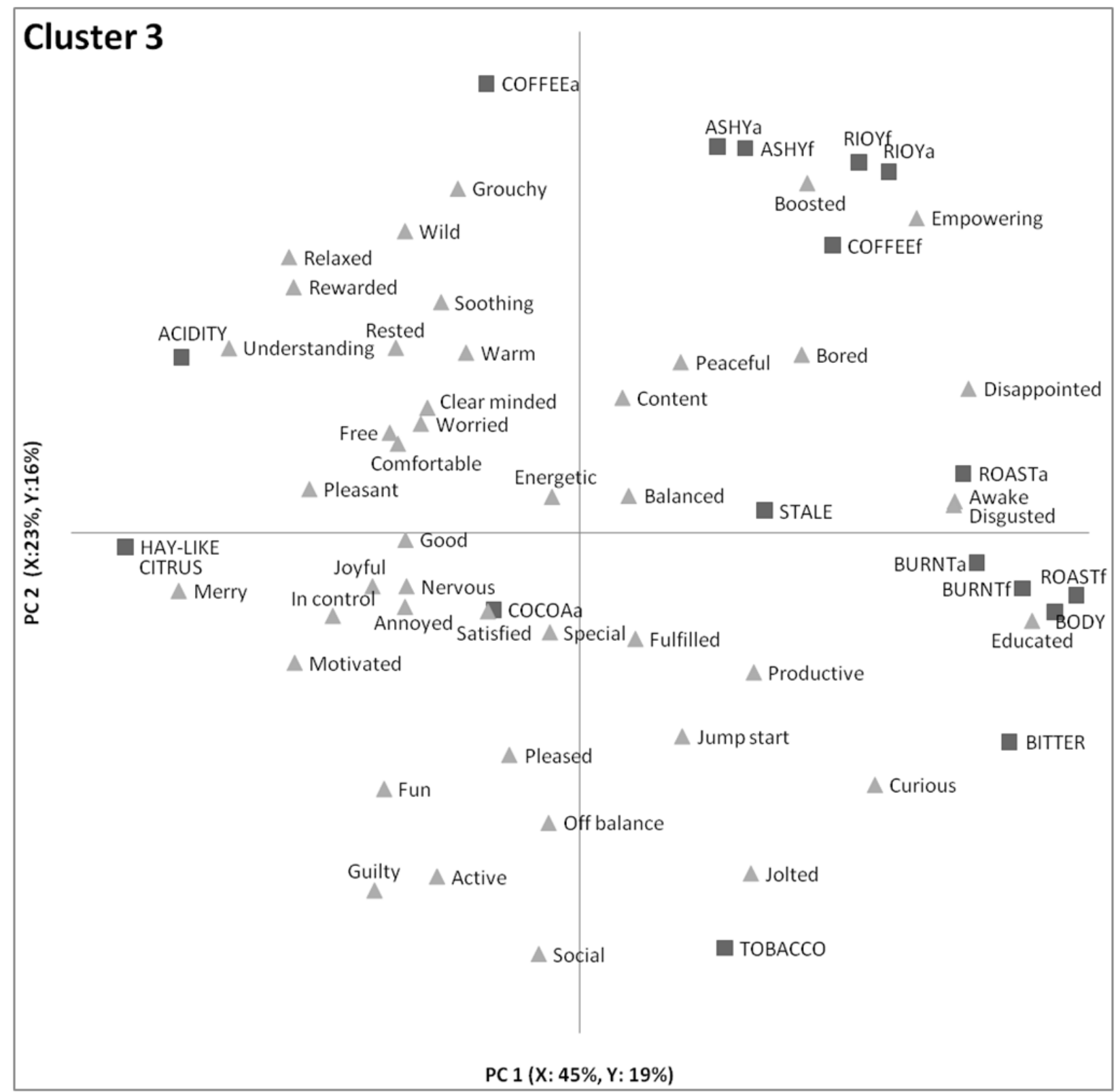

Figure 4.5. Partial Least Square Regression Analysis of Descriptive Sensory and Emotion Data of the Six Coffee Samples for Consumer Cluster 3 


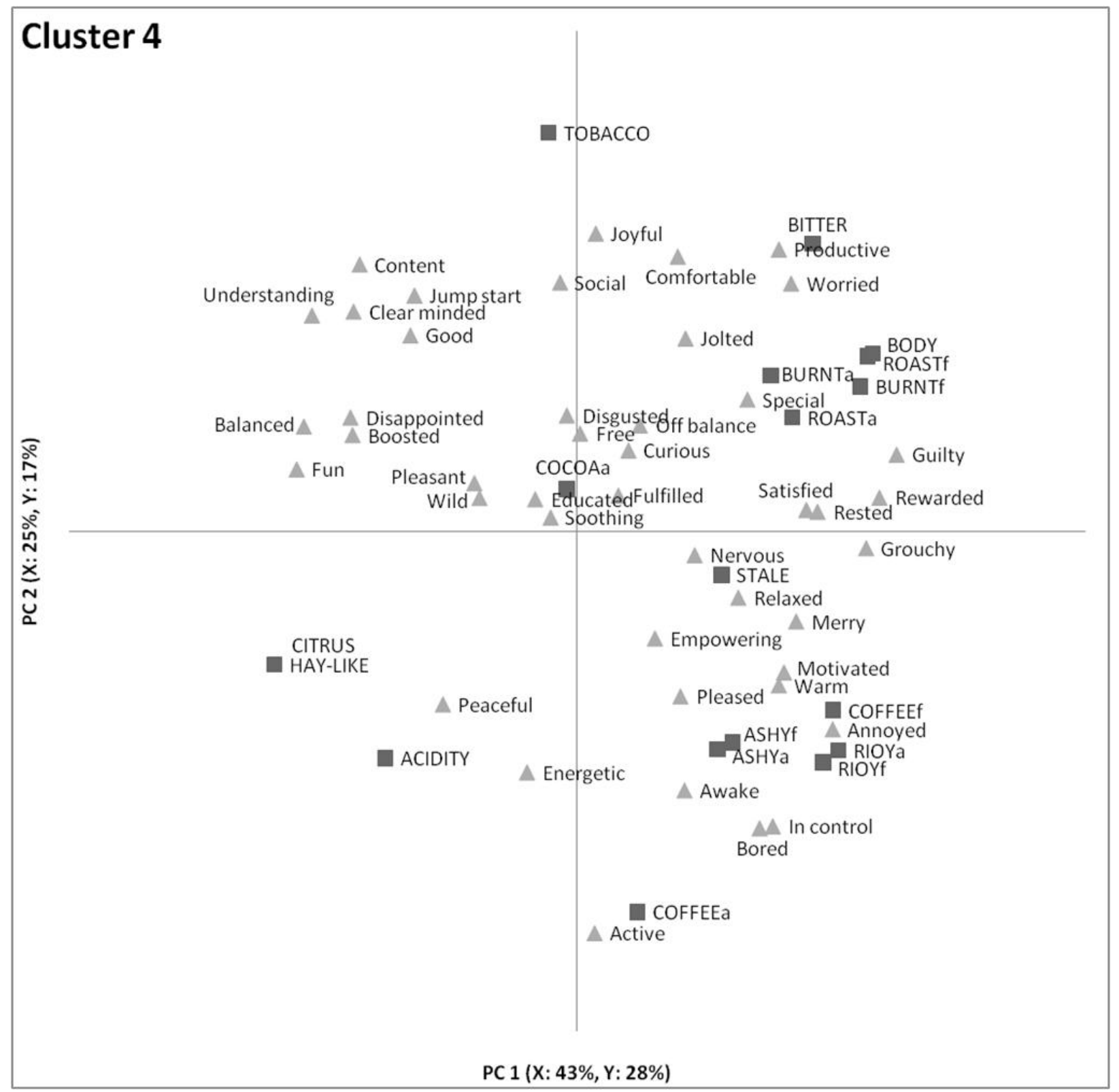

Figure 4.6. Partial Least Square Regression Analysis of Descriptive Sensory and Emotion Data of the Six Coffee Samples for Consumer Cluster 4 


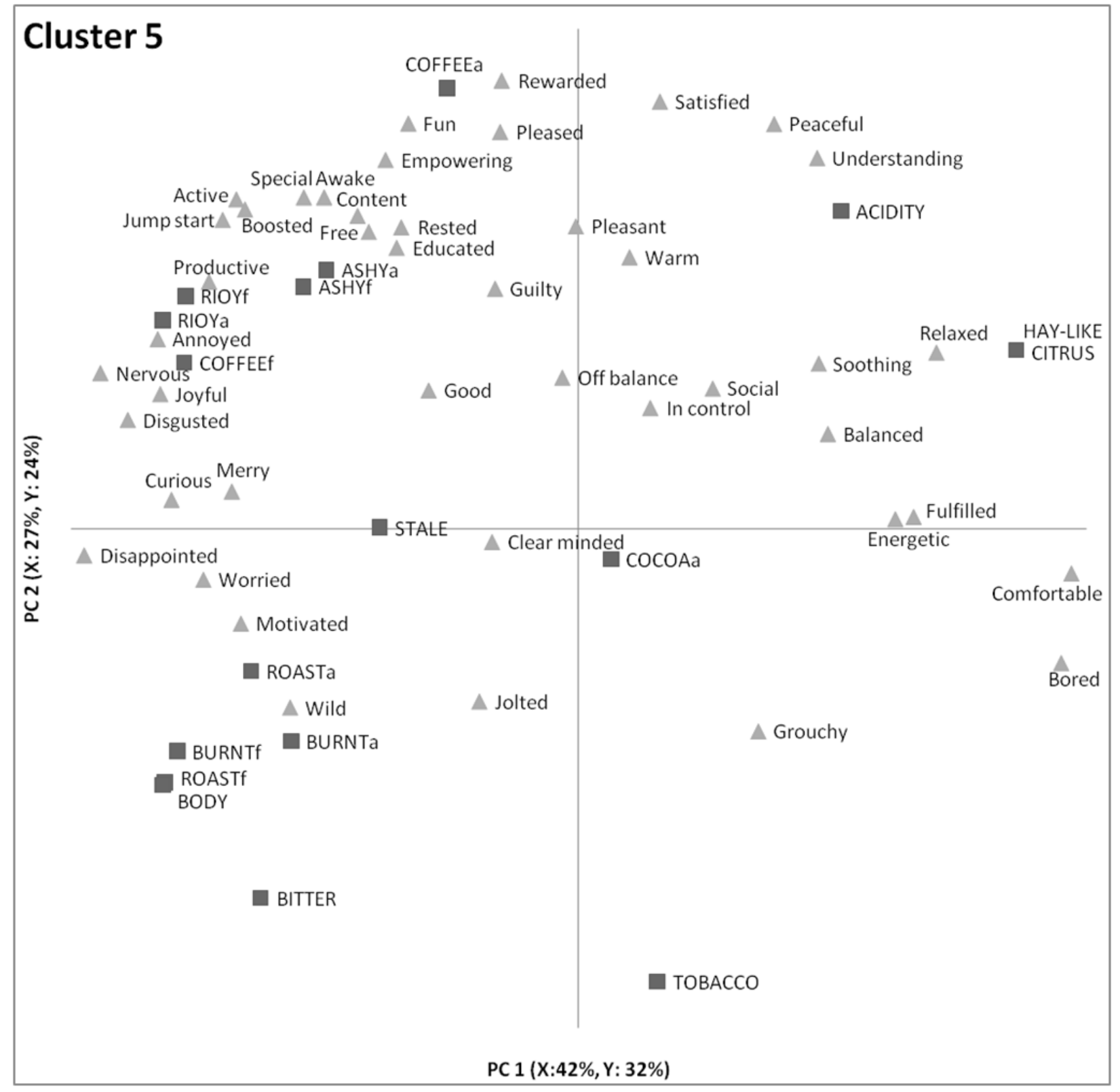

Figure 4.7. Partial Least Square Regression Analysis of Descriptive Sensory and Emotion Data of the Six Coffee Samples for Consumer Cluster 5 


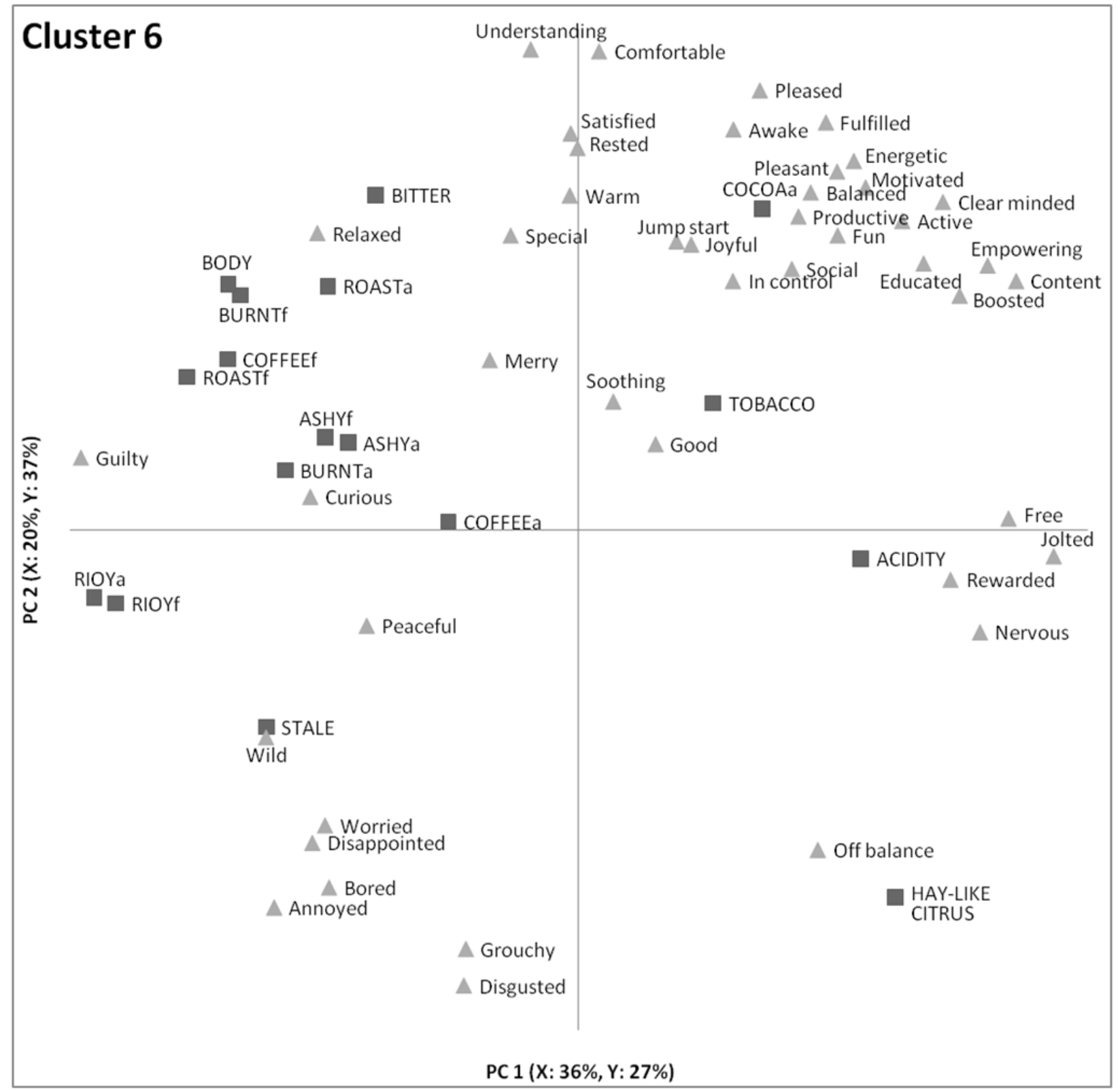

Figure 4.8. Partial Least Square Regression Analysis of Descriptive Sensory and Emotion Data of the Six Coffee Samples for Consumer Cluster 6 


\section{Conclusion}

The PLSR maps indicated that sensory descriptive data can be used to describe emotions profiles elicited by coffee drinking. The maps were used to identify which attributes had an impact on positive or negative emotional responses from various groups of coffee drinkers. In general, coffee aroma, citrus, hay-like, and acidity, elicited negative feelings while cocoa aroma, tobacco, bitter, roast, burnt, and body/mouthfeel generated positive emotions. As consumers have differing likes and dislikes, this study also examined each consumer cluster based on their preferences and identified sensory drivers for the emotions experienced by each cluster.

One interesting finding was that coffee aroma and coffee flavor were observed to be independent of each other in all of the clusters. They even generated opposite emotional responses (positive vs. negative) for coffee users in one cluster. These insights generated by the interaction of sensory and emotion data is valuable to both marketers and product developers by explaining acceptability data and change in consumption or purchase behavior. 


\section{References}

Baggenstoss, J., Poisson, L., Kaegi, R., Perren, R., \& Escher, F. (2008). Coffee roasting and aroma formation: application of different time and temperature conditions. Journal of Agricultural and Food Chemistry, 56, 5836-5846.

Bhumiratana, N., Adhikari, K., \& Chambers, E., IV. Green Coffee Beans to Brewed Coffee: Evolution of Coffee Aroma. LWT Journal of Food Science, Submitted.

Bitnes, J., Ueland, O., Moller, P., \& Martens, M. (2008). Reliability of sensory assessors: Issues of retention and learning. Journal of Sensory Studies, 23, 852-870.

Chambers, D. H., Allison, A., \& Chambers, E., IV. (2004). Training effects on performance of descriptive panelists. Journal of Sensory Studies, 19, 486-499.

Chambers, E., IV, \& Smith, E. A. (1993). Effects of testing experience on performance of trained sensory panelists. Journal of Sensory Studies, 8, 155-166.

Chrea, C., Grandjean, D., Delplanque, S. Cayeux, I., Le Calve, B., Aymard, L., Velazco, M.I., Sander, D., \& Scherer, K. (2009). Mapping the semantic space for the subjective experience of emotional responses to odors. Chemical Senses, 34, 49-62.

Czerny, M., Mayer, F., \&Grosch, W. (1999). Sensory study on the character impact odorants of roasted Arabica coffee. Journal of Agricultural and Food Chemistry, 47, 695-699.

Decazy, F., Avelino, J., Guyot, B., Perriot, J.J., Pineda, C., \& Cilas, C. (2003). Quality of different Honduran coffees in relation to several environments. Journal of Food Science, 68(7), 2356-2361.

Gibson, E.L. (2006). Emotional influences on food choice: Sensory, physiological and psychological pathways. Physiology \& Behavior, 89, 53-61.

Grosch, W. (1998). Flavour of coffee: A review. Nahrung, 42, 344-350.

Illy, A., \& Viani, R. (2005). Espresso coffee: The science of quality. (2 ${ }^{\text {nd }}$ ed.). London: Elsevier Academic Press.

Illy, E. (2002) The complexity of coffee. Scientific American, June 2002.

International Coffee Organization (C Coffee disappearance (consumption) data from 2000 to 2009. Available at http://www.ico.org/historical/2000+/PDF/CONSUMPTION.pdf> Accessed 10.10.

International Coffee Organization - Cup tasting. Available at http://www.ico.org/cup_e.asp. Accessed 10.10. 
King, S.C., \& Meiselman, H.L. (2009) Development of a method to measure consumer emotions associated with foods. Food Quality and Preferences, 21, 168-177.

Macht, M., \& Mueller, J. (2007). Immediate effects of chocolate on experimentally induced mood states. Appetite, 49, 667-674.

Mayer, F., Czerny, M., \& Grosch, W. (1999). Influence of provenance and roast degree on the composition of potent odorants in Arabica coffees. European Food Research \& Technology, 209, 242-250.

Nebesny, E., \& Budryn, G. (2006). Evaluation of sensory attributes of coffee brews from robusta coffee roasted under different conditions. European Food Research \& Technology, 224, 159-165.

Porcherot, C., Delplanque, S., Raviot-Derrien, S., Le Calve, B., Chrea, C., Gaudreau, N., \& Cayeux, I. (2010) How do you feel when you smell this? Optimization of a verbal measurement of odor-elicited emotions. Food Quality and Preference, doi:10.1016/j.foodqual.2010.03.012.

Richins, M.L. (1997). Measuring emotions in the consumption experience. Journal of Consumer Research, 24, 127-146.

Ross, C. F., Pecka, K., \& Weller, K. (2006). Effect of storage conditions on the sensory quality of ground Arabica coffee. Journal of Food Quality, 29, 596-606.

Schenker, S., Heinemann, C., Huber, M., Pompizzi, R., Perren, R., \& Escher, F. (2002). Impact of roasting conditions on the formation of aroma compounds in coffee beans. Journal of Food Science, 67, 1, 60-66.

Sivetz, M. S., \& Desrosier, N. W. (1979). Coffee Technology. Westport, Connecticut: AVI.

Seo, H.S., Hirano, M., Shibato, M., Rakwal, R., Hwang, I.K., \& Masuo, Y. (2008). Effect of coffee bean aroma on the rat brain stressed by sleep deprivation: A selected transcript and 2D get-based proteome analysis. Journal of Agricultural and Food Chemistry, 56, 46654673

Thomson, D.M.H. (2006). Sensory cues for emotional responses to foods \& drinks. International Union of Food Science and Technology (IUFoST). Abstract retrieved from http://dx.doi.org/10.1051/IUFoST:20061091.

Thomson, D.M.H., Crocker, C., \& Marketo, C.G. (2010) Linking sensory characteristics to emotions: An example using dark chocolate. Food Quality and Preference, doi:10.1016/j.foodqual.2010.04.011.

Wolters, C. J. \& Allchurch, E. M. (1994). Effect of training procedure on the performance of descriptive panels. Food Quality and Preference, 5, 203-214. 
Yeretzian, C., Jordan, A., Badoud, R., \& Lindinger, W. (2002). From the green bean to the cup of coffee: Investigating coffee roasting by on-line monitoring of volatiles. European Food Research Technology, 214, 92, 92-104. 


\section{CHAPTER 5 - Conclusions and Future Research}

The developed emotion lexicon for the coffee drinking experience provides researchers with a comprehensive list of emotions that occurs during coffee consumption. The entire lexicon consists of 86 emotion terms, which can be used as a base-line for future research on emotion experienced by coffee drinkers of different cultural and demographic background.

When applying this emotion lexicon to assess emotions expressed by coffee drinkers during the drinking experience, the lexicon was further reduced to 44 terms. These 44 emotions were significant in reflecting the impact of different coffees on emotional experiences of diverse coffee drinkers. Examining each cluster of coffee users, it was clear that each cluster sought differing emotional stimulation from the drinking experience. Some liked coffee that drove highenergy emotions, some liked coffee that elicited low-energy emotions. It is vital for the coffee industry to examine the emotional responses the coffee has on the target coffee users in order to produce a product that match and exceed consumers' expectation. Only because the product receives the high acceptability score does not mean it successfully elicits the right type of emotions for that particular consumer segment.

Over half of the emotions (27 terms) uncovered by this lexicon development were elicited specifically by the coffee drinking experience while 17 emotions were more universal and related to the general product consumption. Additionally, a group of emotions describing a focus mental state (e.g., in-control, motivated, productive, clear-minded) was identified by this research and were not present in previous scales developed for the general food consumption. This suggested that it is necessary to have an emotion lexicon specifically-developed for coffee drinking to uncover the emotional responses consumers sought and experienced during the consumption.

The sensory drivers for the emotional experiences elicited by coffee drinking were determined through the use of a specifically-trained coffee descriptive panel. Partial least squares regression maps of the sensory and emotion demonstrated that the sensory elements of dark roast coffee (roast, burnt, bitter, and body/mouthfeel) drove positive-high energy feelings (e.g., energetic, boosted, productive, jump start) for this population of coffee users. Tobacco and cocoa also elicited positive emotions (e.g., pleasant and content) while citrus, hay-like, acidic, and coffee aroma were associated with negative experiences. 
It is evident that the developed CDE emotion lexicon was successful in providing more information on what is emotionally pleasing to coffee drinkers. It would be beneficial to the coffee industry to use this lexicon to examine the emotions elicited by coffee drinkers of various demographic and cultural profiles and identify the influences of these factors on perceptions and emotional experiences related to coffee drinking. It is also possible to quantitatively map emotion attributes with sensory descriptors and chemical compounds in the coffee products. This will provide a complete connection from the product chemical properties with the sensory characteristics that elicit emotional experiences that drive consumption. The application of this lexicon could also be extended to the emotion study examining the contextual effect of coffee consumption. The coffee manufacturers will ultimately be able define the profiles of the beverage with the emotional experiences each consumer segment seeks for.

This research also serves as a protocol for future emotion lexicon development for other product category in which enjoyment is the main purpose for consumption (e.g., wine or chocolate). Sensory and emotion experiences are the only assessments for the coffee quality, acceptability, and consumption. Therefore, this research did not take into account the impact of the physiological factors (e.g., health, hunger, satiety) that usually accompany most consumption of food and beverages. This protocol could be extended to a product category from which emotional experiences are driven primarily by sensory stimuli and not physiological factors (e.g., health, hunger, satiety). 


\section{Appendix A - Moderator's guide for mini-focus group interview}

\section{Introduction}

Hello, my name is Natnicha. Welcome and thank you for coming.

We have invited you here today to take part in the discussion around coffee drinking and how you feel during the experience.

I want to start by going through the informed consent form with you. (Go through point by point and have them sign and collect them) This is a focus group discussion. It will last approximately one hour. During this time I ask that you are open and honest in sharing your experiences and opinions.

Please let me know if you have negative as well as positive comments. What you say will not affect you or me in anyway. There are no right or wrong answers. I am only interested in hearing your honest opinions. If you find yourself having a totally different set of experiences or a different opinion than the rest of the group, I need to hear it, since you represent a sizable portion of the people out in the real world who just didn't happen to be in the group today to support your view. So I hope that you speak up. If you don't speak up I will be seriously misleading myself, since an important view will not be represented. We are interested in hearing what you are saying, not who is saying it. The report contains only your opinions, not your name. It will not reference you specifically.

I want all of your opinions. Please speak clearly, one at a time. And please share all your side conversation with the rest of the group, not only with your neighbor. We tape record the sessions so be sure to say aloud what you feel.

You are being paid for your time, opinions, and courage to voice your point of view.

\section{Let's start this discussion by introducing yourself by your first name and tell us:}

What is your favorite coffee beverage and what is it that you like about it?

Now please describe the feelings or sensory characters you hope to get from your cup of coffee.

\section{Let's talk about your drinking routine and habit.}


How often do you drink coffee in general?

What time do you normally drink your coffee?

How do you normally drink your coffee?

\section{Describe how you feel when you drink coffee.}

Think about your feelings from the time you start thinking about drinking coffee.

What are the sensations you look for when drinking a cup of coffee?

What is a good or bad cup of coffee?

How do you feel when you drink a good cup of coffee?

How do you feel when you receive a bad cup of coffee?

\section{Now let's talk about your drinking experience...}

\section{At home}

At work
At the café/restaurant

\section{On-the-go}

\begin{tabular}{|l|l|l|l|}
\hline How frequently do you drink coffee? & How frequently do you drink coffee? & $\begin{array}{l}\text { How frequently do you visit a café or } \\
\text { restaurant to drink coffee? }\end{array}$ & $\begin{array}{l}\text { How frequently do you drink coffee on-the- } \\
\text { go? }\end{array}$ \\
\hline $\begin{array}{l}\text { Can you describe your drinking routine? } \\
\text { How do you prepare/drink your coffee? } \\
\text { When do you normally drink your coffee? }\end{array}$ & $\begin{array}{l}\text { Can you describe your drinking routine at } \\
\text { work? How do you prepare/drink your } \\
\text { coffee? When do you normally drink your } \\
\text { coffee? }\end{array}$ & $\begin{array}{l}\text { Can you describe your drinking routine } \\
\text { when you are at the café/restaurant? How } \\
\text { do you normally drink your coffee? When } \\
\text { do you normally drink your coffee at the } \\
\text { café/restaurant? }\end{array}$ & $\begin{array}{l}\text { Can you describe your drinking routine while } \\
\text { on-the-go? How and when do you normally } \\
\text { drink your coffee on-the-go? What are the } \\
\text { occasions that you drink coffee on-the-go? }\end{array}$ \\
\hline & & $\begin{array}{l}\text { Is there a particular café or restaurant that } \\
\text { you will get coffee from? What make you } \\
\text { choose to get coffee there? }\end{array}$ & $\begin{array}{l}\text { Where do you normally get your coffee } \\
\text { from? What make you choose to get coffee } \\
\text { there? }\end{array}$ \\
\hline
\end{tabular}




\begin{tabular}{|c|c|c|c|}
\hline $\begin{array}{l}\text { What make you select a particular coffee? } \\
\text { What are the main things you look for } \\
\text { when selecting coffee to drink at home? }\end{array}$ & $\begin{array}{l}\text { Do you have a variety of coffee to choose } \\
\text { from? What make you choose that } \\
\text { particular coffee? What are the main } \\
\text { things you look for when selecting coffee } \\
\text { to drink at work? }\end{array}$ & $\begin{array}{l}\text { What type of coffee do you order? Can you } \\
\text { explain the decision process? What make } \\
\text { you select a particular coffee? What are } \\
\text { main things you look for when selecting } \\
\text { coffee at the café/restaurant? }\end{array}$ & $\begin{array}{l}\text { What type of coffee do you normally drink } \\
\text { when on-the-go? What make you choose that } \\
\text { particular coffee? What are the main things } \\
\text { you look for when selecting coffee to drink } \\
\text { while on-the-go? }\end{array}$ \\
\hline $\begin{array}{l}\text { What makes you drink coffee at home? } \\
\text { What is the particular reason why you } \\
\text { choose to have coffee at home (or to } \\
\text { make own coffee)? }\end{array}$ & $\begin{array}{l}\text { What makes you drink coffee at work? } \\
\text { What is the particular reason why you } \\
\text { choose to have coffee at work? }\end{array}$ & $\begin{array}{l}\text { What makes you order a coffee at the } \\
\text { café/restaurant? And what makes you stay } \\
\text { and drink your drink there? }\end{array}$ & $\begin{array}{l}\text { What makes you drink coffee while on-the- } \\
\text { go? }\end{array}$ \\
\hline $\begin{array}{l}\text { Can you describe the experience? How do } \\
\text { you feel during the entire experience? } \\
\text { How do you feel when you make your } \\
\text { own coffee? How do you feel when the } \\
\text { coffee is ready? How do you feel while } \\
\text { drinking your coffee at your home? What } \\
\text { do you like about having coffee at home? }\end{array}$ & $\begin{array}{l}\text { Can you describe the experience? How do } \\
\text { you feel when you have coffee at work? } \\
\text { What do you like about having coffee at } \\
\text { work? }\end{array}$ & $\begin{array}{l}\text { Can you describe the experience? How do } \\
\text { you feel during the experience? Imagine } \\
\text { you are at the café for your favorite coffee } \\
\text { drink. How do you feel? How do you feel } \\
\text { when you're sitting down sipping the drink? } \\
\text { What do you like about having coffee at the } \\
\text { café? }\end{array}$ & $\begin{array}{l}\text { Can you describe the experience? How do } \\
\text { you feel when you have coffee while on-the- } \\
\text { go? What do you like having coffee while } \\
\text { on-the-go? }\end{array}$ \\
\hline $\begin{array}{l}\text { Has the experience always been good } \\
\text { (enjoyable, satisfying)? }\end{array}$ & $\begin{array}{l}\text { Has the experience always been good } \\
\text { (enjoyable, satisfying)? }\end{array}$ & $\begin{array}{l}\text { Has the experience always been good } \\
\text { (enjoyable, satisfying)? }\end{array}$ & $\begin{array}{l}\text { Has the experience always been good } \\
\text { (enjoyable, satisfying)? }\end{array}$ \\
\hline $\begin{array}{l}\text { Can you think about a time when you did } \\
\text { not enjoy drinking coffee at home? How } \\
\text { did you feel when the experience was not } \\
\text { good? }\end{array}$ & $\begin{array}{l}\text { Can you think about a time when you did } \\
\text { not enjoy drinking coffee at work? How } \\
\text { did you feel when the experience was not } \\
\text { good? }\end{array}$ & $\begin{array}{l}\text { Can you think about a time when you did } \\
\text { not enjoy drinking coffee at the café? How } \\
\text { did you feel when the experience was not } \\
\text { good? }\end{array}$ & $\begin{array}{l}\text { Can you think about a time when you did not } \\
\text { enjoy drinking coffee on-the-go? How did } \\
\text { you feel when the experience was not good? }\end{array}$ \\
\hline $\begin{array}{l}\text { What is it about the experience that didn't } \\
\text { meet your expectation? How does that } \\
\text { make you feel? }\end{array}$ & $\begin{array}{l}\text { What is it about the experience that didn't } \\
\text { meet your expectation? How does that } \\
\text { make you feel? }\end{array}$ & $\begin{array}{l}\text { What is it about the experience that didn't } \\
\text { meet your expectation? How does that make } \\
\text { you feel? }\end{array}$ & $\begin{array}{l}\text { What is it about the experience that didn't } \\
\text { meet your expectation? How does that make } \\
\text { you feel? }\end{array}$ \\
\hline
\end{tabular}




\begin{tabular}{|l|l|l|l|}
\hline $\begin{array}{l}\text { What was your expectation? How do you } \\
\text { want to feel while having coffee at home? }\end{array}$ & $\begin{array}{l}\text { What was your expectation? How do you } \\
\text { want to feel while having coffee at work? }\end{array}$ & $\begin{array}{l}\text { What was your expectation? How do you } \\
\text { want to feel while having coffee at the } \\
\text { café/restaurant? }\end{array}$ & $\begin{array}{l}\text { What was your expectation? How do you } \\
\text { want to feel while having coffee on-the-go? }\end{array}$ \\
\hline $\begin{array}{l}\text { What is most enjoyable about the coffee } \\
\text { drinking experience at home? }\end{array}$ & $\begin{array}{l}\text { What is most enjoyable about the coffee } \\
\text { drinking experience at work? }\end{array}$ & $\begin{array}{l}\text { What is most enjoyable about the coffee } \\
\text { drinking experience at a café? }\end{array}$ & $\begin{array}{l}\text { What is most enjoyable about the coffee } \\
\text { drinking experience on-the-go? }\end{array}$ \\
\hline $\begin{array}{l}\text { Is there any drawback to coffee drinking? } \\
\text { Is there any drawback to coffee drinking } \\
\text { at home? }\end{array}$ & $\begin{array}{l}\text { Is there any drawback to coffee drinking } \\
\text { at work? }\end{array}$ & $\begin{array}{l}\text { Is there any drawback to drinking coffee at } \\
\text { a café or restaurant? }\end{array}$ & $\begin{array}{l}\text { Is there any drawback to coffee drinking on- } \\
\text { the-go? }\end{array}$ \\
\hline
\end{tabular}

\section{Please sum up the entire coffee drinking experience into 3 to 5 words.}

Give me a set of terms for each good and bad experience. (Discuss about each term, what they mean...) 


\section{Appendix B - Moderator's guide for focus group interview}

\section{Introduction}

Welcome and thank you for coming. We have invited you here today to take part in the discussion about coffee drinking and how you feel during the experience. I want to start by going through the informed consent form with you. (Go though point by point. Have the participant sign and collect the signed form).

This is a focus group discussion. It will last approximately 90 minutes. During this time I ask that you are open and honest in sharing your experiences and thoughts. Please let me know all of your positive and negative experiences and feelings about coffee.

If you find yourself having a totally different set of experiences or opinions than the rest of the group, I need to hear it since each of you represent a sizable portion of your demographic group that did not happen to be here today to support your view. So please speak up, otherwise I will be misleading myself since an important view will not be represented.

I want to hear all of your opinions. Please speak loud and clear, one at a time. We tape record these sessions so be sure to say aloud what you feel. You are being paid for your time, opinions, and courage to voice your point of views.

\section{Encourage participants to take a coffee drink}

Think about your favorite coffee - highlight the terms that represent how you feel.

Think about your bad experience with coffee - highlight the terms that represent how you feel. Think about your regular, every experience with coffee - highlight the terms that represent how you feel.

\section{Write all the terms on the board. Tally. Discuss about each term.}

Look at the list of terms. Discuss definitions, redundancy, suitability, phrases.

Discuss about drinking experience at home, coffee shop, work, on-the-go. Generate more terms and discuss them, if possible. 


\section{Appendix C - Questionnaire for the consumer test}

\section{Good morning!}

Before drinking your coffee, PLEASE INDICATE HOW YOU FEEL using the terms listed below. It is important that you rate each feeling listed below.

\begin{tabular}{|c|c|c|c|c|c|c|c|c|c|c|c|}
\hline Feelings & 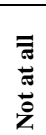 & 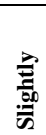 & 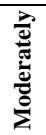 & $B_{D}$ & 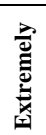 & Feelings & $\begin{array}{l}\overline{\tilde{\sigma}} \\
\text { స } \\
\stackrel{0}{0}\end{array}$ & 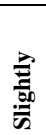 & 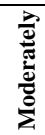 & $\stackrel{\vec{D}}{D}$ & 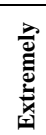 \\
\hline Active & 1 & 2 & 3 & 4 & 5 & In control & 1 & 2 & 3 & 4 & 5 \\
\hline Adventurous & 1 & 2 & 3 & 4 & 5 & Independent & 1 & 2 & 3 & 4 & 5 \\
\hline Affectionate & 1 & 2 & 3 & 4 & 5 & Interested & 1 & 2 & 3 & 4 & 5 \\
\hline Aggressive & 1 & 2 & 3 & 4 & 5 & Intrigued & 1 & 2 & 3 & 4 & 5 \\
\hline Alert & 1 & 2 & 3 & 4 & 5 & Jolted & 1 & 2 & 3 & 4 & 5 \\
\hline Annoyed & 1 & 2 & 3 & 4 & 5 & Joyful & 1 & 2 & 3 & 4 & 5 \\
\hline Attentive & 1 & 2 & 3 & 4 & 5 & Jump start & 1 & 2 & 3 & 4 & 5 \\
\hline Awake & 1 & 2 & 3 & 4 & 5 & Loving & 1 & 2 & 3 & 4 & 5 \\
\hline Balanced & 1 & 2 & 3 & 4 & 5 & Merry & 1 & 2 & 3 & 4 & 5 \\
\hline Boosted & 1 & 2 & 3 & 4 & 5 & Mild & 1 & 2 & 3 & 4 & 5 \\
\hline Bored & 1 & 2 & 3 & 4 & 5 & Motivated & 1 & 2 & 3 & 4 & 5 \\
\hline Calm & 1 & 2 & 3 & 4 & 5 & Nervous & 1 & 2 & 3 & 4 & 5 \\
\hline Clear minded & 1 & 2 & 3 & 4 & 5 & Nostalgic & 1 & 2 & 3 & 4 & 5 \\
\hline Collected & 1 & 2 & 3 & 4 & 5 & Off-balance & 1 & 2 & 3 & 4 & 5 \\
\hline Comfortable & 1 & 2 & 3 & 4 & 5 & Peaceful & 1 & 2 & 3 & 4 & 5 \\
\hline Comforted & 1 & 2 & 3 & 4 & 5 & Pleasant & 1 & 2 & 3 & 4 & 5 \\
\hline Content & 1 & 2 & 3 & 4 & 5 & Pleased & 1 & 2 & 3 & 4 & 5 \\
\hline Cozy & 1 & 2 & 3 & 4 & 5 & Productive & 1 & 2 & 3 & 4 & 5 \\
\hline Cultured & 1 & 2 & 3 & 4 & 5 & Quiet & 1 & 2 & 3 & 4 & 5 \\
\hline Curious & 1 & 2 & 3 & 4 & 5 & Ready & 1 & 2 & 3 & 4 & 5 \\
\hline Daring & 1 & 2 & 3 & 4 & 5 & Relax & 1 & 2 & 3 & 4 & 5 \\
\hline Disappointed & 1 & 2 & 3 & 4 & 5 & Relieved & 1 & 2 & 3 & 4 & 5 \\
\hline Disgusted & 1 & 2 & 3 & 4 & 5 & Rested & 1 & 2 & 3 & 4 & 5 \\
\hline Eager & 1 & 2 & 3 & 4 & 5 & Rewarded & 1 & 2 & 3 & 4 & 5 \\
\hline Educated & 1 & 2 & 3 & 4 & 5 & Sad & 1 & 2 & 3 & 4 & 5 \\
\hline Empowering & 1 & 2 & 3 & 4 & 5 & Safe & 1 & 2 & 3 & 4 & 5 \\
\hline Energetic & 1 & 2 & 3 & 4 & 5 & Satisfied & 1 & 2 & 3 & 4 & 5 \\
\hline Enthusiastic & 1 & 2 & 3 & 4 & 5 & Secure & 1 & 2 & 3 & 4 & 5 \\
\hline Excited & 1 & 2 & 3 & 4 & 5 & Social & 1 & 2 & 3 & 4 & 5 \\
\hline Experimental & 1 & 2 & 3 & 4 & 5 & Soothing & 1 & 2 & 3 & 4 & 5 \\
\hline Focused & 1 & 2 & 3 & 4 & 5 & Special & 1 & 2 & 3 & 4 & 5 \\
\hline Free & 1 & 2 & 3 & 4 & 5 & Stable & 1 & 2 & 3 & 4 & 5 \\
\hline Friendly & 1 & 2 & 3 & 4 & 5 & Steady & 1 & 2 & 3 & 4 & 5 \\
\hline Frustrated & 1 & 2 & 3 & 4 & 5 & Tame & 1 & 2 & 3 & 4 & 5 \\
\hline Fulfilling & 1 & 2 & 3 & 4 & 5 & Tender & 1 & 2 & 3 & 4 & 5 \\
\hline Fun & 1 & 2 & 3 & 4 & 5 & Tired & 1 & 2 & 3 & 4 & 5 \\
\hline Glad & 1 & 2 & 3 & 4 & 5 & Tolerated & 1 & 2 & 3 & 4 & 5 \\
\hline Good & 1 & 2 & 3 & 4 & 5 & Understanding & 1 & 2 & 3 & 4 & 5 \\
\hline Good-natured & 1 & 2 & 3 & 4 & 5 & Upset & 1 & 2 & 3 & 4 & 5 \\
\hline Grouchy & 1 & 2 & 3 & 4 & 5 & Warm & 1 & 2 & 3 & 4 & 5 \\
\hline Guilty & 1 & 2 & 3 & 4 & 5 & Whole & 1 & 2 & 3 & 4 & 5 \\
\hline Happy & 1 & 2 & 3 & 4 & 5 & Wild & 1 & 2 & 3 & 4 & 5 \\
\hline Home & 1 & 2 & 3 & 4 & 5 & Worried & 1 & 2 & 3 & 4 & 5 \\
\hline
\end{tabular}

Please turn the page and wait for your coffee to be served 
Please take few sips of your coffee. Add cream/sugar as necessary.

While you drink your coffee, INDICATE HOW MUCH YOU LIKE THE COFFEE you are drinking.

\begin{tabular}{c|c|c|c|c|c|c|c|c|}
\hline $\begin{array}{c}\text { Dislike } \\
\text { extremely }\end{array}$ & $\begin{array}{c}\text { Dislike } \\
\text { very much }\end{array}$ & $\begin{array}{c}\text { Dislike } \\
\text { moderately }\end{array}$ & $\begin{array}{c}\text { Dislike } \\
\text { slightly }\end{array}$ & $\begin{array}{c}\text { Neither } \\
\text { like } \\
\text { nor dislike }\end{array}$ & $\begin{array}{c}\text { Like } \\
\text { slightly }\end{array}$ & $\begin{array}{c}\text { Like } \\
\text { moderately }\end{array}$ & $\begin{array}{c}\text { Like } \\
\text { very much }\end{array}$ & $\begin{array}{c}\text { Like } \\
\text { extremely }\end{array}$ \\
\hline$\square$ & $\square$ & $\square$ & $\square$ & $\square$ & $\square$ & $\square$ & $\square$ & $\square$ \\
\hline
\end{tabular}

While you drink your coffee, PLEASE INDICATE HOW YOU FEEL using the terms listed below.

It is important that you rate each feeling listed below.

\begin{tabular}{|c|c|c|c|c|c|c|c|c|c|c|c|}
\hline Feelings & 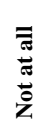 & 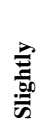 & 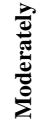 & $\vec{D}$ & 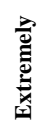 & Feelings & $\begin{array}{l}\overline{\bar{\sigma}} \\
\stackrel{\sigma}{\sigma} \\
\stackrel{0}{z}\end{array}$ & 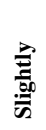 & 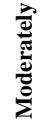 & $\vec{D}_{D}^{D}$ & 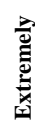 \\
\hline Active & 1 & 2 & 3 & 4 & 5 & In control & 1 & 2 & 3 & 4 & 5 \\
\hline Adventurous & 1 & 2 & 3 & 4 & 5 & Independent & 1 & 2 & 3 & 4 & 5 \\
\hline Affectionate & 1 & 2 & 3 & 4 & 5 & Interested & 1 & 2 & 3 & 4 & 5 \\
\hline Aggressive & 1 & 2 & 3 & 4 & 5 & Intrigued & 1 & 2 & 3 & 4 & 5 \\
\hline Alert & 1 & 2 & 3 & 4 & 5 & Jolted & 1 & 2 & 3 & 4 & 5 \\
\hline Annoyed & 1 & 2 & 3 & 4 & 5 & Joyful & 1 & 2 & 3 & 4 & 5 \\
\hline Attentive & 1 & 2 & 3 & 4 & 5 & Jump start & 1 & 2 & 3 & 4 & 5 \\
\hline Awake & 1 & 2 & 3 & 4 & 5 & Loving & 1 & 2 & 3 & 4 & 5 \\
\hline Balanced & 1 & 2 & 3 & 4 & 5 & Merry & 1 & 2 & 3 & 4 & 5 \\
\hline Boosted & 1 & 2 & 3 & 4 & 5 & Mild & 1 & 2 & 3 & 4 & 5 \\
\hline Bored & 1 & 2 & 3 & 4 & 5 & Motivated & 1 & 2 & 3 & 4 & 5 \\
\hline Calm & 1 & 2 & 3 & 4 & 5 & Nervous & 1 & 2 & 3 & 4 & 5 \\
\hline Clear minded & 1 & 2 & 3 & 4 & 5 & Nostalgic & 1 & 2 & 3 & 4 & 5 \\
\hline Collected & 1 & 2 & 3 & 4 & 5 & Off-balance & 1 & 2 & 3 & 4 & 5 \\
\hline Comfortable & 1 & 2 & 3 & 4 & 5 & Peaceful & 1 & 2 & 3 & 4 & 5 \\
\hline Comforted & 1 & 2 & 3 & 4 & 5 & Pleasant & 1 & 2 & 3 & 4 & 5 \\
\hline Content & 1 & 2 & 3 & 4 & 5 & Pleased & 1 & 2 & 3 & 4 & 5 \\
\hline Cozy & 1 & 2 & 3 & 4 & 5 & Productive & 1 & 2 & 3 & 4 & 5 \\
\hline Cultured & 1 & 2 & 3 & 4 & 5 & Quiet & 1 & 2 & 3 & 4 & 5 \\
\hline Curious & 1 & 2 & 3 & 4 & 5 & Ready & 1 & 2 & 3 & 4 & 5 \\
\hline Daring & 1 & 2 & 3 & 4 & 5 & Relax & 1 & 2 & 3 & 4 & 5 \\
\hline Disappointed & 1 & 2 & 3 & 4 & 5 & Relieved & 1 & 2 & 3 & 4 & 5 \\
\hline Disgusted & 1 & 2 & 3 & 4 & 5 & Rested & 1 & 2 & 3 & 4 & 5 \\
\hline Eager & 1 & 2 & 3 & 4 & 5 & Rewarded & 1 & 2 & 3 & 4 & 5 \\
\hline Educated & 1 & 2 & 3 & 4 & 5 & Sad & 1 & 2 & 3 & 4 & 5 \\
\hline Empowering & 1 & 2 & 3 & 4 & 5 & Safe & 1 & 2 & 3 & 4 & 5 \\
\hline Energetic & 1 & 2 & 3 & 4 & 5 & Satisfied & 1 & 2 & 3 & 4 & 5 \\
\hline Enthusiastic & 1 & 2 & 3 & 4 & 5 & Secure & 1 & 2 & 3 & 4 & 5 \\
\hline Excited & 1 & 2 & 3 & 4 & 5 & Social & 1 & 2 & 3 & 4 & 5 \\
\hline Experimental & 1 & 2 & 3 & 4 & 5 & Soothing & 1 & 2 & 3 & 4 & 5 \\
\hline Focused & 1 & 2 & 3 & 4 & 5 & Special & 1 & 2 & 3 & 4 & 5 \\
\hline Free & 1 & 2 & 3 & 4 & 5 & Stable & 1 & 2 & 3 & 4 & 5 \\
\hline Friendly & 1 & 2 & 3 & 4 & 5 & Steady & 1 & 2 & 3 & 4 & 5 \\
\hline Frustrated & 1 & 2 & 3 & 4 & 5 & Tame & 1 & 2 & 3 & 4 & 5 \\
\hline Fulfilling & 1 & 2 & 3 & 4 & 5 & Tender & 1 & 2 & 3 & 4 & 5 \\
\hline Fun & 1 & 2 & 3 & 4 & 5 & Tired & 1 & 2 & 3 & 4 & 5 \\
\hline Glad & 1 & 2 & 3 & 4 & 5 & Tolerated & 1 & 2 & 3 & 4 & 5 \\
\hline Good & 1 & 2 & 3 & 4 & 5 & Understanding & 1 & 2 & 3 & 4 & 5 \\
\hline Good-natured & 1 & 2 & 3 & 4 & 5 & Upset & 1 & 2 & 3 & 4 & 5 \\
\hline Grouchy & 1 & 2 & 3 & 4 & 5 & Warm & 1 & 2 & 3 & 4 & 5 \\
\hline Guilty & 1 & 2 & 3 & 4 & 5 & Whole & 1 & 2 & 3 & 4 & 5 \\
\hline Hарру & 1 & 2 & 3 & 4 & 5 & Wild & 1 & 2 & 3 & 4 & 5 \\
\hline Home & 1 & 2 & 3 & 4 & 5 & Worried & 1 & 2 & 3 & 4 & 5 \\
\hline
\end{tabular}




\section{Appendix D - Demographic questionnaire}

\section{PLEASE CIRCLE THE APPROPRIATE ANSWER(S)}

\section{Gender:}
(1) Male
(2) Female

2. Age:
(1) 18 to 30
(2) 31 to 45
(3) 46 to 65
(4) Above 65

3. Education background:
(1) Grade School
(4) Some College
(7) Master/Doctoral
(2) High School
(5) Bachelor's Degree
(3) High School Graduate
(6) Associate Degree

4. Annually income:
(1) Less than $\$ 15,000$
(3) $\$ 30,000-49,999$
(2) $\$ 15,000-29,999$
(4) Above $\$ 50,000$

5. Coffee consumption frequency:
(1) 1-2 times/week
(2) 3-5 times/week
(3)Once a day or more

6. Please indicate where you obtain your coffee beverage on a regular basis:
(1) Home
(4) Quick Shop/Vendor
(2) Office
(5) Other (Please specify)
(3) Coffee Shop/Restaurant

7. Do you regularly brew your own coffee?
(1) Yes
(2)No (skip to question 8)

7a. If you brew your coffee, what do you usually buy?
(1) Whole roasted bean
(3) Other (specify)
(2) Ground coffee

7b. If you brew your coffee, where do you buy your coffee from?
(1) Local coffee shop
(3) Grocery store/discount store
(2) Specialty/gourmet store
(4) Other (specify)

8. What type of coffee do you enjoy? (Select all that apply)
(1) Light
(2) Medium
(4) Mild
(3) Dark
(5) Bold
(6) Flavored (Hazelnut, caramel, cinnamon, etc.) 


\section{Appendix E - Emotion terms resulted from Stepwise analyses: all consumers}

\begin{tabular}{|c|l|r|r|}
\hline \multicolumn{5}{|c|}{ All 94 consumers } \\
\cline { 1 - 3 } Step & $\begin{array}{c}\text { Variable } \\
\text { Entered }\end{array}$ & $\begin{array}{c}\text { Partial } \\
\mathbf{R}^{\mathbf{2}}\end{array}$ & $\begin{array}{c}\text { Model } \\
\mathbf{R}^{\mathbf{2}}\end{array}$ \\
\cline { 1 - 1 } $\mathbf{1}$ & Disappointed & 0.109 & 0.109 \\
\hline $\mathbf{2}$ & Content & 0.058 & 0.167 \\
\hline $\mathbf{3}$ & Pleased & 0.0231 & 0.1902 \\
\hline $\mathbf{4}$ & Comfortable & 0.0131 & 0.2033 \\
\hline $\mathbf{5}$ & Social & 0.0093 & 0.2126 \\
\hline $\mathbf{6}$ & Jumpstart & 0.0094 & 0.222 \\
\hline $\mathbf{7}$ & Rested & 0.0088 & 0.2309 \\
\hline $\mathbf{8}$ & Understanding & 0.0078 & 0.2386 \\
\hline $\mathbf{9}$ & In control & 0.007 & 0.2456 \\
\hline $\mathbf{1 0}$ & Curious & 0.006 & 0.2516 \\
\hline $\mathbf{1 1}$ & Jolted & 0.0054 & 0.257 \\
\hline $\mathbf{1 2}$ & Merry & 0.006 & 0.2629 \\
\hline $\mathbf{1 3}$ & Guilty & 0.0057 & 0.2686 \\
\hline $\mathbf{1 4}$ & Satisfied & 0.0049 & 0.2735 \\
\hline $\mathbf{1 5}$ & Annoyed & 0.0046 & 0.2781 \\
\hline $\mathbf{1 6}$ & Wild & 0.0037 & 0.2818 \\
\hline $\mathbf{1 7}$ & Peaceful & 0.0038 & 0.2856 \\
\hline $\mathbf{1 8}$ & Soothing & 0.004 & 0.2896 \\
\hline $\mathbf{1 9}$ & Off balance & 0.0033 & 0.2929 \\
\hline $\mathbf{2 0}$ & Balanced & 0.0031 & 0.296 \\
\hline $\mathbf{2 1}$ & Empowering & 0.0035 & 0.2996 \\
\hline $\mathbf{2 2}$ & Boosted & 0.0029 & 0.3024 \\
\hline $\mathbf{2 3}$ & Disgusted & 0.0028 & 0.3053 \\
\hline $\mathbf{2 4}$ & Grouchy & 0.0029 & 0.3082 \\
\hline $\mathbf{2 5}$ & Warm & 0.0028 & 0.311 \\
\hline
\end{tabular}




\section{Appendix F - Emotion Terms resulted from Stepwise analyses: by consumer clusters}

\begin{tabular}{|r|l|r|r|}
\hline \multicolumn{4}{|c|}{ Cluster 1 } \\
\hline Step & $\begin{array}{c}\text { Variable } \\
\text { Entered }\end{array}$ & $\begin{array}{c}\text { Partial } \\
\mathbf{R}^{\mathbf{2}}\end{array}$ & $\begin{array}{c}\text { Model } \\
\mathbf{R}^{\mathbf{2}}\end{array}$ \\
\hline $\mathbf{1}$ & Disappointed & 0.1031 & 0.1031 \\
\hline $\mathbf{2}$ & Annoyed & 0.0547 & 0.1578 \\
\hline $\mathbf{3}$ & Jolted & 0.0449 & 0.2027 \\
\hline $\mathbf{4}$ & Content & 0.0427 & 0.2455 \\
\hline $\mathbf{5}$ & Comfortable & 0.056 & 0.3015 \\
\hline $\mathbf{6}$ & Motivated & 0.0333 & 0.3347 \\
\hline $\mathbf{7}$ & In control & 0.0428 & 0.3776 \\
\hline $\mathbf{8}$ & Awake & 0.0267 & 0.4042 \\
\hline $\mathbf{9}$ & Worried & 0.041 & 0.4452 \\
\hline $\mathbf{1 0}$ & Affectionate & 0.0314 & 0.4766 \\
\hline $\mathbf{1 1}$ & Warm & 0.0268 & 0.5034 \\
\hline $\mathbf{1 2}$ & Secure & 0.0222 & 0.5256 \\
\hline $\mathbf{1 3}$ & Intrigued & 0.0205 & 0.5461 \\
\hline $\mathbf{1 4}$ & Special & 0.0235 & 0.5696 \\
\hline $\mathbf{1 5}$ & Interested & 0.0231 & 0.5926 \\
\hline $\mathbf{1 6}$ & Loving & 0.0153 & 0.608 \\
\hline $\mathbf{1 7}$ & Cozy & 0.0176 & 0.6255 \\
\hline $\mathbf{1 8}$ & Tolerated & 0.0147 & 0.6403 \\
\hline $\mathbf{1 9}$ & Fulfilling & 0.0166 & 0.6569 \\
\hline $\mathbf{2 0}$ & Friendly & 0.0098 & 0.6667 \\
\hline $\mathbf{2 1}$ & Nervous & 0.0112 & 0.6779 \\
\hline $\mathbf{2 2}$ & Bored & 0.0123 & 0.6902 \\
\hline $\mathbf{2 3}$ & Active & 0.0118 & 0.702 \\
\hline $\mathbf{2 4}$ & Productive & 0.0102 & 0.7121 \\
\hline $\mathbf{2 5}$ & Good & 0.0127 & 0.7248 \\
\hline $\mathbf{2 6}$ & Collected & 0.0124 & 0.7373 \\
\hline $\mathbf{2 7}$ & Frustrated & 0.0069 & 0.7441 \\
\hline $\mathbf{2 8}$ & Safe & 0.007 & 0.7511 \\
\hline $\mathbf{2 9}$ & Energetic & 0.0096 & 0.7608 \\
\hline $\mathbf{3 0}$ & Free & 0.0065 & 0.7673 \\
\hline
\end{tabular}

\begin{tabular}{|r|l|r|r|}
\hline \multicolumn{4}{|c|}{ Cluster 2 } \\
\hline Step & $\begin{array}{c}\text { Variable } \\
\text { Entered }\end{array}$ & $\begin{array}{c}\text { Partial } \\
\mathbf{R}^{\mathbf{2}}\end{array}$ & $\begin{array}{c}\text { Model } \\
\mathbf{R}^{\mathbf{2}}\end{array}$ \\
\hline $\mathbf{1}$ & Alert & 0.1288 & 0.1288 \\
\hline $\mathbf{2}$ & Grouchy & 0.108 & 0.2368 \\
\hline $\mathbf{3}$ & Collected & 0.076 & 0.3127 \\
\hline $\mathbf{4}$ & Guilty & 0.0642 & 0.377 \\
\hline $\mathbf{5}$ & Disappointed & 0.0594 & 0.4364 \\
\hline $\mathbf{6}$ & Tired & 0.0318 & 0.4682 \\
\hline $\mathbf{7}$ & Special & 0.0331 & 0.5013 \\
\hline $\mathbf{8}$ & Empowering & 0.0303 & 0.5317 \\
\hline $\mathbf{9}$ & Energetic & 0.0409 & 0.5726 \\
\hline $\mathbf{1 0}$ & Worried & 0.0258 & 0.5984 \\
\hline $\mathbf{1 1}$ & Bored & 0.0265 & 0.6249 \\
\hline $\mathbf{1 2}$ & Satisfied & 0.0179 & 0.6428 \\
\hline $\mathbf{1 3}$ & Annoyed & 0.0224 & 0.6652 \\
\hline $\mathbf{1 4}$ & Off balance & 0.0136 & 0.6788 \\
\hline $\mathbf{1 5}$ & Joyful & 0.0178 & 0.6966 \\
\hline $\mathbf{1 6}$ & Tame & 0.0136 & 0.7103 \\
\hline $\mathbf{1 7}$ & Focused & 0.012 & 0.7223 \\
\hline $\mathbf{1 8}$ & Social & 0.0132 & 0.7355 \\
\hline $\mathbf{1 9}$ & Adventurous & 0.0159 & 0.7514 \\
\hline $\mathbf{2 0}$ & Pleasant & 0.013 & 0.7645 \\
\hline $\mathbf{2 1}$ & Home & 0.0144 & 0.7789 \\
\hline $\mathbf{2 2}$ & Loving & 0.0089 & 0.7878 \\
\hline $\mathbf{2 3}$ & Clear minded & 0.0106 & 0.7983 \\
\hline $\mathbf{2 4}$ & Nervous & 0.0081 & 0.8065 \\
\hline $\mathbf{2 5}$ & Jumpstart & 0.0081 & 0.8146 \\
\hline & & & \\
\hline
\end{tabular}




\begin{tabular}{|r|l|c|c|}
\hline \multicolumn{4}{|c|}{ Cluster 3 } \\
\hline Step & $\begin{array}{c}\text { Variable } \\
\text { Entered }\end{array}$ & $\begin{array}{c}\text { Partial } \\
\mathbf{R}^{\mathbf{2}}\end{array}$ & $\begin{array}{c}\text { Model } \\
\mathbf{R}^{\mathbf{2}}\end{array}$ \\
\hline $\mathbf{1}$ & Pleased & 0.1439 & 0.1439 \\
\hline $\mathbf{2}$ & Balanced & 0.0814 & 0.2253 \\
\hline $\mathbf{3}$ & Safe & 0.0499 & 0.2751 \\
\hline $\mathbf{4}$ & Tender & 0.0481 & 0.3232 \\
\hline $\mathbf{5}$ & Tame & 0.0237 & 0.3469 \\
\hline $\mathbf{6}$ & Nervous & 0.0163 & 0.3632 \\
\hline $\mathbf{7}$ & Curious & 0.0205 & 0.3838 \\
\hline $\mathbf{8}$ & Rested & 0.0176 & 0.4014 \\
\hline $\mathbf{9}$ & Excited & 0.0149 & 0.4163 \\
\hline $\mathbf{1 0}$ & Experimental & 0.0263 & 0.4426 \\
\hline $\mathbf{1 1}$ & Secure & 0.0122 & 0.4548 \\
\hline $\mathbf{1 2}$ & Attentive & 0.0121 & 0.4669 \\
\hline $\mathbf{1 3}$ & Upset & 0.0123 & 0.4792 \\
\hline
\end{tabular}

\begin{tabular}{|r|l|r|r|}
\hline \multicolumn{4}{|c|}{ Cluster 4 } \\
\hline Step & $\begin{array}{c}\text { Variable } \\
\text { Entered }\end{array}$ & $\begin{array}{c}\text { Partial } \\
\mathbf{R}^{\mathbf{2}}\end{array}$ & $\begin{array}{c}\text { Model } \\
\mathbf{R}^{\mathbf{2}}\end{array}$ \\
\hline $\mathbf{1}$ & Nervous & 0.0947 & 0.0947 \\
\hline $\mathbf{2}$ & Worried & 0.0888 & 0.1835 \\
\hline $\mathbf{3}$ & Independent & 0.0898 & 0.2733 \\
\hline $\mathbf{4}$ & Satisfied & 0.0605 & 0.3338 \\
\hline $\mathbf{5}$ & Good natured & 0.069 & 0.4028 \\
\hline $\mathbf{6}$ & Jumpstart & 0.0459 & 0.4487 \\
\hline $\mathbf{7}$ & Guilty & 0.0339 & 0.4825 \\
\hline $\mathbf{8}$ & Ready & 0.0391 & 0.5216 \\
\hline $\mathbf{9}$ & Affectionate & 0.0354 & 0.557 \\
\hline $\mathbf{1 0}$ & Glad & 0.0361 & 0.5932 \\
\hline $\mathbf{1 1}$ & Calm & 0.0268 & 0.6199 \\
\hline $\mathbf{1 2}$ & Clear minded & 0.0302 & 0.6502 \\
\hline $\mathbf{1 3}$ & In control & 0.0221 & 0.6723 \\
\hline $\mathbf{1 4}$ & Free & 0.0221 & 0.6944 \\
\hline $\mathbf{1 5}$ & Relax & 0.0189 & 0.7133 \\
\hline $\mathbf{1 6}$ & Awake & 0.0188 & 0.7321 \\
\hline $\mathbf{1 7}$ & Educated & 0.0271 & 0.7592 \\
\hline $\mathbf{1 8}$ & Fulfilling & 0.0193 & 0.7785 \\
\hline $\mathbf{1 9}$ & Balanced & 0.0112 & 0.7897 \\
\hline $\mathbf{2 0}$ & Empowering & 0.016 & 0.8057 \\
\hline $\mathbf{2 1}$ & Special & 0.0222 & 0.8279 \\
\hline $\mathbf{2 2}$ & Relieved & 0.0141 & 0.8421 \\
\hline $\mathbf{2 3}$ & Off balance & 0.011 & 0.853 \\
\hline $\mathbf{2 4}$ & Steady & 0.0092 & 0.8623 \\
\hline $\mathbf{2 5}$ & Stable & 0.0256 & 0.8878 \\
\hline $\mathbf{2 6}$ & Attentive & 0.0128 & 0.9007 \\
\hline & & & \\
\hline
\end{tabular}




\begin{tabular}{|r|l|r|r|}
\hline \multicolumn{4}{|c|}{ Cluster 5 } \\
\hline Step & $\begin{array}{c}\text { Variable } \\
\text { Entered }\end{array}$ & $\begin{array}{c}\text { Partial } \\
\mathbf{R}^{\mathbf{2}}\end{array}$ & $\begin{array}{c}\text { Model } \\
\mathbf{R}^{\mathbf{2}}\end{array}$ \\
\hline $\mathbf{1}$ & Free & 0.24 & 0.24 \\
\hline $\mathbf{2}$ & Focused & 0.0961 & 0.3361 \\
\hline $\mathbf{3}$ & Bored & 0.0808 & 0.4169 \\
\hline $\mathbf{4}$ & Productive & 0.0723 & 0.4892 \\
\hline $\mathbf{5}$ & Fulfilling & 0.0816 & 0.5708 \\
\hline $\mathbf{6}$ & Pleased & 0.049 & 0.6198 \\
\hline $\mathbf{7}$ & Active & 0.0485 & 0.6683 \\
\hline $\mathbf{8}$ & Comforted & 0.0448 & 0.7131 \\
\hline $\mathbf{9}$ & Aggressive & 0.0417 & 0.7548 \\
\hline $\mathbf{1 0}$ & Grouchy & 0.0551 & 0.8098 \\
\hline $\mathbf{1 1}$ & Disgusted & 0.0241 & 0.834 \\
\hline $\mathbf{1 2}$ & Good & 0.0246 & 0.8585 \\
\hline $\mathbf{1 3}$ & Cultured & 0.0286 & 0.8872 \\
\hline $\mathbf{1 4}$ & Awake & 0.0183 & 0.9054 \\
\hline $\mathbf{1 5}$ & Tired & 0.0188 & 0.9242 \\
\hline $\mathbf{1 6}$ & Nervous & 0.013 & 0.9372 \\
\hline $\mathbf{1 7}$ & Upset & 0.013 & 0.9502 \\
\hline $\mathbf{1 8}$ & Annoyed & 0.0107 & 0.9609 \\
\hline $\mathbf{1 9}$ & Merry & 0.0163 & 0.9772 \\
\hline
\end{tabular}

\begin{tabular}{|r|l|r|r|}
\hline \multicolumn{4}{|c|}{ Cluster 6 } \\
\hline Step & $\begin{array}{c}\text { Variable } \\
\text { Entered }\end{array}$ & $\begin{array}{c}\text { Partial } \\
\mathbf{R}^{\mathbf{2}}\end{array}$ & $\begin{array}{c}\text { Model } \\
\mathbf{R}^{\mathbf{2}}\end{array}$ \\
\hline $\mathbf{1}$ & Content & 0.3192 & 0.3192 \\
\hline $\mathbf{2}$ & Frustrated & 0.1051 & 0.4242 \\
\hline $\mathbf{3}$ & Peaceful & 0.068 & 0.4922 \\
\hline $\mathbf{4}$ & Satisfied & 0.0538 & 0.546 \\
\hline $\mathbf{5}$ & Wild & 0.0457 & 0.5917 \\
\hline $\mathbf{6}$ & Merry & 0.036 & 0.6277 \\
\hline $\mathbf{7}$ & Pleasant & 0.0334 & 0.6611 \\
\hline $\mathbf{8}$ & Social & 0.0298 & 0.6909 \\
\hline $\mathbf{9}$ & Boosted & 0.0304 & 0.7213 \\
\hline $\mathbf{1 0}$ & Daring & 0.0199 & 0.7412 \\
\hline $\mathbf{1 1}$ & Relieved & 0.0231 & 0.7643 \\
\hline $\mathbf{1 2}$ & Annoyed & 0.0276 & 0.7919 \\
\hline $\mathbf{1 3}$ & Comfortable & 0.0216 & 0.8135 \\
\hline $\mathbf{1 4}$ & Friendly & 0.0254 & 0.8388 \\
\hline $\mathbf{1 5}$ & Independent & 0.0226 & 0.8615 \\
\hline $\mathbf{1 6}$ & Interested & 0.0191 & 0.8806 \\
\hline $\mathbf{1 7}$ & Clear minded & 0.0191 & 0.8997 \\
\hline $\mathbf{1 8}$ & Glad & 0.0095 & 0.9092 \\
\hline $\mathbf{1 9}$ & Energetic & 0.0148 & 0.9239 \\
\hline $\mathbf{2 0}$ & Fulfilling & 0.0159 & 0.9399 \\
\hline & & & \\
\hline
\end{tabular}




\section{Appendix G - Emotion terms resulted from Stepwise Analyses: by product clusters}

\begin{tabular}{|r|l|r|r|}
\hline \multicolumn{4}{|c|}{ BREAKFAST } \\
\hline Step & $\begin{array}{c}\text { Variable } \\
\text { Entered }\end{array}$ & $\begin{array}{c}\text { Partial } \\
\mathbf{R}^{\mathbf{2}}\end{array}$ & $\begin{array}{c}\text { Model } \\
\mathbf{R}^{\mathbf{2}}\end{array}$ \\
\hline $\mathbf{1}$ & Rewarded & 0.2109 & 0.2109 \\
\hline $\mathbf{2}$ & Disappointed & 0.1159 & 0.3268 \\
\hline $\mathbf{3}$ & Aggressive & 0.0524 & 0.3792 \\
\hline $\mathbf{4}$ & Glad & 0.056 & 0.4352 \\
\hline $\mathbf{5}$ & Quiet & 0.0488 & 0.484 \\
\hline $\mathbf{6}$ & Pleasant & 0.0387 & 0.5227 \\
\hline $\mathbf{7}$ & Free & 0.0295 & 0.5522 \\
\hline $\mathbf{8}$ & Rested & 0.0332 & 0.5854 \\
\hline $\mathbf{9}$ & Special & 0.0209 & 0.6064 \\
\hline $\mathbf{1 0}$ & Social & 0.0263 & 0.6327 \\
\hline $\mathbf{1 1}$ & Active & 0.0219 & 0.6546 \\
\hline $\mathbf{1 2}$ & Intrigued & 0.0152 & 0.6697 \\
\hline $\mathbf{1 3}$ & Relax & 0.0177 & 0.6875 \\
\hline $\mathbf{1 4}$ & Mild & 0.0195 & 0.7069 \\
\hline $\mathbf{1 5}$ & Grouchy & 0.0179 & 0.7249 \\
\hline $\mathbf{1 6}$ & Peaceful & 0.0207 & 0.7456 \\
\hline $\mathbf{1 7}$ & Awake & 0.0243 & 0.7698 \\
\hline $\mathbf{1 8}$ & Disgusted & 0.0148 & 0.7846 \\
\hline $\mathbf{1 9}$ & Worried & 0.0146 & 0.7992 \\
\hline $\mathbf{2 0}$ & Fulfilling & 0.0107 & 0.8099 \\
\hline $\mathbf{2 1}$ & Satisfied & 0.0075 & 0.8174 \\
\hline & & & \\
\hline
\end{tabular}

\begin{tabular}{|r|l|r|r|}
\hline \multicolumn{4}{|c|}{ SUMATRA } \\
\hline Step & $\begin{array}{c}\text { Variable } \\
\text { Entered }\end{array}$ & $\begin{array}{c}\text { Partial } \\
\mathbf{R}^{\mathbf{2}}\end{array}$ & $\begin{array}{c}\text { Model } \\
\mathbf{R}^{\mathbf{2}}\end{array}$ \\
\hline $\mathbf{1}$ & Satisfied & 0.1593 & 0.1593 \\
\hline $\mathbf{2}$ & Secure & 0.1234 & 0.2827 \\
\hline $\mathbf{3}$ & Cultured & 0.0528 & 0.3355 \\
\hline $\mathbf{4}$ & Annoyed & 0.0411 & 0.3766 \\
\hline $\mathbf{5}$ & Off balance & 0.0284 & 0.405 \\
\hline $\mathbf{6}$ & Balanced & 0.0313 & 0.4363 \\
\hline $\mathbf{7}$ & Relieved & 0.0191 & 0.4554 \\
\hline $\mathbf{8}$ & Relax & 0.0201 & 0.4755 \\
\hline $\mathbf{9}$ & Curious & 0.024 & 0.4995 \\
\hline $\mathbf{1 0}$ & Upset & 0.0241 & 0.5236 \\
\hline $\mathbf{1 1}$ & Jumpstart & 0.0205 & 0.5441 \\
\hline $\mathbf{1 2}$ & Productive & 0.0154 & 0.5595 \\
\hline $\mathbf{1 3}$ & Rewarded & 0.0222 & 0.5816 \\
\hline $\mathbf{1 4}$ & Special & 0.0229 & 0.6045 \\
\hline $\mathbf{1 5}$ & Tolerated & 0.0195 & 0.624 \\
\hline $\mathbf{1 6}$ & Merry & 0.0242 & 0.6482 \\
\hline $\mathbf{1 7}$ & Bored & 0.0155 & 0.6636 \\
\hline
\end{tabular}




\begin{tabular}{|r|l|r|r|}
\hline \multicolumn{4}{|c|}{ ITALIAN } \\
\hline Step & $\begin{array}{c}\text { Variable } \\
\text { Entered }\end{array}$ & $\begin{array}{c}\text { Partial } \\
\mathbf{R}^{\mathbf{2}}\end{array}$ & $\begin{array}{c}\text { Model } \\
\mathbf{R}^{\mathbf{2}}\end{array}$ \\
\hline $\mathbf{1}$ & Balanced & 0.1582 & 0.1582 \\
\hline $\mathbf{2}$ & Educated & 0.0623 & 0.2205 \\
\hline $\mathbf{3}$ & Ready & 0.0361 & 0.2566 \\
\hline $\mathbf{4}$ & Pleased & 0.0485 & 0.3051 \\
\hline $\mathbf{5}$ & Tired & 0.0482 & 0.3533 \\
\hline $\mathbf{6}$ & Comfortable & 0.0285 & 0.3818 \\
\hline $\mathbf{7}$ & Bored & 0.0285 & 0.4104 \\
\hline $\mathbf{8}$ & Nervous & 0.0341 & 0.4445 \\
\hline $\mathbf{9}$ & Safe & 0.0269 & 0.4714 \\
\hline $\mathbf{1 0}$ & Loving & 0.0189 & 0.4904 \\
\hline $\mathbf{1 1}$ & Joyful & 0.0246 & 0.515 \\
\hline $\mathbf{1 2}$ & Fulfilling & 0.0174 & 0.5323 \\
\hline $\mathbf{1 3}$ & Energetic & 0.0183 & 0.5507 \\
\hline $\mathbf{1 4}$ & Relax & 0.0131 & 0.5638 \\
\hline $\mathbf{1 5}$ & Awake & 0.0126 & 0.5763 \\
\hline $\mathbf{1 6}$ & Good natured & 0.0158 & 0.5921 \\
\hline $\mathbf{1 7}$ & Special & 0.0185 & 0.6106 \\
\hline $\mathbf{1 8}$ & Warm & 0.0139 & 0.6245 \\
\hline $\mathbf{1 9}$ & Calm & 0.0213 & 0.6457 \\
\hline $\mathbf{2 0}$ & Annoyed & 0.03 & 0.6757 \\
\hline $\mathbf{2 1}$ & Home & 0.0164 & 0.6921 \\
\hline $\mathbf{2 2}$ & Off balance & 0.0122 & 0.7043 \\
\hline $\mathbf{2 3}$ & Free & 0.01 & 0.7143 \\
\hline $\mathbf{2 4}$ & Friendly & 0.0103 & 0.7246 \\
\hline $\mathbf{2 5}$ & Attentive & 0.0107 & 0.7352 \\
\hline $\mathbf{2 6}$ & Sad & 0.0091 & 0.7443 \\
\hline $\mathbf{2 7}$ & Worried & 0.0094 & 0.7537 \\
\hline & & & \\
\hline
\end{tabular}

\begin{tabular}{|r|l|r|r|}
\hline \multicolumn{4}{|c|}{ NANTUCKET } \\
\hline Step & $\begin{array}{c}\text { Variable } \\
\text { Entered }\end{array}$ & $\begin{array}{c}\text { Partial } \\
\mathbf{R}^{\mathbf{2}}\end{array}$ & $\begin{array}{c}\text { Model } \\
\mathbf{R}^{\mathbf{2}}\end{array}$ \\
\hline $\mathbf{1}$ & Upset & 0.1833 & 0.1833 \\
\hline $\mathbf{2}$ & Rewarded & 0.0458 & 0.229 \\
\hline $\mathbf{3}$ & Rested & 0.0915 & 0.3206 \\
\hline $\mathbf{4}$ & Social & 0.0385 & 0.3591 \\
\hline $\mathbf{5}$ & Disgusted & 0.0513 & 0.4104 \\
\hline $\mathbf{6}$ & Nervous & 0.0383 & 0.4487 \\
\hline $\mathbf{7}$ & Independent & 0.0276 & 0.4762 \\
\hline $\mathbf{8}$ & Motivated & 0.0409 & 0.5171 \\
\hline $\mathbf{9}$ & Grouchy & 0.0496 & 0.5667 \\
\hline $\mathbf{1 0}$ & Fun & 0.0223 & 0.5889 \\
\hline $\mathbf{1 1}$ & Relieved & 0.0183 & 0.6072 \\
\hline $\mathbf{1 2}$ & Soothing & 0.0219 & 0.6291 \\
\hline $\mathbf{1 3}$ & Annoyed & 0.0156 & 0.6447 \\
\hline $\mathbf{1 4}$ & Loving & 0.0111 & 0.6558 \\
\hline $\mathbf{1 5}$ & Glad & 0.0119 & 0.6677 \\
\hline $\mathbf{1 6}$ & Nostalgic & 0.0148 & 0.6825 \\
\hline $\mathbf{1 7}$ & Good & 0.0104 & 0.6928 \\
\hline $\mathbf{1 8}$ & Aggressive & 0.0114 & 0.7042 \\
\hline $\mathbf{1 9}$ & Attentive & 0.0169 & 0.7211 \\
\hline $\mathbf{2 0}$ & Worried & 0.0117 & 0.7328 \\
\hline $\mathbf{2 1}$ & Secure & 0.0133 & 0.746 \\
\hline $\mathbf{2 2}$ & Alert & 0.0111 & 0.7571 \\
\hline $\mathbf{2 3}$ & Jumpstart & 0.0089 & 0.7661 \\
\hline $\mathbf{2 4}$ & Comfortable & 0.0077 & 0.7737 \\
\hline $\mathbf{2 5}$ & Steady & 0.0087 & 0.7824 \\
\hline $\mathbf{2 6}$ & Balanced & 0.0127 & 0.7951 \\
\hline $\mathbf{2 7}$ & Stable & 0.0126 & 0.8076 \\
\hline $\mathbf{2 8}$ & Collected & 0.0126 & 0.8203 \\
\hline & & & \\
\hline
\end{tabular}




\begin{tabular}{|r|l|r|r|}
\hline \multicolumn{4}{|c|}{ KONA } \\
\hline Step & \multicolumn{1}{|c|}{$\begin{array}{c}\text { Variable } \\
\text { Entered }\end{array}$} & $\begin{array}{c}\text { Partial } \\
\mathbf{R}^{\mathbf{2}}\end{array}$ & $\begin{array}{c}\text { Model } \\
\mathbf{R}^{\mathbf{2}}\end{array}$ \\
\hline $\mathbf{1}$ & Fulfilling & 0.1301 & 0.1301 \\
\hline $\mathbf{2}$ & Affectionate & 0.1066 & 0.2367 \\
\hline $\mathbf{3}$ & Jolted & 0.116 & 0.3527 \\
\hline $\mathbf{4}$ & Annoyed & 0.0643 & 0.417 \\
\hline $\mathbf{5}$ & Happy & 0.0723 & 0.4893 \\
\hline $\mathbf{6}$ & Joyful & 0.0391 & 0.5284 \\
\hline $\mathbf{7}$ & Content & 0.0293 & 0.5577 \\
\hline $\mathbf{8}$ & Active & 0.029 & 0.5868 \\
\hline $\mathbf{9}$ & Tolerated & 0.025 & 0.6118 \\
\hline $\mathbf{1 0}$ & Understanding & 0.0226 & 0.6344 \\
\hline $\mathbf{1 1}$ & Frustrated & 0.023 & 0.6574 \\
\hline $\mathbf{1 2}$ & Pleasant & 0.0174 & 0.6748 \\
\hline $\mathbf{1 3}$ & Warm & 0.0193 & 0.6941 \\
\hline $\mathbf{1 4}$ & Educated & 0.0144 & 0.7084 \\
\hline $\mathbf{1 5}$ & In control & 0.0115 & 0.7199 \\
\hline $\mathbf{1 6}$ & Fun & 0.0159 & 0.7358 \\
\hline $\mathbf{1 7}$ & Worried & 0.0137 & 0.7495 \\
\hline $\mathbf{1 8}$ & Nostalgic & 0.0101 & 0.7596 \\
\hline $\mathbf{1 9}$ & Steady & 0.0091 & 0.7687 \\
\hline $\mathbf{2 0}$ & Sad & 0.0107 & 0.7794 \\
\hline $\mathbf{2 1}$ & Free & 0.0095 & 0.7889 \\
\hline $\mathbf{2 2}$ & Guilty & 0.0126 & 0.8015 \\
\hline $\mathbf{2 3}$ & Motivated & 0.0103 & 0.8118 \\
\hline $\mathbf{2 4}$ & Balanced & 0.0098 & 0.8216 \\
\hline $\mathbf{2 5}$ & Satisfied & 0.0106 & 0.8321 \\
\hline $\mathbf{2 6}$ & Whole & 0.0082 & 0.8403 \\
\hline $\mathbf{2 7}$ & Comfortable & 0.0089 & 0.8493 \\
\hline $\mathbf{2 8}$ & Merry & 0.0104 & 0.8596 \\
\hline $\mathbf{2 9}$ & Soothing & 0.012 & 0.8716 \\
\hline $\mathbf{3 0}$ & Good & 0.0072 & 0.8788 \\
\hline $\mathbf{3 1}$ & Off balance & 0.0073 & 0.8861 \\
\hline $\mathbf{3 2}$ & Productive & 0.0055 & 0.8916 \\
\hline $\mathbf{3 3}$ & Clear minded & 0.0072 & 0.8988 \\
\hline $\mathbf{3 4}$ & Bored & 0.0082 & 0.907 \\
\hline & & & \\
\hline
\end{tabular}

\begin{tabular}{|r|l|r|r|}
\hline \multicolumn{4}{|c|}{ NEWMAN } \\
\hline Step & $\begin{array}{c}\text { Variable } \\
\text { Entered }\end{array}$ & $\begin{array}{c}\text { Partial } \\
\mathbf{R}^{\mathbf{2}}\end{array}$ & $\begin{array}{c}\text { Model } \\
\mathbf{R}^{\mathbf{2}}\end{array}$ \\
\hline $\mathbf{1}$ & Content & 0.2702 & 0.2702 \\
\hline $\mathbf{2}$ & Disappointed & 0.1033 & 0.3735 \\
\hline $\mathbf{3}$ & Boosted & 0.0497 & 0.4232 \\
\hline $\mathbf{4}$ & Mild & 0.0341 & 0.4572 \\
\hline $\mathbf{5}$ & Eager & 0.0328 & 0.4901 \\
\hline $\mathbf{6}$ & Independent & 0.0235 & 0.5136 \\
\hline $\mathbf{7}$ & Home & 0.0244 & 0.5379 \\
\hline $\mathbf{8}$ & Quiet & 0.0226 & 0.5605 \\
\hline $\mathbf{9}$ & Alert & 0.0172 & 0.5778 \\
\hline $\mathbf{1 0}$ & Bored & 0.0157 & 0.5935 \\
\hline $\mathbf{1 1}$ & Balanced & 0.0166 & 0.6101 \\
\hline $\mathbf{1 2}$ & Understanding & 0.02 & 0.6301 \\
\hline $\mathbf{1 3}$ & Productive & 0.0193 & 0.6494 \\
\hline $\mathbf{1 4}$ & Disgusted & 0.0164 & 0.6658 \\
\hline $\mathbf{1 5}$ & Comfortable & 0.0193 & 0.6851 \\
\hline $\mathbf{1 6}$ & Pleasant & 0.0165 & 0.7016 \\
\hline $\mathbf{1 7}$ & Free & 0.0174 & 0.719 \\
\hline $\mathbf{1 8}$ & Active & 0.0149 & 0.7339 \\
\hline $\mathbf{1 9}$ & Motivated & 0.0158 & 0.7497 \\
\hline $\mathbf{2 0}$ & Energetic & 0.0132 & 0.7629 \\
\hline $\mathbf{2 1}$ & Relax & 0.0093 & 0.7722 \\
\hline $\mathbf{2 2}$ & Fun & 0.0098 & 0.7819 \\
\hline $\mathbf{2 3}$ & Excited & 0.0085 & 0.7904 \\
\hline $\mathbf{2 4}$ & Wild & 0.0083 & 0.7987 \\
\hline $\mathbf{2 5}$ & Ready & 0.013 & 0.8116 \\
\hline $\mathbf{2 6}$ & Warm & 0.0111 & 0.8227 \\
\hline $\mathbf{2 7}$ & Good & 0.0082 & 0.8309 \\
\hline $\mathbf{2 8}$ & Tender & 0.0087 & 0.8397 \\
\hline $\mathbf{2 9}$ & Off balance & 0.0072 & 0.8469 \\
\hline $\mathbf{3 0}$ & Merry & 0.0067 & 0.8536 \\
\hline $\mathbf{3 1}$ & Joyful & 0.0084 & 0.862 \\
\hline $\mathbf{3 2}$ & Special & 0.01 & 0.872 \\
\hline $\mathbf{3 3}$ & Soothing & 0.0137 & 0.8857 \\
\hline $\mathbf{3 4}$ & Daring & 0.8945 \\
\hline $\mathbf{3 5}$ & Guilty & 0.9052 \\
\hline $\mathbf{3 6}$ & Educated & 0.9151 \\
\hline & & \\
\hline
\end{tabular}

